\title{
THE WHITEHEAD TRANSFER HOMOMORPHISM FOR ORIENTED $S^{1}$-BUNDLES
}

\section{ROBERT OLIVER}

Consider the following problem.

Question. Given an oriented circle fiber bundle $S^{1} \rightarrow E \rightarrow B$ and a homotopy equivalence $\varphi: B^{\prime} \rightarrow B$, where $E, B$, and $B^{\prime}$ atl are finite complexes; is the pullback homotopy equivalence $\tilde{\varphi}: \varphi^{*}(E) \rightarrow E$ always a simple homotopy equivalence? If not, what can one say about the torsion of $\tilde{\varphi}$ ?

This question arose naturally from the theorem that $\operatorname{Id}_{S^{1}} \times \varphi$ is a simple homotopy equivalence whenever $\varphi$ is a homotopy equivalence of finite complexes [13, Corollary 1.4]. If one works with unoriented $S^{1}$-bundles, it is easy to find examples where $\tau(\tilde{\varphi}) \neq 1$, using, e.g., [2, Corollary C]. The main result here is that examples with $\tau(\tilde{\varphi}) \neq 1$ also occur for oriented $S^{1}$-bundles. Proving the existence of such examples is surprisingly complicated, and provides good test of the current machinery for describing Whitehead groups of finite groups.

The above problem was studied by Munkholm and Pedersen in [16]. They showed that there is a well defined "transfer" map

$$
f^{\#}: \mathrm{Wh}\left(\pi_{1}(B)\right) \rightarrow \mathrm{Wh}\left(\pi_{1}(E)\right)
$$

for any bundle $S^{1} \rightarrow E \rightarrow B$ (not necessarily oriented), depending only on the fundamental group sequence $Z \rightarrow \pi_{1} E \rightarrow \pi_{1} B$, and such that for any homotopy equivalence $\varphi: B^{\prime} \rightarrow B$,

$$
\tau\left(\tilde{\varphi}: \varphi^{*}(E) \rightarrow E\right)=f^{\#}(\tau(\varphi)) .
$$

Conversely, any exact sequence $\mathrm{Z} \stackrel{i}{\longrightarrow} \widetilde{G} \stackrel{\beta}{\longrightarrow} G \rightarrow 1$ of finitely presented groups with $i(Z) \subseteq Z(\widetilde{G})$ can be realized as the fundamental group sequence of some oriented bundle $S^{1} \rightarrow E \rightarrow B$ of finite complexes (see [29, Proposition 11.4 and the following remark]). Such a sequence is described by the pair $(\beta, i(1))$. In other words, given any surjection $\beta: \widetilde{G} \rightarrow G$ of groups such that $\operatorname{Ker}(\beta)$ is cyclic and central in $\widetilde{G}$, and given any generator $z \in \operatorname{Ker}(\beta)$, there is an induced homomorphism

Received June 1, 1984; in revised form May 2, 1985. 


$$
\beta_{z}^{\#}: \mathrm{Wh}(G) \rightarrow \mathrm{Wh}(\tilde{G})
$$

such that $\beta_{z}^{\#}=f^{\#}$ for any oriented bundle $S^{1} \rightarrow E \rightarrow B$ realizing $\beta$ and $z$. This map was described algebraically in [16], and the authors there also listed many cases, when it is trivial. For example, they showed that $\beta_{z}^{\#}=1$ if $\tilde{G}$ is infinite and $G$ is finite, or if $\tilde{G}$ is finite abelian, or more generally if $\widetilde{G}$ is finite and $\operatorname{Ker}(\beta) \cap[\tilde{G}, \tilde{G}]=1$.

Since Wh $(G)$ is so poorly understood for infinite $G$, the natural place to look for examples of $\beta_{z}^{\#} \neq 1$ is when $\tilde{G}$ and $G$ are both finite. By [16, Proposition 6.2],

$$
\operatorname{Im}\left(\dot{\beta}_{z}^{*}\right) \subseteq \mathrm{Cl}_{1}(\mathrm{Z} \tilde{G})=\operatorname{Ker}\left[K_{1}(\mathrm{Z} \tilde{G}) \rightarrow K_{1}(\mathrm{Q} \tilde{G}) \times \prod_{p} K_{1}\left(\hat{Z}_{p} \tilde{G}\right)\right]
$$

in this case. This is the first indication of difficulties in detecting elements in $\operatorname{Im}\left(\beta_{z}^{\#}\right)$ : concrete calculations in $\mathrm{Cl}_{1}(Z \widetilde{G})$ involve almost automatically calculations with $K_{2}\left(\hat{Q}_{p} \tilde{G}\right)$ and $K_{2}\left(\hat{Z}_{p} \tilde{G}\right)$ for primes $p|| \widetilde{G} \mid$. The second indication is that $\beta_{z}^{*}(\mathrm{Wh}(G)) / \beta_{z}^{\#}\left(S K_{1}(Z G)\right)$ has exponent at most 2 (Proposition 1.3) - in fact, as will be seen in Theorem 4.11, $\operatorname{Im}\left(\beta_{z}^{\#}\right)=\beta_{z}^{\#}\left(S K_{1}(Z G)\right)$, if $G$ has normal 2-Sylow subgroup, and we know of no example where the groups differ. Finally, $\mathrm{Cl}_{1}(\mathrm{ZG}) \subseteq \operatorname{Ker}\left(\beta_{z}^{\#}\right)$, and

$$
S K_{1}(\mathrm{Z} G) / \mathrm{Cl}_{1}(\mathrm{Z} G) \cong \prod_{p} S K_{1}\left(\hat{\mathrm{Z}}_{p} G\right)
$$

is non-zero only when $G$ is fairly complicated - sufficiently complicated that describing $\mathrm{Cl}_{1}(\mathrm{ZG})$ becomes (at best) a rather messy combinatorial problem.

Sections 1 and 2 contain background material: Section 1 is mostly a recapitulation of results from [16], including the algebraic definition of the $\beta_{z}^{\#}$; while Section 2 is a collection of general results which are needed later for calculations in localization sequences. Examples with $\beta_{z}^{\#} \neq 1$ are constructed in Section 3, showing, in fact, that $p$-torsion can occur in $\operatorname{Im}\left(\beta_{z}^{\#}\right)$ for any prime $p$.

The $\beta_{z}^{\#}$ are further analyzed in Section 4, in an attempt to explain why such examples need be so complicated. The main general results there, for surjections $\beta: \widetilde{G} \rightarrow G$ of finite groups with $\operatorname{Ker}(\beta)$ central and cyclic are:

(a) $\beta^{\#}=\beta_{z}^{\#}$ is independent of the choice of generator $z \in \operatorname{Ker}(\beta)$ (this is not at all obvious from the geometry).

(b) for any prime $p$, if $K=\{g \in \operatorname{Ker}(\beta): p \nmid|g|\}$, then

$\operatorname{Ker}\left[\beta_{(p)}^{*}: \operatorname{Wh}(G) \rightarrow \mathrm{Cl}_{1}(\mathrm{Z} \tilde{G})_{(p)}\right]=\operatorname{Ker}\left[(\beta / K)^{*}: \operatorname{Wh}(G) \rightarrow \mathrm{Cl}_{1}(\mathrm{Z}[\tilde{G} / K])\right]$ 
(see Theorem 4.10 for more detail). The problem of describing $\beta^{\#}$ is thus reduced to the case where $\operatorname{Ker}(\beta)$ is a $p$-group.

(c) if $\operatorname{Ker}(\beta)$ is a $p$-group for some $p$, then $\beta^{\#}$ factors as a composite

$$
\beta^{\#}: \mathrm{Wh}(G) \stackrel{\Omega_{\beta}}{\longrightarrow} W_{\beta} / W_{\beta}^{\prime} \stackrel{\Theta_{\beta}}{\longrightarrow} \mathrm{Cl}_{1}(\mathrm{Z} \tilde{G}),
$$

where $W_{\beta}^{\prime} \subseteq W_{\beta}$ are defined explicitly in Proposition 4.8.

The problem of describing $\beta^{\#}$ is thus split into two independent problems: one involving $\mathrm{Wh}(G)$, and the other involving $\mathrm{Cl}_{1}(\mathrm{Z} \widetilde{G})$. For more detail, see Theorem 4.9 and the following discussion. Some general consequences of the above decomposition are listed in Theorem 4.11.

In what follows, we work, for convenience, with $K_{1}(Z G)$ rather than Wh $(G)$ : that the $\pm g$ lie in $\operatorname{Ker}\left(\beta_{z}^{\#}\right)$ is immediate from the algebraic definition of $\beta_{z}^{\#}$. By $\zeta_{n}$, for $n \geqq 1$, will always be meant the primitive root of unity $e^{2 \pi i / n}$ (so that $\left(\zeta_{n}\right)^{n / m}=\zeta_{m}$ for $m \mid n$ ). We use $C_{n}$ to denote a (multiplicative) cyclic group of order $n$. For any $G$ and any $g \in G$, $N(g)=N_{G}(g)$, and $C(g)=C_{G}(g)$ denote the normalizer and centralizer, respectively. And $K_{2}^{c}(R)$, for any $Q_{p}$-algebra of $\hat{Z}_{p}$-order $R$, denotes the continuous $K_{2}$ (as defined in Section 2).

Finally, my thanks to Erik Kjær Pedersen and Hans Munkholm for bringing this problem to my attention, and for several discussions about it.

1.

It will be useful to have the algebraic "transfer" maps defined in greater generality, as follows. For convenience in notation, $\left(a_{i j}\right)$ and $\left[a_{i j}\right]$, respectively, will be used to denote a matrix over a ring $R$ and its class in $K_{1}(R)$.

THeOREM 1.1. Let $R$ be a ring, and let $\eta \in R$ be a fixed central element. Then there is a well defined homomorphism

$$
\eta^{\#}: K_{1}(R / \eta R) \rightarrow K_{1}(R)
$$

such that, for any $\bar{A} \in \mathrm{GL}_{n}(R / \eta R)$, any lifting $A \in M_{n}(R)$ of $\bar{A}$, and any $B$, $X \in M_{n}(R)$ such that $A B-\eta X=I$,

$$
\eta^{\#}([\bar{A}])=\left[\begin{array}{cc}
A & X \\
\eta & B
\end{array}\right] .
$$

Furthermore,

(i) if $\mathrm{pr}_{\eta}: R \rightarrow R / \eta R$ denotes the projection, then

$$
\operatorname{Im}\left(K_{1}\left(\operatorname{pr}_{\eta}\right)\right) \subseteq \operatorname{Ker}\left(\eta^{\#}\right) \text { and } \operatorname{Im}\left(\eta^{\#}\right) \subseteq \operatorname{Ker}\left(K_{1}\left(\operatorname{pr}_{\eta}\right)\right) \text {. }
$$


(ii) For any central unit $v$ in $R,(\eta v)^{\#}=\eta^{\#}$.

(iii) If $f: R \rightarrow S$ is any ring homomorphism such that $f(\eta)$ is central in $S$, then the following square commutes:

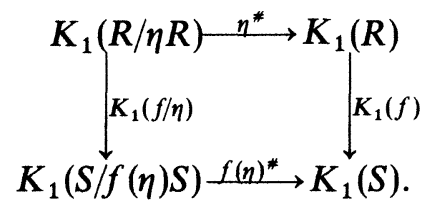

Proof. Except for (ii), this is included in [16, Theorem 1.1]. And (ii) follows upon noting that for any $A, B, X$ as above,

$$
\left[\begin{array}{ll}
A & X \\
\eta & B
\end{array}\right]=\left[\left(\begin{array}{ll}
I & 0 \\
0 & v
\end{array}\right)\left(\begin{array}{ll}
A & X \\
\eta & B
\end{array}\right)\left(\begin{array}{cc}
I & 0 \\
0 & v^{-1}
\end{array}\right)\right]=\left[\begin{array}{cc}
A & v^{-1} X \\
\eta v & B
\end{array}\right] \in K_{1}(R) .
$$

Now, if $\beta: G \rightarrow G$ is any surjection of groups whose kernel is cyclic and central in $\widetilde{G}$, and if $z \in \operatorname{Ker}(\beta)$ is any generator, we set

$$
\beta_{z}^{\#}=(1-z)^{\#}=(z-1)^{\#}=\left(1-z^{-1}\right)^{\#}: K_{1}(Z G) \rightarrow K_{1}(Z \tilde{G}) .
$$

Note that these are all the same by (ii) above. By $[16$, Theorem $\mathrm{A}], \beta_{z}^{\#}$ is the Whitehead transfer homomorphism for any oriented $S^{1}$-bundle whose fundamental group exact sequence is $Z \stackrel{i}{\longrightarrow} \widetilde{G} \stackrel{\beta}{\longrightarrow} G$, where $i(1)=z$.

If $G$ is any finite group, we define

$$
\mathrm{Cl}_{1}(\mathrm{Z} G)=\operatorname{Ker}\left[K_{1}(\mathrm{Z} G) \rightarrow K_{1}(\mathrm{Q} G) \times \prod_{p} K_{1}\left(\hat{Z}_{p} G\right)\right]
$$

(the product being taken over all primes $p$ ).

Proposition 1.2. Let $\beta: G \rightarrow G$ be any surjection of finite groups such that $\operatorname{Ker}(\beta)$ is cyclic and central in $\tilde{G}$. Then, for any generator $z$ of $\operatorname{Ker}(\beta)$,

$$
\operatorname{Im}\left(\beta_{z}^{\#}\right) \subseteq \mathrm{Cl}_{1}(\mathrm{Z} \tilde{G}) \text { and } \mathrm{Cl}_{1}(\mathrm{ZG}) \subseteq \operatorname{Ker}\left(\beta_{z}^{\#}\right) \text {. }
$$

Proof. The induced maps

$$
K_{1}(Q \beta): K_{1}(Q \tilde{G}) \rightarrow K_{1}(Q G) \text { and } K_{1}\left(\hat{Z}_{p} \beta\right): K_{1}\left(\hat{Z}_{p} \tilde{G}\right) \rightarrow K_{1}\left(\hat{Z}_{p} G\right)
$$

are surjective by [8, Corollary III.2.9]: $Q \beta$ and the $\hat{Z}_{p} \beta$ are all surjections of semilocal rings. So by Theorem $1.1(i)$, the transfer maps $(1-z)^{\#}$ on the rational and $p$-adic group rings are zero. It follows that

$$
\operatorname{Im}\left(\beta_{z}^{*}\right) \subseteq \operatorname{Ker}\left[K_{1}(\mathrm{Z} \tilde{G}) \rightarrow K_{1}(\mathrm{Q} \tilde{G}) \times \prod_{p} K_{1}\left(Z_{p} \tilde{G}\right)\right]=\mathrm{Cl}_{1}(\mathrm{Z} \tilde{G}) .
$$

By [17, Lemma 1], the induced map from $\mathrm{Cl}_{1}(\mathrm{Z} \tilde{G})$ to $\mathrm{Cl}_{1}(\mathrm{ZG})$ is onto. Together with Theorem 1.1, this implies that 


$$
\operatorname{Ker}\left(\beta_{z}^{\#}\right) \supseteq \operatorname{Im}\left(K_{1}(\beta)\right) \supseteq \mathrm{Cl}_{1}(\mathrm{Z} G) .
$$

The relationship between $\operatorname{Im}\left(\beta_{z}^{\#}\right)$ and $\beta_{z}^{*}\left(S K_{1}(Z G)\right)$, and the reason why it is easier to detect elements in $\beta_{z}^{\#}\left(S K_{1}(Z G)\right)$ than in $\operatorname{Im}\left(\beta_{z}^{\#}\right) / \beta_{z}^{\#}\left(S K_{1}(Z G)\right)$ will be considered in detail in Section 4 . But the first indication of the important role played by $S K_{1}(Z G)$ comes from the next proposition.

Proposition 1.3. Let $\beta: \widetilde{G} \rightarrow G$ and $z \in \operatorname{Ker}(\beta)$ be as in Proposition 1.2. Then

$$
\exp \left[\beta_{z}^{\#}\left(K_{1}(Z G) / \beta_{z}^{\#}\left(S K_{1}(Z G)\right)\right] \leqq 2 .\right.
$$

Proof. For any $x=\sum \lambda_{i} g_{i} \in Z G$, set $\bar{x}=\sum \lambda_{i} g_{i}^{-1}$. For any $M$ $=\left(x_{i j}\right) \in M_{n}(Z G)$, set $\bar{M}=\left(\bar{x}_{i i}\right) ;$ i.e., the conjugate transpose matrix. Then $(M \rightarrow \bar{M})$ induces an involution of $K_{1}(Z \bar{G})$; and similarly for $K_{1}(Z G)$.

Fix $u=[M] \in K_{1}(Z G)$, where $M \in \mathrm{GL}_{n}(Z G)$, lift $M$ and $M^{-1}$ to $A, B \in M_{n}(Z G)$, and choose $X \in M_{n}(Z G)$ such that $A B-(1-z) X=I$. Then

$$
\begin{aligned}
\overline{\beta_{z}^{\#}(u)}=\left[\begin{array}{cc}
\bar{A} & 1-z^{-1} \\
\bar{X} & \bar{B}
\end{array}\right] & =\left[\left(\begin{array}{cc}
0 & I \\
-z I & 0
\end{array}\right)\left(\begin{array}{cc}
\bar{A} & 1-z^{-1} \\
\bar{X} & \bar{B}
\end{array}\right)\left(\begin{array}{cc}
0 & -z^{-1} I \\
I & 0
\end{array}\right)\right] \\
& =\left[\begin{array}{cc}
\bar{B} & -z^{-1} \bar{X} \\
1-z & \bar{A}
\end{array}\right]=\beta_{z}^{\#}\left(\bar{u}^{-1}\right) .
\end{aligned}
$$

On the other hand, the involution fixes $K_{1}(\mathrm{Z} G) / S K_{1}(\mathrm{Z} G) \times( \pm G)$ by $[30$, Proposition 10.1]; and it fixes $\mathrm{Cl}_{1}(\mathrm{ZG})$ by [4, Theorem 2] (the result is stated in [4] only for odd order nilpotent groups; but holds in general by the same argument, and the later results of Bak and Rehmann discussed in Theorem 2.2 below). Since $\beta_{z}^{\#}$ is negative equivariant with respect to the involution, and the involution fixes the domain and range of

$$
\left(\beta_{z}^{\#}\right)^{\prime}: K_{1}(Z G) / S K_{1}(Z G) \times( \pm G) \rightarrow \mathrm{Cl}_{1}(\mathrm{Z} G) / \beta_{z}^{\#}\left(S K_{1}(\mathrm{Z} G)\right),
$$

the image has exponent at most 2.

The last result in this section also helps to suggest the direction in which one must go to find examples with $\beta_{z}^{\#} \neq 1$.

Proposition 1.4. Let $\beta: \widetilde{G} \rightarrow G$ and $z \in \operatorname{Ker}(\beta)$ be as in Proposition 1.2. Then $\operatorname{Im}\left(\beta_{z}^{\#}\right)$ is finite, and has torsion only for primes dividing the order of $\operatorname{Ker}(\beta) \cap[\tilde{G}, \tilde{G}]$.

Proof. If $\operatorname{Ker}(\beta) \cap[\widetilde{G}, \widetilde{G}]=1$, then $\beta$ is the reduction of some central extension $1 \rightarrow Z \rightarrow \hat{G} \rightarrow G \rightarrow 1$. So $\beta_{z}^{\#}=1$ in this case by [16, Proposition $6.1]$, and naturality.

The image of $\beta_{z}^{\#}$ is finite since $\mathrm{Cl}_{1}(\mathrm{Z} \tilde{G})$ is. It remains to show, for any 
prime $p$, that $\operatorname{Im}\left(\beta_{z}^{\#}\right)$ is $p$-torsion free if $\operatorname{Ker}(\beta) \cap[\widetilde{G}, \widetilde{G}]$ is. It suffices to prove this when $G$ is $p$-hyperelementary: $K_{1}(Z G)_{(p)}$ is generated by $p$ hyperelementary induction otherwise [14]. But if $G$ is $p$-hyperelementary and $\operatorname{Ker}(\beta)$ is central and $p$-torsion free, then $\operatorname{Ker}(\beta) \cap[\widetilde{G}, \widetilde{G}]=1$; and so $\beta_{z}^{\#}=1$.

This last result will be sharpened in Section 4.

\section{2.}

A fair amount of machinery is now needed before further results on the $\beta_{z}^{\#}$ can be obtained. These background results will be collected here, and applied in Sections 3 and 4.

If $\mathfrak{U}$ is a $\hat{Z}_{p}$-order in a finite dimensional semisimple $\hat{Q}_{p}$-algebra $A$ (for some prime $p$ ), define

$$
K_{2}^{c}(\mathfrak{H})=\lim _{\bar{n}} \operatorname{Coker}\left[K_{2}\left(\mathfrak{H}, p^{n} \mathfrak{U}\right) \rightarrow K_{2}(\mathfrak{U})\right]
$$

and, if $\mathfrak{M} \subseteq A$ is any maximal $\hat{Z}_{p}$-order,

$$
K_{2}^{c}(A)=\lim _{\bar{n}} \operatorname{Coker}\left[K_{2}\left(\mathfrak{M}, p^{n} \mathfrak{M}\right) \rightarrow K_{2}(A)\right] .
$$

These groups turn out to be the most convenient to use when making calculations in $K_{2}$ of $p$-adic orders and algebras. That $K_{2}^{c}(A)$ is independent of the choice of $\mathfrak{M}$ follows easily from the fact that any two maximal orders in $A$ are conjugate in $A$ (see, e.g., [24, Theorem 17.3]).

If $\mathfrak{A}$ is a Z-order in a finite dimensional semisimple Q-algebra $A$, then by definition

$$
\begin{aligned}
& S K_{1}(\mathfrak{U})=\operatorname{Ker}\left[K_{1}(\mathfrak{H}) \rightarrow K_{1}(A)\right], \\
& S K_{1}\left(\hat{\mathfrak{U}}_{p}\right)=\operatorname{Ker}\left[K_{1}\left(\hat{\mathfrak{U}}_{p}\right) \rightarrow K_{1}\left(\hat{A}_{p}\right)\right]
\end{aligned}
$$

for any prime $p\left(\hat{\mathfrak{U}}_{p}=\hat{Z}_{p} \otimes_{\mathrm{Z}} \mathfrak{U}\right.$ and $\left.\hat{A}_{p}=\hat{Q}_{p} \otimes_{\mathrm{Q}} A\right)$; and

$$
\mathrm{Cl}_{1}(\mathfrak{U})=\operatorname{Ker}\left[S K_{1}(\mathfrak{U}) \rightarrow \prod_{p} S K_{1}\left(\hat{\mathfrak{A}}_{p}\right)\right] .
$$

There are several itypes of localization sequences which can be used to describe and make calculations in $S K_{1}(\mathfrak{U})$ and $\mathrm{Cl}_{1}(\mathfrak{U})$ (see, e.g., [5] or [11]); the following one will suffice for our purpose here.

THEOREM 2.1. Let $\mathfrak{A}$ be any Z-order in a finite dimensional semisimple Qalgebra $A$, and let $\mathfrak{M} \subseteq A$ be a maximal order containing $\mathfrak{U}$. Fix a prime $p$. Then there are exact sequences 
(1) $\quad K_{2}^{c}\left(\hat{\mathfrak{U}}_{p}\right) \rightarrow \operatorname{Coker}\left[K_{2}(\mathfrak{M}[1 / p])_{(p)} \rightarrow K_{2}^{c}\left(\hat{A}_{p}\right)_{(p)}\right] \stackrel{\partial}{\rightarrow} \mathrm{Cl}_{1}(\mathfrak{H})_{(p)} \rightarrow 1 \quad$. and Furthermore,

$$
1 \rightarrow \mathrm{Cl}_{1}(\mathfrak{A}) \rightarrow S K_{1}(\mathfrak{A}) \rightarrow \prod_{\substack{q \\ \text { prime }}} S K_{1}\left(\hat{\mathfrak{U}}_{q}\right) \rightarrow 1
$$

$$
K_{2}^{c}\left(\hat{\mathfrak{U}}_{p}\right) \cong \lim _{\hbar} K_{2}\left(\mathfrak{H} / p^{n} \mathfrak{I}\right)
$$

(ii) $\operatorname{Coker}\left[K_{2}(\mathfrak{M}) \rightarrow K_{2}^{c}\left(\hat{\mathfrak{M}}_{p}\right)\right] \cong \operatorname{Coker}\left[K_{2}(\mathfrak{M}[1 / p]) \rightarrow K_{2}^{c}\left(\hat{A}_{p}\right)\right]$.

(iii) Fix $A \in \mathrm{GL}_{n}(\mathfrak{U})$ such that

$$
[A] \in \operatorname{Ker}\left[K_{1}(\mathfrak{U}) \rightarrow K_{1}\left(\hat{\mathfrak{U}}_{p}\right) \oplus K_{1}(\mathfrak{M}[1 / p])\right] \subseteq \mathrm{Cl}_{1}(\mathfrak{U}) .
$$

Lift $A$ to elements $x_{1} \in \operatorname{St}\left(\hat{\mathfrak{Q}}_{p}\right)$ and $x_{2} \in \operatorname{St}(\mathfrak{M}[1 / p])$. Then $x_{1} x_{2}^{-1} \in K_{2}\left(\hat{A}_{p}\right)$, and $[A]=\partial\left(x_{1} x_{2}^{-1}\right)$.

Proof. Step 1. To show (i), first fix some

$$
\left(\left[u_{n}\right]\right)_{n=1}^{\infty} \in \lim _{\hbar} \operatorname{Ker}\left[K_{1}\left(\hat{\mathfrak{U}}_{p}, p^{n} \hat{\mathfrak{U}} \hat{\mathfrak{H}}_{p}\right) \rightarrow K_{1}\left(\hat{\mathfrak{Q}}_{p}\right)\right]
$$

where $u_{n} \in 1+p^{n} \hat{\mathfrak{A}}_{p}$ for all $n$. For any $m>n \geqq 1$,

$$
u_{m} \equiv u_{n}\left(\bmod K_{n}=\operatorname{Ker}\left[\left(1+p^{n} \hat{\mathfrak{U}}_{p}\right) \rightarrow K_{1}\left(\hat{\mathfrak{U}}_{p}, p^{n} \hat{\mathfrak{U}}_{p}\right)\right]\right) \text {. }
$$

The $K_{n}$ are closed in the $p$-adic topology [18, Lemma 1], and so

$$
u_{n} \equiv \lim _{m \rightarrow \infty} u_{m}=1\left(\bmod K_{n}\right) \text {. }
$$

In other words, $\left[u_{n}\right]=1$ for all $n$, and the limit in (3) is trivial. On the other hand, since $K_{2}\left(\mathfrak{U} / p^{n} \mathfrak{U}\right.$ ) is finite for all $n$ (see, e.g., [20, Theorem 2.4]), the exact sequences for the pairs $\left(\hat{\mathfrak{U}}_{p}, p^{n} \hat{\mathfrak{Q}}_{p}^{r}\right)$ induce a short exact sequence

$$
\begin{gathered}
1 \rightarrow \lim _{\bar{n}} \operatorname{Coker}\left[K_{2}\left(\hat{\mathfrak{U}}_{p}, p^{n} \hat{\mathfrak{U}}_{p}\right) \rightarrow K_{2}\left(\hat{\mathfrak{U}}_{p}\right)\right] \rightarrow \lim _{\bar{n}} K_{2}\left(\mathfrak{U} / p^{n} \mathfrak{U}\right) \\
\rightarrow \lim _{\bar{n}} \operatorname{Ker}\left[K_{1}\left(\hat{\mathfrak{U}}_{p}, p^{n} \hat{\mathfrak{U}}_{p}\right) \rightarrow K_{1}\left(\hat{\mathfrak{U}}_{p}\right)\right] \rightarrow 1 ;
\end{gathered}
$$

and (i) follows.

STEP 2. By [12], $\mathrm{Cl}_{1}(\mathfrak{M})=1$. By [23, Theorems 4 and 5], there are exact sequences 
- $1 \rightarrow K_{2}(\mathfrak{M}) \rightarrow K_{2}(\mathfrak{M}[1 / p]) \rightarrow K_{1}\left(\hat{\mathfrak{M}}_{p} / J\right) \rightarrow K_{1}(\mathfrak{M}) \rightarrow K_{1}(\mathfrak{M}[1 / p])$

(4)

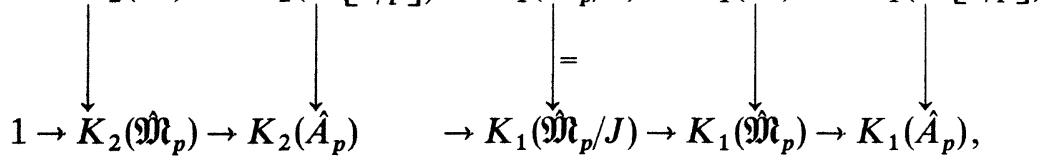

where $J \subseteq \mathfrak{M}_{p}$ is the Jacobson radical. The bottom row is clearly still exact if $K_{2}$ is replaced by $K_{2}^{c}$. Also,

$$
\operatorname{Ker}\left[K_{1}(\mathfrak{M}) \rightarrow K_{1}(\mathfrak{M}[1 / p]) \oplus K_{1}\left(\hat{\mathfrak{M}}_{p}\right)\right] \subseteq \mathrm{Cl}_{1}(\mathfrak{M})=1 .
$$

So the snake lemma applied to (4) induces an isomorphism

(5) $\operatorname{Coker}\left[K_{2}(\mathfrak{M}) \rightarrow K_{2}^{c}\left(\hat{\mathfrak{M}}_{p}\right)\right] \cong \operatorname{Coker}\left[K_{2}(\mathfrak{M}[1 / p]) \rightarrow K_{2}^{c}\left(\hat{A}_{p}\right)\right]$.

By [20, Proposition 1.2], there is an exact sequence

$$
K_{2}^{c}\left(\hat{\mathfrak{U}}_{p}\right) \rightarrow \operatorname{Coker}\left[K_{2}(\mathfrak{M} \overline{\mathfrak{i}}) \rightarrow K_{2}^{c}\left(\hat{\mathfrak{M}}_{p}\right)\right] \stackrel{\partial}{\longrightarrow} \mathrm{Cl}_{1}(\mathfrak{H})_{(p)} \rightarrow 0
$$

(note that $K_{2}^{c}\left(\mathfrak{M}_{p}\right)$ is finite, by [7, Corollary 4.4 and Theorem 4.7]). Sequence (1) now follows upon combining this with (5). The description in (iii) of the boundary map follows from [20, Proposition 1.1]. Finally, the exactness of (2) follows from the localization sequences in [5], [11], or $[20]$ : note that

$$
S K_{1}(\mathfrak{M}) \cong \prod_{q} S K_{1}\left(\hat{\mathfrak{M}}_{q}\right)
$$

by [12]; and that $S K_{1}\left(\hat{\mathfrak{U}}_{q}\right)$ is trivial for almost all $q$ (since $\hat{\mathfrak{A}}_{q}$ is maximal and $\hat{A}_{q}$ a product of matrix algebras over fields for almost all $q$ ).

If $A$ is a finite dimensional semisimple Q-algebra, and $\mathfrak{M} \subseteq A$ is a maximal order, then for any prime $p$ we set

$$
C_{p}(A)=\operatorname{Coker}\left[K_{2}(\mathfrak{M}[1 / p]) \rightarrow K_{2}^{c}\left(\hat{A}_{p}\right)\right] \cong \operatorname{Coker}\left[K_{2}(\mathfrak{M}) \rightarrow K_{2}^{c}\left(\hat{M}_{p}\right)\right]
$$

These groups have been completely described: by Bass, Milnor and Serre [9], when $A$ is a product of matrix algebras over fields; and by Bak and Rehmann ([7] and [6]) in the general case. The description is in terms of norm residue symbols.

If $K$ is any finite extension of $\hat{Q}_{p}$, and $\mu \subseteq K^{*}$ is any group of roots of unity in $K$, then a symbol with value in $\mu$ :

$$
(\cdot, \cdot)_{v}: K^{*} \times K^{*} \rightarrow \mu
$$

is defined (see, e.g., [26, Section XIV.2]). For convenience, we will let

$$
(\cdot, \cdot)_{K}: K^{*} \times K^{*} \rightarrow\left(\mu_{K}\right)_{p}
$$


denote the symbol with values in $\left(\mu_{K}\right)_{p}$, the group of all $p$-power roots of unity in $K$. If $K$ is a number field, then

$$
(\cdot, \cdot)_{K}:\left(\hat{K}_{p}\right)^{*} \times\left(\hat{K}_{p}\right)^{*} \rightarrow\left(\mu_{K}\right)_{p}
$$

will denote the product of the symbols for the various $\hat{K}_{p}$ for prime ideals $p \mid p$. This last does, of course, depend on $p$; but we leave that out to keep the notation from getting too complicated.

Finally, if $B$ is a finite dimensional central simple $K$-algebra, then $\mathrm{nr}_{B / K}: B^{*} \rightarrow K^{*}$ will denote the reduced norm homomorphism (see, e.g., [24, Section 9]). Note that when $B=M_{r}(K)$, then $\mathrm{nr}_{B / K}$ is just the determinant map.

THEOREM 2.2. Let $B$ be a simple algebra which is finite dimensional over its center $K$. For any prime $p,\left(\mu_{K}\right)_{p}$ denotes the group of p-power roots of unity in K.

(i) If $K$ is a finite extension of $\mathrm{Q}_{p}$ for some prime $p$, then there is an isomorphism

$$
\lambda_{B}: K_{2}^{c}(B)_{(p)} \cong\left(\mu_{K}\right)_{p}
$$

such that for any $u \in K^{*}$ and $v \in B^{*}$,

$$
\lambda_{B}(\{u, v\})=\left(u, \mathrm{nr}_{B / K}(v)\right)_{K} .
$$

(ii) Assume $K$ is a finite extension of $\mathrm{Q}$, and fix a prime $p$. If, for some real valuation $K \subseteq \mathrm{R}, \mathrm{R} \otimes_{K} B$ is a matrix algebra over $\mathrm{R}$, then $C_{p}(B)=1$. Otherwise, there is an isomorphism

$$
\lambda_{B}: C_{p}(B) \stackrel{\cong}{\leftrightarrows}\left(\mu_{K}\right)_{p}
$$

such that, for any $u \in\left(\hat{K}_{p}\right)^{*}$ and $v \in\left(\hat{B}_{p}\right)^{*}$,

$$
\lambda_{B}(\{u, v\})=\left(u, \operatorname{nr}_{B / K}(v)\right)_{K},\left(\{u, v\} \in C_{p}(B)=K_{2}^{c}\left(\hat{B}_{p}\right) / K_{2}(\mathfrak{M}[1 / p])\right) .
$$

PRoof. We refer to the results in [7] and [6]. The authors only work there with division algebras; but the results are easily extended to matrix algebras over division algebras.

(i) This follows from [7, Theorem 4.7] and [6, Corollary].

(ii) By [7, Theorem 5.2] and [6],

$$
\text { Coker }\left[K_{2}(B) \rightarrow \sum_{q} K_{2}^{c}\left(\hat{B}_{q}\right)\right] \cong \begin{cases}1 & \text { if some } K \rightarrow \mathrm{R} \text { splits } B \\ \mu_{K} & \text { otherwise. }\end{cases}
$$

Here, the sum is taken over all primes $q$, and the isomorphism in the second case is given by norm residue symbols and reduced norms. 
Let $\mathfrak{M} \subseteq B$ be any maximal order. For any prime $q$,

$$
K_{2}^{c}\left(\hat{\mathfrak{M}}_{q}\right) \cong \underset{\leftarrow}{\lim } K_{2}\left(\mathfrak{M} / q^{n} \mathfrak{M}\right)
$$

is a pro-q-group [20, Theorem 2.4]. So the localization exact sequences of $\left[23\right.$, Theorems 4 and 5], applied to the pairs $\mathfrak{M}[1 / p] \subseteq B, \hat{B}_{p} \subseteq \hat{B}_{p}$, and $\mathfrak{M}_{q} \subseteq \hat{B}_{q}(q \neq p)$ take $p$-locally the form:

$$
\begin{aligned}
& 1 \rightarrow K_{2}(\mathfrak{M}[1 / p])_{(p)} \rightarrow K_{2}(B)_{(p)} \rightarrow \sum_{q \neq p} K_{2}\left(\hat{M}_{q} / J\right)_{(p)} \rightarrow S K_{1}(\mathfrak{M})_{(p)} \rightarrow 1 \\
& 1 \rightarrow K_{2}^{c}\left(\hat{B}_{p}\right)_{(p)} \rightarrow \quad \sum_{q} K_{2}^{c}\left(\hat{B}_{q}\right)_{(p)} \rightarrow \sum_{q \neq p} K_{2}\left(\hat{\mathfrak{M}}_{q} / J\right)_{(p)} \rightarrow \sum_{q \neq p} S K_{1}\left(\hat{\mathfrak{M}}_{q}\right)_{(p)} \rightarrow 1 .
\end{aligned}
$$

Here, $J$ denotes Jacobson radical. By [12],

$$
S K_{1}(\mathfrak{M}) \cong \sum_{q} S K_{1}\left(\hat{\mathfrak{M}}_{q}\right) \text { and } S K_{1}\left(\hat{\mathfrak{M}}_{p}\right)_{(p)}=1 .
$$

It follows that

$$
C_{p}(B)=\operatorname{Coker}\left[K_{2}(\mathfrak{M}[1 / p]) \rightarrow K_{2}^{c}\left(\hat{B}_{p}\right)\right]_{(p)} \cong \operatorname{Coker}[B) \rightarrow \sum_{q} K_{2}^{c}\left(\hat{B}_{q}\right)_{(p)},
$$

and (ii) now follows from (1) above.

In order to apply Theorem 2.2 in concrete calculations, some formulas and relations for norm residue symbols are needed. The following two theorems will suffice for our purposes here.

THEOREM 2.3. Fix some prime power $p^{n}(n \geqq 1)$, set $\zeta=\zeta_{p^{n}}$, and let $K=\hat{Q}_{p}(\zeta)$. Let $\operatorname{Tr}: K \rightarrow \hat{Q}_{p}$ be the trace map. Then

(i) for any $u \in 1+(1-\zeta) \hat{Z}_{p}[\zeta],(\zeta, u)_{K}=\zeta^{R}$, where

$$
\begin{array}{ll}
R=\frac{1}{p^{n}} \operatorname{Tr}(\log (u)) & \text { if } p \text { is odd } \\
R=\left(1+2^{n-1}\right) \cdot \frac{1}{2^{n}} \operatorname{Tr}(\log (u)) & \text { if } p=2 \text { and } n \geqq 2,
\end{array}
$$

(ii) for any $u \in 1+(1-\zeta) \hat{Z}_{p}[\zeta],(1-\zeta, u)_{K}=\zeta^{S}$, where

$$
S=\frac{-1}{p^{n}} \operatorname{Tr}\left(\frac{\zeta}{1-\zeta} \log (u)\right),
$$

(iii) if $u, u^{\prime} \in 1+(1-\zeta) \vec{Z}_{p}[\zeta]$ are such that $\log (u)=\log \left(u^{\prime}\right)$, then for any $i \notin p^{n} \mathrm{Z}$ (that is, $\left.\zeta^{i} \neq 1\right)$ : 


$$
\left(1-\zeta^{i}, u\right)_{K}=\left(1-\zeta^{i}, u^{\prime}\right)_{K}
$$

Proof. These are due to Artin and Hasse [3]. Note that (iii) follows immediately from (ii) if $i=1$, and hence (by symmetry) whenever $p \nmid i$. It holds in general since for any $k \leqq n$ and any $i$ prime to $p$ :

$$
1-\zeta^{i p^{k}}=\prod_{j=0}^{p^{k}-1}\left(1-\zeta^{i+j p^{n-k}}\right)
$$

THeOREM 2.4. Fix a prime $p$, fix finite extensions $K \supseteqq F \supseteqq \hat{Q}_{p}$, and let $\hat{\mu} \subseteq K^{*}$ and $\mu \subseteq F^{*} \cap \hat{\mu}$ be groups of p-power roots of unity. Then for any $u \in F^{*}$ and $v \in K^{*}$,

$$
\left(u, N_{\mathbf{K} / F}(v)\right)_{\mu}=\left((u, v)_{\hat{\mu}}\right)^{[\hat{\mu}: \mu]} .
$$

Proof. Set $p^{l}=|\hat{\mu}|$ and $p^{k}=|\mu|$. Fix some extension $K(\alpha) / K$ such that $\alpha^{p^{l}}=u$. The diagram

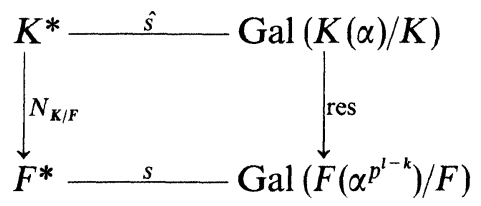

commutes by [26, Section XI.3], where $\hat{s}$ and $s$ are the reciprocity maps, and res is induced by restriction. By [26, Proposition XIV.6],

$$
\begin{aligned}
\left(u, N_{K / F}(v)\right)_{\mu} & =s\left(N_{K / F}(v)\right)\left(\alpha^{p^{l-k}}\right) / \alpha^{p^{l-k}} \\
& =[\hat{s}(v)(\alpha) / \alpha]^{p^{l-k}}=\left((u, v)_{\hat{\mu}}\right)^{p^{l-k}} .
\end{aligned}
$$

Next some means is needed for lifting an element in the image of a transfer map from $\mathrm{Cl}_{1}(\mathrm{Z} \tilde{G})$ to $C_{p}(\mathrm{Q} \tilde{G})$.

LEMMA 2.5. Let $R$ be any ring, and let $\left(\begin{array}{ll}a & b \\ c & d\end{array}\right) \in M_{2}(R)$ be such that ac $=c a$, and $a d-c b=1$.

(i) If $a \in R^{*}$, then

$$
\left(\begin{array}{ll}
a & b \\
c & d
\end{array}\right)=\left(\begin{array}{cc}
1 & 0 \\
c a^{-1} & 1
\end{array}\right)\left(\begin{array}{cc}
a & 0 \\
0 & a^{-1}
\end{array}\right)\left(\begin{array}{cc}
1 & a^{-1} b \\
0 & 1
\end{array}\right) \in E_{2}(R) .
$$

(ii) If $c \in R^{*}$, then

$$
\left(\begin{array}{ll}
a & b \\
c & d
\end{array}\right)=\left(\begin{array}{cc}
1 & a c^{-1} \\
0 & 1
\end{array}\right)\left(\begin{array}{cc}
0 & -c^{-1} \\
c & 0
\end{array}\right)\left(\begin{array}{cc}
1 & c^{-1} d \\
0 & 1
\end{array}\right) \in E_{2}(R) .
$$

(iii) If $a, c \in R^{*}$, then

$$
\left(x_{21}^{c a^{-1}} h_{21}(a)^{-1} x_{12}^{a^{-1} b}\right)\left(x_{12}^{a c^{-1}} w_{21}(c) x_{12}^{c^{-1} d}\right)^{-1}=\{c, a\} \in K_{2}(R) .
$$


Proof. The first two statements are clear. For the third, recall that

$$
h_{21}(a)=x_{21}^{a} x_{12}^{-a^{-1}} x_{21}^{a} x_{21}^{-1} x_{12}^{1} x_{21}^{-1} \text { and } w_{21}(c)=x_{21}^{c} x_{12}^{-c^{-1}} x_{21}^{c}
$$

are liftings of $\left(\begin{array}{cc}a^{-1} & 0 \\ 0 & a\end{array}\right)$ and $\left(\begin{array}{cc}0 & -c^{-1} \\ c & 0\end{array}\right)$, respectively, to St $(R)$, Using [15, Corollary 9.4 and Lemma 9.6], we get:

$$
\begin{aligned}
& \left(x_{21}^{c a^{-1}} h_{21}(a)^{-1} x_{12}^{a^{-1}} b\right)\left(x_{12}^{a c^{-1}} w_{21}(c) x_{12}^{c^{-1} d}\right)^{-1} \\
& =x_{21}^{c a^{-1}} h_{21}(a)^{-1} x_{12}^{-a^{-1} c^{-1}} w_{21}(c)^{-1} x^{-a c^{-1}} \quad\left(a^{-1} b-c^{-1} d=-a^{-1} c^{-1}\right) \\
& =h_{21}(a)^{-1} x_{21}^{a c} x_{12}^{-a^{-1} c^{-1}} x_{21}^{a c} w_{21}(c)^{-1} \\
& =h_{21}(a)^{-1} w_{21}(a c) w_{21}(c)^{-1}=w_{21}(a c) w_{21}(c)^{-1} h_{21}(a)^{-1} \\
& =h_{21}(a c) h_{21}(c)^{-1} h_{21}(a)^{-1}=\{c, a\} .
\end{aligned}
$$

This is now applied to lift the image of the transfer map via $\partial$. The next proposition, while it does not cover all cases, will suffice for most purposes here.

Proposition 2.6. Let $1 \rightarrow C_{p^{n}} \rightarrow \widetilde{G} \stackrel{\beta}{\rightarrow} G \rightarrow 1$ be a central extension of finite groups, and let $z \in \operatorname{Ker}(\beta)$ be a generator. Write $\mathrm{Q} \widetilde{G}=Q G \times R$, where $R$ is the product of all simple components of $\mathrm{Q} \widetilde{G}$ where $z \neq 1$. Consider the induced homomorphisms

$$
K_{1}(\mathrm{Z} G) \stackrel{i_{*}}{\longleftrightarrow} K_{1}\left(\hat{Z}_{p} G\right) \stackrel{\beta_{*}}{K_{1}}\left(\hat{Z}_{p} \tilde{G}\right) .
$$

Then, for any $u \in K_{1}(\mathrm{ZG})$ and any $\tilde{u} \in \beta_{*}^{-1}\left(i_{*}(u)\right)$,

$$
\beta_{z}^{\#}(u)=\partial(1,\{1-z, \tilde{u}\}) \in \mathrm{Cl}_{1}(Z \tilde{G}) .
$$

Here, $\partial: C_{p}(\mathbb{Q} \tilde{G}) \rightarrow \mathrm{Cl}_{1}(\mathrm{Z} \tilde{G})$ is the boundary map of Theorem 2.1, and

$$
(1,\{1-z, \tilde{u}\}) \in C_{p}(\mathrm{Q} G) \times C_{p}(R)=C_{p}(\mathrm{Q} \tilde{G}) .
$$

Proof. Write $u=[M]$ for some $M \in G_{n}(Z G)$. Fix liftings $A, B \in M_{n}(Z \widetilde{G})$ of $M$ and $M^{-1}$, respectively, and chooce $X \in M_{n}(Z \tilde{G})$ such that $A B-(1$ $-z) X=I$. Then, by definition,

$$
\beta_{z}^{\#}(u)=\left[\begin{array}{cc}
A & X \\
1-z & B
\end{array}\right] .
$$

Recall that $\operatorname{Ker}(\beta)$ is a $p$-group. Hence:

(1) $Z[\tilde{G}][1 / p]=Z[1 / p][G] \times \mathfrak{U}$ for some $Z[1 / p]$-order $\mathfrak{Y} \subseteq R$.

(2) $A$ is invertible in $M_{n}\left(\hat{Z}_{p} \tilde{G}\right)$ (since $\beta(A)$ is invertible).

Furthermore, $A$ is invertible in $Z[1 / p][G]$; and $1-z$ is invertible in $\mathfrak{A}$ since 
$1 / p \in \mathfrak{U}$ and $z$ has order $p^{i}>1$ in each component of $R$ (see, e.g., [31, Proposition 7-4-1]. Using Lemma 2.5, one gets corresponding liftings of $\left(\begin{array}{cc}A & X \\ 1-z & B\end{array}\right)$ to

$$
X_{1} \in \operatorname{St}_{2}\left(M_{n}\left(\hat{Z}_{p} \widetilde{G}\right)\right) \text { and } X_{2} \in \operatorname{St}_{2}\left(M_{n}(\mathrm{Z} \widetilde{G}[1 / p])\right) \text {. }
$$

Note that $\operatorname{Im}\left(\beta_{z}^{\#}\right)$ is a $p$-group by Proposition 1.4. Hence, by Theorem 2.1 (iii) and Lemma 2.5 (iii), $\beta_{z}^{\#}(u)=\partial(X)$, where

$$
X=X_{1} X_{2}^{-1}=(1,\{1-z,[A]\}) \in C_{p}(\mathrm{Q} G) \times C_{p}(R) \cong C_{p}(\mathrm{Q} \tilde{G})
$$

and $[A] \in K_{1}\left(\hat{Z}_{p} \tilde{G}\right)$ is the class of $A$. By construction,

$$
\beta_{*}([A])=i_{*}(u) \text {. }
$$

It remains to show that $[A]$ can be replaced by any $\tilde{u} \in K_{1}\left(\hat{Z}_{p} \tilde{G}\right)$ such that $\beta_{*}(\tilde{u})=i_{*}(u)$, For any such $\tilde{u}$,

$$
\tilde{u}[A]^{-1}=[1+(1-z) z] \in K_{1}\left(\hat{Z}_{p} \widetilde{G}\right)
$$

for some $x \in \hat{Z}_{p} \tilde{G}(z$ generates $\operatorname{Ker}(\beta))$. In $C_{p}(\mathrm{Q} \widetilde{G})=C_{p}(\mathrm{Q} G) \times C_{p}(R)$ :

$$
(1,\{1-z, \tilde{u}\})=(1,\{1-z, 1+(1-z) x\}) \cdot(1,\{1-z,[A]\}) .
$$

By Lemma 2.5 again, if $x \in Z \widetilde{G}$, then

$$
\partial(1,\{1-z, 1+(1-z) x\})=\left[\begin{array}{cc}
1+(1-z) x & x \\
1-z & 1
\end{array}\right]=1 ;
$$

and by continuity this holds for all $x \in \hat{Z}_{p} \tilde{G}$. So

$$
\partial(1,\{1-z, \tilde{u}\})=\partial(1,\{1-z,[A]\})=\beta_{z}^{\#}(u) .
$$

In order to apply the Artin-Hasse formulas to the symbols arising through Proposition 2.6, it is necessary to be able to calculate logarithms of the units which arise there. In practice, this will be done by describing units via their logarithms.

Fix a prime $p$, and let $A$ be the ring of integers in some finite unramified extension $F$ of $\hat{\mathrm{Q}}_{p}$. Let $\pi$ be a $p$-group, and let $I(A \pi) \subseteq A \pi$ be the augmentation ideal. By [18, Proposition 3], the logarithm series converges on $1+(p A+I(A \pi))$, and induces a homomorphism

$$
\log : K_{1}(A \pi) \rightarrow H_{0}(\pi ; F \pi)
$$

where $\pi$ acts on $F \pi$ by conjugation. Furthermore, if $\varphi \in \operatorname{Gal}\left(F / \hat{Q}_{p}\right)$ is the Frobenius automorphism $\left(\varphi(\lambda) \equiv \lambda^{p}(\bmod p)\right.$ for $\left.\lambda \in A\right)$; and if $\Phi \in \operatorname{End}(F \pi)$ is defined by setting

$$
\Phi\left(\sum \lambda_{i} g_{i}\right)=\sum \varphi\left(\lambda_{i}\right) g_{i}^{p}
$$

then the map 


$$
\Gamma_{A \pi}=(1-1 / p \Phi) \circ \log : K_{1}(A \pi) \rightarrow H_{0}(\pi ; F \pi)
$$

has image in $H_{0}(\pi ; A \pi)$ by [18, Proposition 10]. The following theorem lists the properties of $\Gamma_{A \pi}$ which will be needed later.

TheOREM 2.7. Let $\pi$ be any group, and let $A$ be the ring of integers in any finite unramified extension $F$ of $\hat{\mathrm{Q}}_{p}$. Let

$$
\varepsilon: A \pi \rightarrow A, \operatorname{Tr}: A \rightarrow \hat{Z}_{p}, \overline{\operatorname{Tr}}: A \rightarrow \mathrm{Z} / p, \omega: H_{0}(\pi ; A \pi) \rightarrow \pi^{\mathrm{ab}}
$$

denote the augmentation map, the trace, the trace $\bmod p$, and the map

$$
\omega\left(\sum \lambda_{i} g_{i}\right)=\prod g_{i}^{\operatorname{Tr}\left(\lambda_{i}\right)},
$$

respectively. Set $I(A \pi)=\operatorname{Ker}(\varepsilon)$. Then

(i) $\operatorname{Im}\left(\Gamma_{A \pi}\right)=\operatorname{Ker}\left[\omega: H_{0}(\pi ; A \pi) \rightarrow \pi^{\mathrm{ab}}\right]$ if $p$ is odd

$$
=\operatorname{Ker}\left[(\omega, \overline{\operatorname{Tr}} \circ \varepsilon): H_{0}(\pi ; A \pi) \rightarrow \pi^{\mathrm{ab}} \times \mathrm{Z} / 2\right] \text { if } p=2
$$

(ii) Assume $A=\hat{Z}_{p}$. For any $u \in K_{1}\left(\hat{Z}_{p} \pi\right)$, if

then

$$
\Gamma(u)=\sum_{i=1}^{k} \lambda_{i} g_{i}
$$

$$
\log (u)=\frac{p}{p-1} \cdot \sum_{i=1}^{k} \lambda_{i}+\sum_{i=1}^{k} \lambda_{i}\left[\left(g_{i}-1\right)+\frac{1}{p}\left(g_{i}^{p}-1\right)+\frac{1}{p^{2}}\left(g_{i}^{p^{2}}-1\right)+\ldots\right] .
$$

Proof. By [18, Theorem 2], we have

$$
\begin{aligned}
\operatorname{Im}\left(\Gamma_{A \pi}\right) & =\Gamma(1+p A)+\Gamma(1+I(A \pi)) \\
& =\Gamma(1+p A)+(\operatorname{Ker}(\omega) \cap I(A \pi)) .
\end{aligned}
$$

If $p$ is odd, then the exponential map converges on $p A$; so

$$
\log (1+p A)=p A \text {. }
$$

If $p=2$, then $\left.\log (1+2 \lambda) \equiv 2\left(\lambda-\lambda^{2}\right)(\bmod 4) ; \exp (4) \lambda\right)$ converges for $\lambda \in A$; and so (using also [18, Lemma 4]),

$$
\log (1+2 A)=\left\langle 2\left(\lambda-\lambda^{2}\right), 4 \lambda\right\rangle=2 \operatorname{Ker}[\overline{\operatorname{Tr}}: A \rightarrow Z / 2] .
$$

Together with the inversion formula:

$$
(1-1 / p \varphi)^{-1}(\lambda)=-p \varphi^{-1}(\lambda)-p^{2} \varphi^{-2}(\lambda)-p^{3} \varphi^{-3}(\lambda)-\ldots(\lambda \in A),
$$

(2) and (3) imply that

$$
\begin{aligned}
\Gamma(1+p A) & =A & & \text { if } p \text { is odd } \\
& =\operatorname{Ker}(\overline{T r}) & & \text { if } p=2 .
\end{aligned}
$$


Together with (1), this implies (i).

Finally, (ii) is immediate from the definition of $\Gamma$ (note that the sums in brackets are finite, since each $g_{i}$ has $p$-power order).

The next two propositions will make it possible to apply the Artin-Hasse formulas to certain symbols involving $u \in K_{1}\left(\hat{Z}_{p} \pi\right)$, based on knowing $\Gamma(u)$ rather than $u$ or $\log (u)$.

Proposition 2.8. Fix a prime $p$ and a finite group $G$, let $B$ be a simple component of $\mathrm{Q} G$ with center $K$, and let $F \subseteq K$ be any subfield. Let $J\left(\hat{Z}_{p} G\right)$ denote the Jacobson radical. Let $\mathrm{pr}: \hat{\mathrm{Q}}_{p} G \rightarrow \hat{B}_{p}$ denote the projection, and let $\mathrm{nr}_{B / \mathbf{K}}, N_{K / \mathbf{F}}, \operatorname{tr}_{B / \mathbf{K}}$, and $\operatorname{Tr}_{\mathbf{K} / \mathbf{F}}$ denote the (reduced) norm and (reduced) trace maps. Then, for any $u \in 1+J\left(\hat{Z}_{p} G\right)$, with

$$
\begin{aligned}
\log (u) & =\sum_{i=1}^{k} \lambda_{i} g_{i} \in \hat{Q}_{p} G \\
\log \left(N_{K / F} \operatorname{nr}_{B / K}(\operatorname{pr}(u))\right) & =\operatorname{Tr}_{K / F} \operatorname{tr}_{B / K}(\operatorname{pr}(\log (u))) \\
& =\frac{1}{[B: K]^{1 / 2}} \cdot \sum_{i=1}^{k} \lambda_{i} \chi_{B, F}\left(g_{i}\right) \in \hat{F}_{p} .
\end{aligned}
$$

Here, $\chi_{B, F}$ denotes the character of $B$ as an FG-module. In particular the expressions in (1) depend only on the value of $\log (u)$ in $H_{0}\left(G: \hat{Q}_{p} G\right)$.

Proof. Since $\hat{F}_{p}$ is torsion free, it is enough to prove this for $u^{N}$ for any $N \neq 0$. In particular, since $(\mathrm{Z} / p[G])^{*}$ is finite, it suffices to prove the result when $u \in 1+p \hat{Z}_{p} G$. By definition of the norm and trace maps, it suffices to show that

$$
\log (\operatorname{det}(1+p M))=\operatorname{trace}(\log (1+p M)) \in L,
$$

when $M \in M_{n}(A)$, and $A$ is the ring of integers in any finite extension $L$ of $\mathrm{Q}_{p}$. But this is standard: just note that

$$
\begin{aligned}
\log (\operatorname{det}(1+p M)) & =\lim _{n \rightarrow \infty}\left[p^{-n} \operatorname{trace}\left((1+p M)^{p^{n}}-1\right)\right] \\
& =\operatorname{trace}(\log (1+p M)) .
\end{aligned}
$$

The last formula (involving $\chi_{B, F}$ ) now follows directly from the definition of the character; and the fact that $[B: K]^{1 / 2} \cdot \operatorname{tr}_{B / K}$ is the ordinary trace for $B$ over $K$ [24, formula (9.7)].

Proposition 2.9. Fix a prime $p$ and a p-group $\pi$.

(i) Let $\chi \neq 1$ be a linear character of $\pi$, and let $\zeta=\zeta_{p_{n}}(n \geqq 1)$ be a generator of $\operatorname{Im}(\chi)$. Fix $u \in K_{1}\left(\hat{Z}_{p} \pi\right)$, and write 


$$
\Gamma_{\pi}(u)=\sum_{i=1}^{k} \lambda_{i} g_{i} ; \chi\left(g_{i}\right)=\zeta^{s_{i}}
$$

Then $\left(1-\zeta, \chi_{*}(u)\right)_{\mathrm{Q}(\chi)}=\zeta^{R}$, where

$$
R=\frac{1}{2} \sum_{i=1}^{k} \lambda_{i}+\sum_{i=1}^{k} \lambda_{i}\left[s_{i} / p^{n}\right]-1 / p^{n} \sum_{i=1}^{k} \lambda_{i} s_{i}
$$

(and $[x]$ denotes the greatest integer in $x$ ).

(ii) Let $B$ be a simple summand of $\mathrm{Q} \pi$ such that $B \cong M_{m}(\mathrm{Q}(\zeta))$ for some $\zeta$ $=\zeta_{p_{n}}$ with $p^{n}>2$. Let $V$ be the irreducible B-module, and define

$$
F: H_{0}\left(\pi ; \hat{Z}_{p} \pi\right) \rightarrow \hat{Z}_{p} \text { by } F\left(\sum \lambda_{i} g_{i}\right)=\sum \lambda_{i} \operatorname{dim}_{Q \zeta}\left(V^{g_{i}}\right)
$$

(where $V^{g_{i}} \subseteq V$ is the subspace fixed by $g_{i}$ ). Let $\mathrm{pr}: \hat{\mathrm{Q}}_{p} \pi \rightarrow \hat{B}_{p}$ denote the projection. Then, for any $u \in K_{1}\left(\hat{Z}_{p} \pi\right)$,

$$
\begin{aligned}
\left(\zeta, \mathrm{nr}_{B / \mathrm{Q} \zeta}(\operatorname{pr}(u))\right)_{\mathrm{Q} \zeta} & =\zeta(\Gamma(u)) & & \text { if } p \text { is odd } \\
& =(-\zeta)^{F(\Gamma(u))} & & \text { if } p=2 .
\end{aligned}
$$

Proof. (i) We may assume $\pi$ is abelian. By Theorem 2.7,

$$
\log (u)=\frac{p}{p-1} \sum_{i=1}^{k} \lambda_{i}+\sum_{i=1}^{k} \lambda_{i}\left[\left(g_{i}-1\right)+1 / p\left(g_{i}^{p}-1\right)+1 / p^{2}\left(g_{i}^{p^{2}}-1\right)+\ldots\right]
$$

and so

$$
\log \left(\chi_{*}(u)\right)=\frac{p}{p-1} \cdot \sum_{i=1}^{k} \lambda_{i}+\sum_{i=1}^{k}\left[\left(\zeta^{s_{i}}-1\right)+1 / p\left(\zeta^{p s_{i}}-1\right)+\ldots\right] .
$$

So by Theorem 2.3, $\left(1-\zeta, \chi_{*}(u)\right)_{\mathrm{Q} \zeta}=\zeta^{R}$, where

$$
\begin{gathered}
R=\frac{-1}{p^{n}} \operatorname{Tr}\left[\frac { \zeta } { 1 - \zeta } \cdot \left(\frac{p}{p-1} \sum_{i=1}^{k} \lambda_{i}+\sum_{i=1}^{k} \lambda_{i}\left[\left(\zeta^{p s_{i}}-1\right)+1 / p\left(\zeta^{p s_{i}}-1\right)\right.\right.\right. \\
+\ldots])] .
\end{gathered}
$$

Here, $\operatorname{Tr}: \hat{\mathrm{Q}}_{p} \zeta \rightarrow \hat{\mathrm{Q}}_{p}$ denotes the trace map,

$$
\begin{aligned}
1 / p^{n} \operatorname{Tr}\left(\zeta^{i}\right) & =1-1 / p & & \text { if } p^{n} \mid i \\
& =-1 / p & & \text { if } p^{n-1} \mid i \text { but } p^{n} \times i \\
& =0 & & \text { if } p^{n-1} \Varangle i .
\end{aligned}
$$

Note that

$$
-p^{n} \zeta /(1-\zeta)=\zeta^{2}+2 \zeta^{3}+3 \zeta^{4}+\ldots+\left(p^{n}-1\right) \zeta^{p^{n}} .
$$


It follows that

$$
\begin{aligned}
& \frac{-1}{p^{n}} \operatorname{Tr}(\zeta /(1-\zeta))=p^{-2 n} \operatorname{Tr}\left(\zeta^{2}+2 \zeta^{3}+\ldots\right) \\
& =\frac{1}{p^{n}}\left[(-1 / p)\left(\left(p^{n-1}-1\right)+\ldots+\left((p-1) p^{n-1}-1\right)\right)+(1-1 / p)\left(p^{n}-1\right)\right] \\
& =\frac{p-1}{2 p}
\end{aligned}
$$

Also, for any $0<s \in Z$,

$$
\frac{-1}{p^{n}} \operatorname{Tr}\left(\frac{\zeta}{1-\zeta}\left(\zeta^{s}-1\right)\right)=\frac{1}{p^{n}} \operatorname{Tr}\left(\zeta+\zeta^{2}+\ldots+\zeta^{s}\right)=\left[s / p^{n}\right]-1 / p\left[s / p^{n-1}\right]
$$

Since the last expression depends only on $s\left(\bmod p^{n}\right)$, this holds for all $s \in Z$. It follows that

$$
\begin{aligned}
& -\frac{1}{p^{n}} \operatorname{Tr}\left(\frac{\zeta}{1-\zeta}\left[\left(\zeta^{s}-1\right)+1 / p\left(\zeta^{p s}-1\right)+\ldots\right]\right) \\
& \quad=\left(\left[\frac{s}{p^{n}}\right]-\frac{1}{p}\left[\frac{s}{p^{n-1}}\right]\right)+\left(\frac{1}{p}\left[\frac{s}{p^{n-1}}\right]-\frac{1}{p^{2}}\left[\frac{s}{p^{n-2}}\right]\right)+\ldots=\left[\frac{s}{p^{n}}\right]-\frac{s}{p^{n}} .
\end{aligned}
$$

The formula for $R$ now follows upon substituting (3) and (4) into (1).

(ii) Fix $u \in K_{1}\left(\hat{Z}_{p} \pi\right)$, and write $\Gamma_{\pi}(u)=\sum_{i=1}^{k} \lambda_{i} g_{i}$. Then

$$
\log (u)=\frac{p}{p-1} \sum \lambda_{i}\left[\left(g_{i}-1\right)+\frac{1}{p}\left(g_{i}^{p}-1\right)+\ldots\right]
$$

by Theorem 2.7. So by Proposition 2.8,

$$
\begin{aligned}
& \log \left(\operatorname{nr}_{B / \mathrm{Q} \zeta}(\operatorname{pr}(u))\right)=\operatorname{tr}_{B / \mathrm{Q} \zeta}(\operatorname{pr}(\log (u))) \\
& \quad=\frac{p m}{p-1} \sum \lambda_{i}+\sum \lambda_{i}\left[\left(\chi\left(g_{i}\right)-m\right)+\frac{1}{p}\left(\chi\left(g_{i}^{p}\right)-m\right)+\ldots\right],
\end{aligned}
$$

where $\chi: \pi \rightarrow Q \zeta$ is the character of $V$ (recall that $\chi(1)=m$ ).

Fix $g \in \pi$, and let $\left\{\zeta_{1}, \zeta_{2}, \ldots, \zeta_{m}\right\}$ be the eigenvalues for $g$. If $\left|\zeta_{i}\right|=p^{u}$, then (using (2) above)

$$
\begin{array}{rlr}
\frac{1}{p^{n}} \operatorname{Tr}\left[\frac{p}{p-1}+\left(\zeta_{i}-1\right)+\frac{1}{p}\left(\zeta_{i}^{p}-1\right)+\frac{1}{p^{2}}\left(\zeta_{i}^{p^{2}}-1\right)+\ldots\right] & \\
\quad=\left[\frac{p}{p-1}-1-\frac{1}{p}-\ldots-\frac{1}{p^{u-1}}\right]\left[1-\frac{1}{p}\right]+\left[\frac{1}{p^{u-1}}\right]\left[-\frac{1}{p}\right]=0 \text { if } u \geqq 1 \\
\quad=\left(\frac{p}{p-1}\right)\left(1-\frac{1}{p}\right)=1 & \text { if } u=0 .
\end{array}
$$


It follows that

$$
\frac{1}{p^{n}} \operatorname{Tr}\left[\frac{p m}{p-1}+(\chi(g)-m)+1 / p\left(\chi\left(g^{p}\right)-m\right)+\ldots\right]=\operatorname{dim}_{\mathrm{Q}_{\zeta}}\left(V^{g}\right)
$$

for $g \in \pi$; and hence that

$$
p^{-n} \operatorname{Tr}\left(\log \left(\mathrm{nr}_{B / \mathrm{Q} \zeta}(\operatorname{pr}(u))\right)\right)=\sum \lambda_{i} \operatorname{dim}_{\mathrm{Q} \zeta}\left(V^{g_{i}}\right) .
$$

The result now follows from the Artin-Hasse formula (Theorem 2.3).

Since the algebraic structure of the rational group rings will sometimes play a role in calculations, we note the following facts.

THEOREM 2.10. (i) If $\pi$ is a finite group of exponent $n$, then the center of any simple component of $\mathrm{Q} \pi$ is isomorphic to a subfield of $\mathrm{Q} \zeta_{n}$. In particular, for any prime $p|| \pi \mid, C_{p}(\mathrm{Q} \pi)$ has exponent dividing $n$.

(ii) If $\pi$ is a p-group, where $p$ is an odd prime, then $\mathrm{Q} \pi$ is a product of matrix rings over fields $Q \zeta_{p^{k}}$ for various $k$.

(iii) If $\pi$ is a 2-group, then $\mathrm{Q} \pi$ is a product of matrix rings over division algebras isomorphic to

$$
\mathrm{Q} \zeta_{2^{k}}, \mathrm{Q}\left[\zeta_{2^{k}}+\zeta_{2^{k}}^{-1}\right], \mathrm{Q}\left[\zeta_{2^{k}}-\zeta_{2^{k}}^{-1}\right] \text {, or } \mathrm{Q} \zeta_{2^{k}}[j] \text {. }
$$

Proof. The first statement follows from [10, Theorem 15.16] (and Theorem 2.2). For the second statement, see [25, Sections 2 and 3].

Finally, the following result will be needed in Section 4.

Proposition 2.11. Fix a prime $p$, and fix $n \geqq 1$ such that $p \nmid n$. Then, for any abelian p-group of rank $\leqq 2$ the transfer map

$$
t: \mathrm{Cl}_{1}\left(\mathrm{Z} \zeta_{n}[\pi]\right) \rightarrow \mathrm{Cl}_{1}(\mathrm{Z} \pi)
$$

is an isomorphism.

Proof. This follows directly from results in [1, Section 2]. Alternatively, if $p$ is odd, the result is shown in [20, Theorem 4.3]. If $p=2$, then the transfer map

$$
\operatorname{trf}: C_{2}\left(\mathrm{Q} \zeta_{n}[\pi]\right) \rightarrow C_{2}(\mathrm{Q} \pi)
$$

is onto by [15, Corollary A.15]; and so $t$ is onto by the localization sequence of Theorem 2.1. Let $\mathfrak{M} \subseteq \mathrm{Q}$ be the maximal order, and consider the maps 


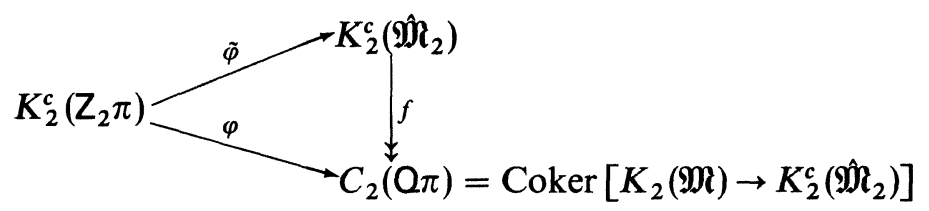

where $\tilde{\varphi}, \varphi$ are induced by the inclusion $\hat{Z}_{2} \pi \subseteq \hat{\mathfrak{M}}_{2}$, and $f$ is the projection. Then, by [20, Theorem 4.4] (and Theorem 1.1]:

$$
\mathrm{Cl}_{1}\left(\mathrm{Z} \zeta_{n}[\pi]\right)=\operatorname{Coker}(\tilde{\varphi}) \text { and } \mathrm{Cl}_{1}(\mathrm{Z} \pi) \cong \operatorname{Coker}(\varphi) \text {. }
$$

Since $t$ is onto, it will suffice to show that these cokernels are abstractly isomorphic, or that $\operatorname{Ker}(f) \subseteq \operatorname{Im}(\tilde{\varphi})$.

Write $\mathrm{Q} \pi=\prod_{i=1}^{k} F_{i}$, where for each $i, F_{i} \cong Q \zeta_{2^{n}}$ for some $n$. Then, by Theorem 2.2,

$$
\operatorname{Ker}(f)=\prod_{i}\left\{K_{2}^{c}\left(\hat{Z}_{2}\right): F_{i} \cong \mathbf{Q}\right\} \cong \prod_{i}\left\{( \pm 1): F_{i} \cong \mathbf{Q}\right\} .
$$

Note also that for any $n \geqq 2$, and $\zeta=\zeta_{2^{n}}$, then in $K_{2}^{c}\left(\hat{Z}_{2} \zeta_{2^{n}}\right)$ :

$$
\{\zeta, \zeta\}=\{\zeta,-\zeta\}^{1+2^{n-1}}=1 \text {. }
$$

Thus, since $\operatorname{rk}(\pi) \leqq 2, \operatorname{Ker}(f)$ is generated by elements $\tilde{\varphi}(\{ \pm g, \pm h\})$ for $f, h \in \pi$. Hence $\operatorname{Ker}(f) \subseteq \operatorname{Im}(\tilde{\varphi})$, and $\mathrm{Cl}_{1}\left(\mathrm{Z} \zeta_{n}[\pi]\right) \cong \mathrm{Cl}_{1}(\mathrm{Z} \pi)$.

3.

Concrete examples of central extensions $1 \rightarrow\langle z\rangle \rightarrow \tilde{G} \stackrel{\beta}{\rightarrow} G \rightarrow 1$ of $p$ groups for which $\beta_{z}^{\#} \neq 1$ - in fact, for which $\beta_{z}^{\#}\left(S K_{1}(Z G)\right) \neq 1$ - will now be constructed. The determination of $\operatorname{Ker}\left(\beta_{z}^{\#}\right)$ for arbitrary $\beta$ is at best an extremely tedious combinatorial problem, where (as indicated by Theorems 2.1 and 2.2) the chief difficulty is to describe the image of $K_{2}\left(\hat{Z}_{p} \tilde{G}\right)$ in $C_{p}(Q \tilde{G})$.

So instead, for fixed $\beta$ as above, we consider the family of pushout sequences

$$
1 \rightarrow\left\langle z_{s}\right\rangle \rightarrow \tilde{G}_{s} \stackrel{\beta_{s}}{\rightarrow} G \rightarrow 1
$$

for $s \geqq 0$, where $\tilde{G}_{s}=\left\langle\widetilde{G}, z_{s}:\left[\widetilde{G}, z_{s}\right]=1,\left(z_{s}\right)^{s^{s}}=z\right\rangle$. For any $0 \leqq s \leqq t$, regard $\tilde{G}_{s}$ as a subgroup of $\tilde{G}_{t}\left(\right.$ where $z_{s}=\left(z_{t}\right)^{t^{t-s}}$ ). The diagram

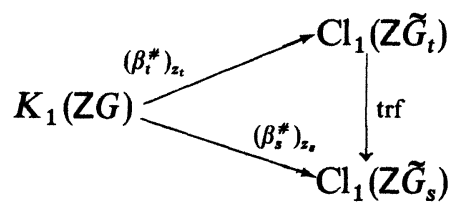


commutes (see [16, Section 6]); so the $\operatorname{Ker}\left(\left(\beta_{s}^{\#}\right)_{z_{s}}\right)$ are non-increasing. For large $s$, the symbols $\left\{z_{s},-\right\}$ come to "dominate" in $K_{2}\left(\hat{Z}_{p}\left[\tilde{G}_{s}\right]\right)$ in a way made more precise in Proposition 3.2. Sufficiency conditions for showing $\left(\beta_{s}^{\#}\right)_{z_{s}}\left(S K_{1}(Z G)\right) \neq 1$ are then given in Theorem 3.3 below, after which specific examples are worked out in Propositions 3.4 and 3.5.

LEMMA 3.1. Let $\beta: \widetilde{G} \longrightarrow G$ be a central extension of p-groups, for some prime $p$, and let $H \subseteq \tilde{G}$ be a subgroup such that $\beta(H)=G$. Then

$$
\operatorname{Im}\left[K_{2}^{c}\left(\hat{Z}_{p} H\right) \rightarrow K_{2}^{c}\left(\hat{Z}_{p} G\right)\right]=\operatorname{Im}\left[K_{2}^{c}\left(\hat{Z}_{p} \tilde{G}\right) \rightarrow K_{2}^{c}\left(\hat{Z}_{p} G\right)\right] .
$$

Proof. Let $\alpha=\beta \mid H$. By [21, Proposition 2.1], there is the following commutative diagram with exact rows:

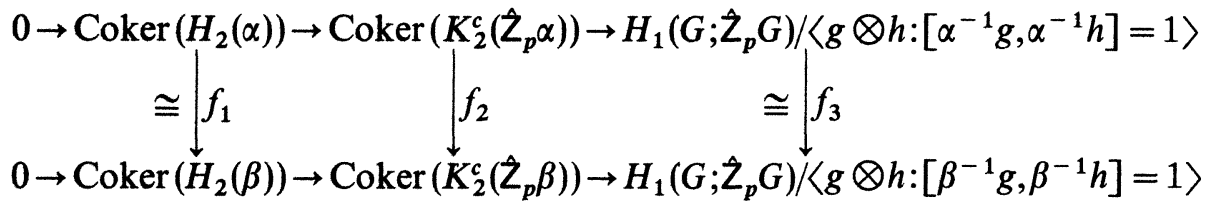

where $f_{1}, f_{2}, f_{3}$ are all induced by the inclusion $H \subseteq \tilde{G}$. That $f_{3}$ is an isomorphism is immediate. That $f_{1}$ is an isomorphism follows from the five-term exact sequences

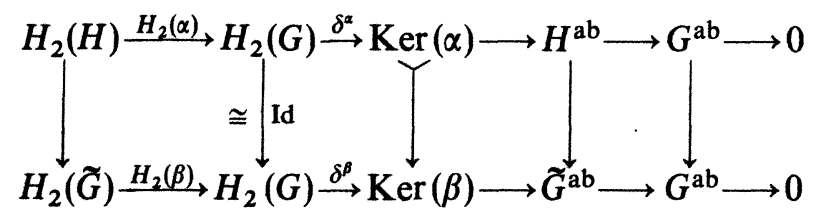

(see, e.g., [27, Section II.3]). So $f_{2}$ is injective, and $K_{2}^{c}\left(\hat{Z}_{p} \alpha\right)$ and $K_{2}^{c}\left(\hat{Z}_{p} \beta\right)$ have the same image.

This is used to show:

Proposition 3.2. Fix p, let $G$ be a p-group, and let $H \triangleleft G$ and $z \in Z(G)$ be such that $G=\langle H, z\rangle$. Set $q=\exp (H)$, and let $\tilde{\varphi}$ denote the composite

$$
\tilde{\varphi}: K_{2}^{c}\left(\hat{Z}_{p} G\right) \stackrel{\varphi_{G}}{\longrightarrow} C_{p}(\mathrm{Q} G) \longrightarrow C_{p}(\mathrm{Q} G) /_{q} C_{p}(\mathrm{Q} G)
$$

(i.e., dividing out by q-torsion). Then

$$
\tilde{\varphi}\left(K_{2}^{c}\left(\hat{Z}_{p} G\right)\right)=\langle\tilde{\varphi}(\{z, 1+(1-z) g\}): g \in G\rangle .
$$

Proof. Set $G^{\prime}=G / z$. By Lemma 3.1,

$$
\operatorname{Im}\left[K_{2}^{c}\left(\hat{Z}_{p} G\right) \rightarrow K_{2}^{c}\left(\hat{Z}_{p} G^{\prime}\right)\right]=\operatorname{Im}\left[K_{2}^{c}\left(\hat{Z}_{p} H\right) \rightarrow K_{2}^{c}\left(\hat{Z}_{p} G^{\prime}\right)\right] .
$$


By [21, Theorem 1.4],

$\operatorname{Ker}\left[K_{2}^{c}\left(\hat{Z}_{p} G\right) \rightarrow K_{2}^{c}\left(\hat{Z}_{p} G^{\prime}\right)\right]=\langle\{g, 1+(1-z) h\}: g, h \in G, g h=h g\rangle$.

It follows that

That

$$
\begin{aligned}
K_{2}^{c}\left(\hat{Z}_{p} G\right)=K_{2}^{c}\left(\hat{Z}_{p} H\right) & +\langle\{h, 1+(1-z) g\}: h \in H, h g=g h\rangle \\
& +\langle\{z, 1+(1-z) g\}: g \in G\rangle .
\end{aligned}
$$

$$
\exp (\langle\{h, 1+(1-z) g\}: h \in H, g \in H, h g=g h\rangle) \mid \exp (H)=q
$$

is clear. By Theorem 2.10 (and Theorem 2.2),

$$
\exp \left(\varphi_{G}\left(K_{2}^{c}\left(\hat{Z}_{p} H\right)\right)\right)\left|\exp \left(C_{p}(\mathrm{Q} H)\right)\right| \exp (H)=q .
$$

Hence

$$
\varphi\left(K_{2}^{c}\left(\hat{Z}_{p} G\right)\right) \subseteq\langle\varphi(\{z, 1+(1-z) g\}): g \in G\rangle+{ }_{q} C_{p}(\mathrm{Q} G) ;
$$

and the result follows.

If $1 \rightarrow C \rightarrow \widetilde{G} \stackrel{\beta}{\rightarrow} G \rightarrow 1$ is any central extension of $p$-groups, then we define an isomorphism

$$
x=x_{\beta}: \frac{C \cap[\tilde{G}, \tilde{G}]}{\langle z \in C: z=[g, h], g, h \in \widetilde{G}\rangle} \cong \operatorname{Coker}\left[S K_{1}(\mathrm{Z} \beta): S K_{1}(\mathrm{Z} \tilde{G}) \rightarrow S K_{1}(\mathrm{ZG})\right]
$$

as follows. For any $w \in C \cap[\tilde{G}, \tilde{G}]$, choose $\tilde{u} \in K_{1}\left(\hat{Z}_{p} \tilde{G}\right)$ such that $\Gamma_{\tilde{G}}(\tilde{u})=$ $1-w$ and $\beta_{*}(u) \in S K_{1}\left(\hat{Z}_{p} G\right)$; then $x(w)$ is represented by any lifting of $\beta_{*}(u)$ to $S K_{1}(Z G)$. That this defines an isomorphism to $\operatorname{Coker}\left(S K_{1}\left(Z_{p} \beta\right)\right)$ is shown in [18, Proposition 16]. That

$$
\operatorname{Coker}\left(S K_{1}\left(\hat{Z}_{p} \beta\right)\right) \cong \operatorname{Coker}\left(S K_{1}(Z \beta)\right)
$$

follows from the exact sequence of Theorem 2.1(2), and the fact that $\mathrm{Cl}_{1}(\mathrm{Z} \beta)$ is onto [17, Lemma 1$]$.

THEOREM 3.3. Fix $p$, let $\tilde{G}$ be a p-group, and fix $H \triangleleft \tilde{G}$ and $z \in Z(\widetilde{G})$ such that $\widetilde{G}=\langle H, z\rangle$. Fix $w=z^{p^{r}} \in[\widetilde{G}, \widetilde{G}]$. Set $G=\widetilde{G} / z$, let $\beta: \widetilde{G} \rightarrow G$ be the projection, and let

$$
\beta^{\#}=\beta_{z}^{\#}: K_{1}(\mathrm{ZG}) \rightarrow \mathrm{Cl}_{1}(\mathrm{Z} \tilde{G})
$$

be the induced transfer homomorphism.

Set $q=\exp (H)$, and let $S$ be a set of irreducible complex characters of $\tilde{G}$ such that for each $\chi \in S, z^{q}$ acts non-trivially on the corresponding irreducible $\mathrm{Q} \tilde{G}$-representation $V_{\chi}$. For each $\chi \in S$, set 


$$
\begin{aligned}
& p^{k_{x}}=\left(\text { order of } z^{q} \text { acting on } V_{\chi}\right)>1 \\
& p^{m_{x}}=\left(\text { order of } w \text { acting on } V_{\chi}\right)=\max \left\{1, \mathrm{p}^{k_{x}-r}\right\} \\
& p^{n_{x}}=\operatorname{dim}_{\mathrm{C}}\left(V_{\chi}\right) .
\end{aligned}
$$

Define a homomorphism

$$
F: Z \widetilde{G} \rightarrow \prod_{\chi \in S} \mathrm{Z} / p^{k_{x}}
$$

by setting, for $g \in \widetilde{G}$,

$$
F(g)=\left(\operatorname{dim}_{C}\left(V_{\chi}^{g}\right)\right)_{\chi \in S}\left(V_{\chi}^{g}=\left\{v \in V_{\chi}: g v=v\right\}\right) .
$$

Finally, define

$$
X=\left(X_{\chi}\right)_{\chi \in S} \in \prod_{\chi \in S} \mathrm{Z} / p^{k_{\chi}}
$$

by setting

$$
X_{\chi}= \begin{cases}p^{n_{\chi}-m_{\chi}} & \text { if } m_{\chi}>0 \\ 0 & \text { if } \left.m_{\chi}=0 \text { (i.e., if } w \text { fixes } V_{\chi}\right) .\end{cases}
$$

Then $\beta^{\#}\left(x_{\beta}(w)\right) \neq 1$ if $X \notin \operatorname{Im}(F)$. More generally, $\beta^{\#}\left(S K_{1}(Z G)\right)$ is cyclic of order at least equal to the order of $X$ in $\operatorname{Coker}\left(F \circ \Gamma_{\tilde{G}}\right)$.

Proof. We can clearly assume $H \neq 1$; that is, $q>1$. Since $z \in Z(\tilde{G}), z$ acts on each $V_{x}$ via multiplication by same root of unity $\zeta_{x}$, where $|\zeta|>q$ by assumption. Since $q=\exp (H)<\left|\zeta_{\chi}\right|$, this shows that all eigenvalues for elements of $H$, and hence of $G$, acting om $V_{\chi}$, lie in $\left\langle\zeta_{\chi}\right\rangle$. Thus for $\chi \in S$,

$$
\mathrm{Q} \chi=\mathrm{Q}\left(\zeta_{\chi}\right) \text { and }\left|\zeta_{\chi}\right|=q p^{k_{x}} \geqq p^{2} \text {. }
$$

Hence, we can define a homomorphism

$$
R=\prod R_{\chi}: C_{p}(\mathrm{Q} G) \rightarrow \prod_{\chi \in S} \mathrm{Z} / p^{k_{x}}
$$

as follows. Each $\chi$ corresponds to a simple summand $B_{\chi}$ of $Q \tilde{G}$ with center Q ; let

$$
\mathrm{pr}_{\chi}: \mathrm{Q} \tilde{G} \longrightarrow B_{\chi}
$$

be the projection map. Then $R_{\chi}$ is defined to be the composite

$$
R_{\chi}: C_{p}(\mathrm{Q} \tilde{G}) \stackrel{\left(\mathrm{pr}_{\chi}\right)_{a}}{\longrightarrow} C_{p}\left(B_{\chi}\right) \stackrel{\lambda_{x}}{\cong}\left\langle\zeta_{\chi}\right\rangle \stackrel{\left(\zeta_{\chi}\right)^{a} \rightarrow a}{\longrightarrow} \mathrm{Z} / p^{k_{x}} .
$$

Here, $\lambda_{x}=\lambda_{B_{x}}$ is the norm residue symbol isomorphism of Theorem 2.2.

Now consider the maps 


$$
\begin{gathered}
K_{2}\left(\hat{Z}_{p} \widetilde{G}\right) \stackrel{\varphi}{\rightarrow} C_{p}(\mathrm{Q} \tilde{G}) \stackrel{\partial}{\rightarrow} \mathrm{Cl}_{1}(\mathrm{Z} \widetilde{G}) \ni \beta^{\#}\left(\chi_{\beta}(w)\right) \\
X \in \prod_{\chi \in S}^{\downarrow} \mathrm{Z} / p^{k_{\chi}}
\end{gathered}
$$

where the top row is exact by Theorem 2.1. By definition, $\boldsymbol{x}_{\beta}(w)$ lifts to some $\tilde{u} \in K_{1}\left(\hat{Z}_{p} \widetilde{G}\right)$ with $\Gamma_{\tilde{G}}(\tilde{u})=1-w$. So by Proposition 2.6,

$$
\beta^{\#}\left(x_{\beta}(w)\right)=\partial(1,\{1-z, \tilde{u}\}) \in \mathrm{Cl}_{1}(\mathrm{Z} \tilde{G}),
$$

where, upon writing $Q \widetilde{G}=Q G \times A$,

$$
(1,\{1-z, \tilde{u}\}) \in C_{p}(\mathrm{Q} G) \oplus C_{p}(A) \cong C_{p}(\mathrm{Q} \tilde{G}) .
$$

To prove the theorem, it hus remains to show:

(2) $\operatorname{Im}(R \varphi) \subseteq \operatorname{Im}(F)$; and

(3) $R(1,\{1-z, \tilde{u}\})=X$.

STEP 1. By construction, and (1), all $q$-torsion in $C_{p}(Q \tilde{G})$ lies in $\operatorname{Ker}(R)$. So by Proposition 3.2,

$$
R \varphi\left(K_{2}\left(\hat{Z}_{p} \tilde{G}\right)\right)=\left\{R(\{z, u\}): u \in\left(\hat{Z}_{p} \tilde{G}\right)^{*}\right\} .
$$

By Proposition 2.9, for any $u \in\left(\hat{Z}_{p} G\right)^{*}$ and any $\chi \in S$,

$$
\begin{aligned}
\left(\zeta_{\chi}, \mathrm{nr}_{B_{\chi} / \mathrm{Q}_{\chi}}\left(\operatorname{pr}_{\chi}(u)\right)\right)_{\mathrm{O}_{\chi}}= & \zeta_{\chi} F_{\chi}(\Gamma(u)) \\
& \left(F_{\chi}\left(\sum \lambda_{i} g_{i}\right)=\sum \lambda_{i} \operatorname{dim}_{\mathrm{C}}\left(V_{\chi}^{\left.\left.g_{i}\right)\right) .}\right.\right.
\end{aligned}
$$

So $R(\{z, u\})=F\left(\Gamma_{\tilde{G}}(u)\right)$ for all such $u$, and by (4):

$$
R \varphi\left(K_{2}\left(\hat{Z}_{p} \tilde{G}\right)\right) \subseteq \operatorname{Im}(F) .
$$

This proves (2).

STEP 2. Recall that for any $\chi \in S, w$ acts on $V_{\chi}$ via multiplication by $\zeta_{\chi}^{p^{r}}$. Since $w \in[\tilde{G}, \tilde{G}]$, this implies that

$$
1=\operatorname{det}_{\mathrm{C}}\left(w, V_{\chi}\right)=\left(\zeta_{\chi}^{p^{r}}\right)^{p^{n_{x}}} ;\left|\zeta_{\chi}^{p^{r}}\right|=p^{m_{x}}
$$

and so $n_{\chi} \geqq m_{\chi}$. Thus

$$
X_{\chi}=p^{n_{x}-m_{x} \in Z / p^{k_{x}}}
$$

is defined when $m_{\chi}>0$.

We have $\Gamma(\tilde{u})=1-w$. By Theorem 2.7, 


$$
\begin{aligned}
\log (u) & =\left[(1-w)+1 / p\left(1-w^{p}\right)+1 / p^{2}\left(1-w^{p^{2}}\right)+\ldots\right] \\
& +\sum \lambda_{i}\left(g_{i}-g_{i}^{\prime}\right) \in \widehat{Q}_{p} \tilde{G},
\end{aligned}
$$

where $g_{i}$ is conjugate to $g_{i}^{\prime}$ for each $i$. Then, for each $\chi \in S$,

$$
\begin{aligned}
\log \left(\operatorname{det}\left(\operatorname{pr}_{\chi}(u)\right)\right) & =\operatorname{tr}\left(\operatorname{pr}_{\chi}(\log u)\right)=\operatorname{tr}\left(\operatorname{pr}_{\chi}\left((1-w)+1 / p\left(1-w^{p}\right)+\ldots\right)\right) \\
& =p^{n}\left(1-\zeta^{p}\right)+p^{n-1}\left(1-\zeta^{p^{r+1}}\right)+\ldots+p^{n-m+1}\left(1-\zeta p^{r+m-1}\right)
\end{aligned}
$$

(where we set $n=n_{\chi}, \zeta=\zeta_{\chi}$, and $m=m_{\chi}$ for short).

By definition of $R_{\chi}$,

$$
\zeta^{R_{\chi}(\{1-z, \tilde{u}\})}=\left(1-\zeta, \operatorname{det}\left(\operatorname{pr}_{\chi}(u)\right)\right)_{\mathrm{O}_{\chi}} .
$$

By the Artin-Hasse formula (Theorem 2.3), $R_{\chi}(\{1-z, \tilde{u}\})=0=X_{\chi}$ when $m=0$. If $m>0$, then $|\zeta|=p^{r+m}$, and using (5) we get:

$$
\begin{aligned}
R_{\chi}(\{1-z, \tilde{u}\}) & =-p^{-r-m} \cdot \operatorname{Tr}\left(\frac{\zeta}{1-\zeta} \cdot \log \left(\operatorname{det}\left(\operatorname{pr}_{\chi}(\tilde{u})\right)\right)\right)\left(\operatorname{Tr}=\operatorname{Tr}_{\mathrm{Q}_{\chi} / \mathrm{Q}}\right) \\
& =-p^{-r-m} \cdot \operatorname{Tr}\left(\frac{\zeta}{1-\zeta}\left[p^{n}\left(1-\zeta^{p^{r}}\right)+\ldots+p^{n-m+1}\left(1-\zeta p^{r+m-1}\right)\right]\right) \\
& =-p^{-r-m} \operatorname{Tr}\left(\sum_{j=0}^{m-1} p^{n-j}\left(\zeta+\zeta^{2}+\zeta^{3}+\ldots+\zeta^{p^{r+j}}\right)\right) \\
& =-p^{-r-m} \cdot p^{n-m+1} \cdot \operatorname{Tr}\left(\zeta^{r+m-1}\right) \quad\left(|\zeta|=p^{r+m}\right) \\
& =-p^{-r-m} \cdot p^{n-m+1} \cdot\left(-p^{r+m-1}\right)=p^{n-m}=X_{\chi} .
\end{aligned}
$$

This proves formula (3).

We now construct concrete examples to show that for any prime $p$, there are surjections of $p$-groups whose Whitehead transfer map is non-zero. This is easiest for odd $p$.

Theorem 3.4. Fix an odd prime p. Define

$$
H=\left\langle a_{1}, a_{2}, a_{3}, a_{4}:\left[a_{i},\left[a_{j}, a_{k}\right]\right]=1=a_{i}^{p}, \text { all } i, j, k\right\rangle ;
$$

and set

$$
\tilde{G}=\left\langle H, z:[z, H]=1, z^{p}=\left[a_{1}, a_{2}\right]\left[a_{3}, a_{4}\right]\right\rangle .
$$

Set $G=\tilde{G} / z$. Then the transfer map

$$
\beta^{\#}=\beta_{z}^{\#}: K_{1}(\mathrm{Z} G) \rightarrow K_{1}(\mathrm{Z} \tilde{G})
$$

for the extension $1 \rightarrow\langle z\rangle \rightarrow \tilde{G} \stackrel{\beta}{\rightarrow} G \rightarrow 1$ is non-zero. 
Proof. Note first that $H$ is a central extension of the form

$$
1 \rightarrow[H, H] \rightarrow H \stackrel{\alpha}{\rightarrow} H^{\text {ab }} \rightarrow 1,
$$

where

$$
H^{\mathrm{ab}}=\left\langle\bar{a}_{i}=\alpha\left(a_{i}\right)\right\rangle \cong\left(C_{p}\right)^{4} ;[H, H]=\left\langle\left[a_{i}, a_{j}\right]\right\rangle \cong\left(C_{p}\right)^{6} .
$$

In particular, $\langle z\rangle \cap[\tilde{G}, \tilde{G}]=\left\langle z^{p}\right\rangle$, and $p=\exp (H)$. Set $w=z^{p}$, so that in the notation of Theorem 3.3, $q=p^{r}=p$.

STEP 1. We consider subplanes - 2-dimensional subspaces - of $H^{\mathrm{ab}} \cong\left(C_{p}\right)^{4}$. Set

$$
\mathscr{P}=\left\{P \subseteq H^{\mathrm{ab}}: P \text { a subplane, } z^{p} \notin\left[H, \alpha^{-1} P\right]\right\} .
$$

Fix some $P \in \mathscr{P}$, let $\left\{\bar{x}_{1}, \bar{x}_{2}, \bar{x}_{3}, \bar{x}_{4}\right\}$ be any basis of $H^{\text {ab }}$ such that $P$ $=\left\langle\bar{x}_{1}, \bar{x}_{2}\right\rangle$, and choose elements $x_{i} \in \alpha^{-1}\left(\bar{x}_{i}\right) \subseteq H$. Note that

$$
[H, H]=\left\langle\left[x_{3}, x_{4}\right],\left[H, \alpha^{-1} P\right]\right\rangle=\left\langle z^{p},\left[H, \alpha^{-1} P\right]\right\rangle .
$$

Hence, upon replacing $x_{4}$ by $x_{4}^{i}$ for some $1 \leqq i \leqq p-1$, we may assume that

$$
\left[x_{3}, x_{4}\right] \equiv z^{p}\left(\bmod \left[H, \alpha^{-1} P\right]\right) .
$$

Define a homomorphism $\tau: \widetilde{G} \rightarrow \operatorname{GL}(p, \mathrm{C})$ by setting (here $\zeta=\zeta_{p^{2}}$, for short):

$$
\begin{gathered}
\tau(z)=\zeta \cdot I, \tau\left(x_{1}\right)=\tau\left(x_{2}\right)=\tau\left(\left[H, \alpha^{-1} P\right]\right)=I, \\
\tau\left(x_{3}\right)=\left[\begin{array}{ccccc}
0 & 1 & 0 & \ldots & 0 \\
0 & 0 & 1 & & 0 \\
\vdots & & & & \vdots \\
0 & 0 & 0 & \ldots & 1 \\
1 & 0 & 0 & \ldots & 0
\end{array}\right] \quad \tau\left(x_{4}\right)=\left[\begin{array}{ccccc}
1 & 0 & \ldots & \ldots & 0 \\
0 & \zeta^{p} & & & \vdots \\
\vdots & & \zeta^{2 p} & & \vdots \\
\vdots & & & . & \\
0 & \ldots & \ldots & \ldots & \zeta^{(p-1) p}
\end{array}\right]
\end{gathered}
$$

Let $\chi(P)$ and $V_{\chi(P)}$ be the corresponding irreducible character and representation.

For any $r, s$ not both zero $\bmod p, \tau\left(x_{3}^{r} x_{4}^{s}\right)$ has trace zero, and all eigenvalues are $p$ th roots of unity; hence the eigenvalues must be $\left\{1, \zeta^{p}, \zeta^{2 p}, \ldots, \zeta^{(p-1) p}\right\}$. The same then holds for eigenvalues of $\tau\left(g x_{3}^{r} x_{4}^{s}\right)$ for any $g \in\left\langle[H, H], x_{1}, x_{2}\right\rangle$; while if $g \in \tilde{G}-H$, then all eigenvalues of $\tau(g)$ have order $p^{2}$. It follows that for $g \in \widetilde{G}$,

$$
\begin{aligned}
\operatorname{dim}_{\mathrm{C}}\left(V_{\not P}^{g}\right) & =1 & & \text { if } g \in H, \alpha(g) \in H^{\mathrm{ab}}-P \\
& =0 \text { or } p & & \text { otherwise. }
\end{aligned}
$$


Note that while $\chi(P)$ depends on the $x_{i}$ as well as $P$, the $\operatorname{dim}\left(V_{\chi(P)}^{S}\right)$ do not.

Let

$$
S=\{\chi(P): P \in \mathscr{P}\} .
$$

Then, in the notation of Theorem 3.3, $k_{\chi}=m_{\chi}=n_{\chi}=1$ for all $\chi \in S$. Consider the map and element

$$
F: \hat{Z}_{p} \tilde{G} \rightarrow \prod_{P \in S}(\mathrm{Z} / p), \quad X \in \prod_{P \in S}(\mathrm{Z} / p)
$$

defined by

$$
F(g)=\left(\operatorname{dim}_{\mathrm{C}}\left(V_{\chi}^{g}\right)\right)_{\chi \in S} ; \quad X=\left(X_{\chi}\right)_{\chi \in S}=(1)_{\chi \in S} .
$$

By Theorem 3.3, we will be done upon showing that $X \notin \operatorname{Im}(F)$. Assume that there exists a subset $\mathscr{P}_{0} \subseteq \mathscr{P}$ such that

$$
\text { for all } 1 \neq g \in H^{\mathrm{ab}}, g \in P \text { for some unique } P \in \mathscr{P}_{0} \text {. }
$$

Then $\left|\mathscr{P}_{0}\right|=\left(p^{4}-1\right) /\left(p^{2}-1\right)=p^{2}+1$. For any $g \in \tilde{G}$,

$$
\begin{aligned}
\sum_{P \in \mathscr{P}_{0}} \operatorname{dim}\left(V_{\chi(P)}^{g}\right) & =p^{2} \cdot 1+1 \cdot 0=0 \in Z / p & & \text { if } g \in H, \alpha(g) \neq 0 \\
& =\left(p^{2}+1\right) \cdot 0=0 \in Z / p & & \text { otherwise. }
\end{aligned}
$$

On the other hand,

$$
\sum_{P \in \mathscr{P}_{0}} X_{\chi(P)}=1 \in \mathrm{Z} / p
$$

implying that $X \notin \operatorname{Im}(F)$.

STEP 3. It remains to construct $\mathscr{P}_{0} \subseteq \mathscr{P}$ satisfying (2). For convenience, we write $H^{\mathrm{ab}}$ additively here: $H^{\mathrm{ab}}=(\mathrm{Z} / p)^{4}$, where we identify $a_{1}$ $=(1,0,0,0)$, etc. Then

$$
\mathscr{P}=\left\{P \subseteq H^{\mathrm{ab}}: P \text { subplane, } a_{1} \wedge a_{2} \neq-a_{3} \wedge a_{4} \text { in } H_{2}\left(H^{\mathrm{ab}} / P\right)\right\} .
$$

Fix a quadratic nonresidue $N \in Z / p$. Define a subset $T \subseteq \mathrm{GL}(2, \mathrm{Z} / p)$ :

$$
\begin{aligned}
T & =\left\{\left(\begin{array}{cc}
a & N b \\
b & a
\end{array}\right):(a, b) \neq(0,0), a^{2}-N b^{2} \neq-1\right\} \cap \\
& \cap\left\{\left(\begin{array}{cc}
a & -N b \\
b & -a
\end{array}\right): a^{2}-N b^{2}=-1\right\} .
\end{aligned}
$$

For each $\varphi \in T$, set 


$$
P_{\varphi}=\left\{(x, \varphi(x)) \in(\mathrm{Z} / p)^{4}: x \in(\mathrm{Z} / p)^{2}\right\} .
$$

Then in $H_{2}\left(H^{\mathrm{ab}} / P_{\varphi}\right), a_{1} \wedge a_{2}=\operatorname{det}(\varphi) \cdot a_{3} \wedge a_{4} \neq-a_{3} \wedge a_{4}$; so $P_{\varphi} \in S$. Let

$$
\mathscr{P}_{0}=\left\{P_{\varphi}: \varphi \in T\right\} \cup\left\{P_{0}, P_{\infty}\right\} \subseteq \mathscr{P}
$$

where $P_{0}=\left\{(x, 0): x \in(\mathrm{Z} / p)^{2}\right\}$ and $P_{\infty}=\left\{(0, x): x \in(\mathrm{Z} / p)^{2}\right\}$.

Fix $0 \neq(x, y) \in H^{\mathrm{ab}}$, where $x, y \in(\mathrm{Z} / p)^{2}$. Then $(x, y) \in P_{0}$ if and only if $y$ $=0 ;(x, y) \in P$ if and only if $x=0$. It remains to check that for each $x \neq 0 \neq y$ in $(Z / p)^{2}$, there exists a unique $\varphi \in T$ such that $\varphi(x)=y$. This is most easily seen by identifying $(\mathrm{Z} / p)^{2}$ with $\boldsymbol{F}_{p}[\sqrt{N}] \cong \boldsymbol{F}_{p^{2}}$. Let $x \rightarrow \bar{x}$ be the galois automorphism, and set $N(x)=x \bar{x}$ for $x \in \boldsymbol{F}_{p^{2}}$. Then $T$ consists of all maps $x \rightarrow \alpha x$, for $\alpha \in\left(\boldsymbol{F}_{p^{2}}\right)^{*}$ and $N(\alpha) \neq-1$; and $x \rightarrow \alpha \bar{x}$ for $\alpha \in\left(\boldsymbol{F}_{p^{2}}\right)^{*}$ and $N(\alpha)=-1$, and the result follows.

This procedure fails for the analogous 2-groups: i.e., when $H$ is one of the corresponding central extensions of $\left(C_{2}\right)^{4}$ by $\left(C_{2}\right)^{6}$. So to get an example with 2-groups, we must work instead with a still larger group.

THEOREM 3.5. Define

$$
\begin{gathered}
H=\left\langle a, b, c, d: a^{4}=[b, c d]^{2}, \quad b^{4}=[a, d]^{2}, \quad c^{4}=[b, d]^{2}, \quad d^{4}=[a, c d]^{2},\right. \\
[H,[H, H]]=1\rangle,
\end{gathered}
$$

and set

$$
\tilde{G}=\left\langle H, z:[z, H]=1, z^{8}=[a, b][c, d]\right\rangle .
$$

Let $G=\widetilde{G} / z$. Then the transfer map

$$
\beta^{\#}=\beta_{z}^{\#}: K_{1}(Z G) \rightarrow K_{1}(Z \tilde{G})
$$

for the extension $1 \rightarrow\langle z\rangle \rightarrow \widetilde{G} \stackrel{\beta}{\rightarrow} G \rightarrow 1$ is non-zero.

Proof. Since the proof is somewhat long and involved, we just sketch it in places. Note first that

$$
H^{\mathrm{ab}}=\langle a, b, c, d\rangle \cong\left(C_{4}\right)^{4} ;[H, H]=\langle[a, b],[a, c], \ldots\rangle \cong\left(C_{4}\right)^{6} .
$$

Following the notation of Theorem 3.3, write

$$
q=\exp (H)=8, w=z^{8} \in[H, H], 2^{r}=8 .
$$

Set

$$
S=\left\{\chi: \chi \text { irreducible character of } \tilde{G}, \chi(1)=4, \chi(z)=4 \zeta_{32}\right\} .
$$

In other words, $S$ is the set of all characters for 4-dimensional irreducible 
representations upon which $z$ acts via multiplication with $\zeta_{32}$. Then, in the notation of Theorem 3.3, $n_{\chi}=m_{\chi}=k_{\chi}=2$ for all $\chi \in S$,

$$
F: Z \widetilde{G} \rightarrow \prod_{\chi \in S}(\mathrm{Z} / 4), X=(1)_{\chi \in S} \in \prod_{\chi \in S}(\mathrm{Z} / 4)
$$

and we must show that $X \notin \operatorname{Im}(F)$. Let

$$
x=z^{4} \text { and } \tilde{G}_{0}=\langle H, x\rangle \varsigma \tilde{G} ;
$$

and note that for $\chi \in S$ and $g \in \widetilde{G}-\widetilde{G}_{0}$, all eigenvalues of $g$ acting on $V_{\chi}$ have order at least 16 . In other words, $F(g)=0$ for $g \notin \tilde{G}_{0}$. Thus, if $X \in \operatorname{Im}(F)$, then there exists $\xi \in Z \widetilde{G}_{0}$ such that $F(\xi)=X$. Fix such a $\xi$.

Define projection maps

$$
\alpha: \widetilde{G}_{0} \rightarrow G_{0} /[H, H] \cong\left(C_{4}\right)^{4} \times C_{2} ; \hat{\alpha}: \widetilde{G}_{0} \rightarrow \widetilde{G}_{0} / \operatorname{Fr}(H) \cong\left(C_{2}\right)^{5}
$$

(recall that $\operatorname{Fr}(H)$ is the subgroup generated by commutators and squares in $H$ ). The generators of $\widetilde{G}_{0} /[H, H]$ and $\widetilde{G}_{0} / \operatorname{Fr}(H)$ will also be labelled $a, b, c, d, x$. Set

$$
\begin{aligned}
\mathscr{P}= & \left\{P \subseteq H / \operatorname{Fr}(H) \cong\left(C_{2}\right)^{4}: \operatorname{dim}(P)=2,\right. \\
& P \text { intersects }\langle a, b\rangle \text { or }\langle c, d\rangle, \text { not both }\} .
\end{aligned}
$$

Fix some $P \in \mathscr{P}$, and choose $u, v \in H$ such that $P=\langle\hat{\alpha} u, \hat{\alpha} v\rangle$. Let

$$
K_{P}=\left[H, \hat{\alpha}^{-1} P\right] \subseteq[H, H] .
$$

Then $K_{P}=\left(C_{4}\right)^{5}$, and (by the intersection assumption on $P$ ):

$$
\left\langle K_{P},[a, b][c, d]=x^{2}\right\rangle=[H, H] .
$$

Fix $i, j \in\{0,1\}$ such that $u x^{i}, v x^{j}$ have order 4 in $\tilde{G}_{0} / K_{P}$.

Let $\zeta=\zeta_{8}=\left(\zeta_{32}\right)^{4}$. There exist irreducible $\tilde{G}$-representations $V(u, v)$ and $V^{\prime}(u, v)$, whose characters $\chi(u, v)$ and $\chi^{\prime}(u, v)$ lie in $S$, and such that

(i) on both $V$ and $V^{\prime}$, elements of $K_{P}$ act trivially, and $x$ acts via multiplication by $\zeta$,

(ii) on $V(u, v), u x^{i}$, and $v x^{j}$ act via the identity,

(iii) in $V^{\prime}(u, v), u x^{i}$, and $v x^{j}$ act vis multiplication by $\zeta^{2}$,

(iv) for any $g \in \widetilde{G}_{0}$ such that $\hat{\alpha}(g) \notin\langle P, x\rangle=\langle\hat{\alpha} u, \hat{\alpha} v, x\rangle, g$ has eigenvalues (on $V$ and $V^{\prime}$ ): $\left\{1, \zeta^{2},-1,-\zeta^{2}\right\}$ or $\left\{\zeta, \zeta^{3}, \zeta^{5}, \zeta^{7}\right\} ; g^{2}$ has eigenvalues $\{1,1,-1$, $-1\}$ or $\left\{\zeta^{2}, \zeta^{2},-\zeta^{2},-\zeta^{2}\right\}$.

By (iv), for any $g \in H$,

$$
\begin{array}{rlrl}
F_{V(u, v)}(g+g x) & =\operatorname{dim}\left(V(u, v)^{g}\right)+\operatorname{dim}\left(V(u, v)^{g x}\right. \\
& =1 & & \text { if } \hat{\alpha}(g) \notin P \\
& \equiv 0(\bmod 2) & \text { if } \hat{\alpha}(g) \in P .
\end{array}
$$


Also, if $g \in \operatorname{Fr}(H)$ (that is, $\hat{\alpha}(g)=1$ ), then

$$
F_{V(u, v)}(g) \equiv F_{V(u, v)}(g x) \equiv 0(\bmod 2) .
$$

Furthermore, by (iv), we get, for any $g \in \widetilde{G}_{0}$

$$
\begin{gathered}
F_{V(u, v)}(g)-F_{V^{\prime}(u, v)}(g)=2 \text { if } g \equiv u x^{i} \text { or } v x^{j}(\bmod \operatorname{Fr}(H)) \\
\quad \text { but } g \notin\langle u, v,[H, H], x\rangle \\
=0 \text { otherwise. }
\end{gathered}
$$

Hence, if $t_{1}, t_{2}, t_{3}, t_{4}$ are coset representatives for $P \subseteq H / \operatorname{Fr}(H)$, then for any $g \in \widetilde{G}_{0}$,

$$
\begin{aligned}
\sum_{i=1}^{4}\left[F_{V\left(t_{i}^{2} u, t_{i}^{2} v\right)}-F_{V^{\prime}\left(t_{i}^{2} u, t_{i}^{2} v\right)}\right](g) & \equiv 2 \text { if } g \equiv u x^{i} \text { or } v x^{j}(\bmod \operatorname{Fr}(H)) \\
& \equiv 0(\bmod 4) \text { otherwise. }
\end{aligned}
$$

Hence, if $F(\xi)=X$, the coefficients of $u x^{i}$ and $v x^{j}$ in

$$
\hat{\alpha}(\xi) \in \mathbf{Z}\left[\tilde{G}_{0} / \operatorname{Fr}(H)\right]
$$

are congruent modulo 2. The same argument holds for the pair $\left\{u x^{i}, u v x^{i+j}\right\}$ (note that $\left.\left(u v x^{i+j}\right)^{4} \in K_{P}\right)$; so all three of $\left\{u x^{i}, v x^{j}, u v x^{i+j}\right\}$ have the same coefficient $(\bmod 2)$ in $\hat{\alpha}(\xi)$.

Direct computations now give the following list of such triples, one for each subplane $P \in \mathscr{P}$ :

$\begin{array}{cccccc}a & b & a b & c & d & c d \\ a x & b c x & a b c & c x & a d x & a c d \\ a x & b d & a b d x & c & b d & b c d \\ a & b c d & a b c d & c x & a b d & a b c d x \\ b x & a c & a b c x & d & a c x & a c d x \\ b & a d x & a b d x & d x & b c & b c d x \\ b x & a c d x & a b c d & d x & a b c & a b c d x \\ a b & a c & b c & c d x & a c x & a d \\ a b x & a d & b d x & c d & b c x & b d x \\ a b x & a c d & b c d x & c d x & a b c x & a b d\end{array}$.

By inspection, the equivalence relation on $\left(\widetilde{G}_{0} / \operatorname{Fr}(H)-\{1, x\}\right)$, generated by lying in the same triple, is transitive.

Hence, for some $r, s, t \in Z$,

$$
\hat{\alpha}(\xi) \equiv r+s x+t \sum_{\substack{g \in H / F^{\prime}(H) \\ g \neq 1}}(g+g x)(\bmod 2) .
$$

So by (1) and (2) above, if $V=V(u, v)$ for any $u, v$, then 


$$
F_{V}(\xi) \equiv t \cdot \sum_{\substack{g \in H / F_{\mathrm{r}}(H) \\ g \neq 1}} F_{V}(g+g x) \equiv 0(\bmod 2)
$$

since $(H / \operatorname{Fr}(H)-P)$ has even order. But $X_{V}=1$, contradicting the assumption that $F(\xi)=X$. It follows that $X \notin \operatorname{Im}(F)$; and hence that the transfer map $\beta^{\#}$ is non-zero.

4.

We now study the Whitehead transfer homomorphism for an arbitrary central extension of finite groups with cyclic kernel. The main results are collected in Theorems 4.9, 4.10, and 4.11.

Throughout this section, $p$ denotes a fixed prime. For any finite group $G$, $G_{r}$ and $G_{p}$ will denote the sets of $p$-regular elements and $p$-elements of $G$,

$$
G_{r}=\{g \in G: p \nmid|g|\} \text { and } G_{p}=\left\{g \in G:|g|=p^{i} \text {, some } i\right\} \text {. }
$$

Then any $g \in G$ can be written uniquely as a product $g=g_{r} g_{p}$, such that $g_{r} \in G_{r}, g_{p} \in G_{p}$, and $g_{p} g_{r}=g_{r} g_{p}$. Two elements $g, g^{\prime} \in G$ will be called $p^{\prime}-$ conjugate in $G$ if for some $g^{\prime \prime}$ conjugate to $g^{\prime}, g_{p}=g_{p}^{\prime \prime}$ and $\left\langle g_{r}\right\rangle=\left\langle g_{r}^{\prime \prime}\right\rangle$.

Let $\hat{Z}_{p}\left(G_{r}\right)$ denote the free $\hat{Z}_{p}$-module with basis $G_{r}$, and with the action of $G$ induced by conjugation. Define

$$
\Phi: \hat{Q}_{p} G \rightarrow \hat{Q}_{p} G, \Phi: \hat{Z}_{p}\left(G_{r}\right) \rightarrow \hat{Z}_{p}\left(G_{r}\right)
$$

by setting $\Phi\left(\sum \lambda_{i} g_{i}\right)=\sum \lambda_{i} g_{i}^{p}\left(\lambda_{i} \in \hat{Q}_{p}, g_{i} \in G\right)$. Set

$$
\begin{aligned}
& H_{1}\left(G ; \hat{Z}_{p}\left(G_{r}\right)\right)_{\Phi}=H_{1}\left(G ; \hat{Z}_{p}\left(G_{r}\right)\right) /(1-\Phi) \\
& =H_{1}\left(G ; \hat{Z}_{p}\left(G_{r}\right)\right) /\left\langle g \otimes\left(h-h^{p}\right): h \in G_{r}, g h=h g\right\rangle
\end{aligned}
$$

and

$$
H_{0}\left(G ; Z / p\left(G_{r}\right)\right)_{\Phi}=H_{0}\left(G ; Z / p\left(G_{r}\right)\right) /(1-\Phi)=H_{0}\left(G ; Z / p\left(G_{r}\right)\right) /\left\langle h-h^{p}\right\rangle .
$$

Homomorphisms

$$
\begin{gathered}
\Gamma=\Gamma_{G}: K_{1}\left(\hat{Z}_{p} G\right) \rightarrow H_{0}\left(G ; \hat{\mathrm{Q}}_{p} G\right) \text {, and } \\
(\bar{\omega}, \bar{\theta})=\left(\bar{\omega}_{G}, \bar{\theta}_{G}\right): H_{0}\left(G ; \hat{Z}_{p} G\right) \rightarrow H_{1}\left(G ; \hat{Z}_{p}\left(G_{r}\right)\right)_{\Phi} \oplus H_{0}\left(G ; Z / p\left(G_{r}\right)\right)_{\Phi}
\end{gathered}
$$

can now be defined. As in the $p$-group case,

$$
\Gamma_{G}(u)=\log (u)-1 / p \Phi(\log (u))\left(\text { any } u \in K_{1}\left(\hat{Z}_{p} G\right)\right) .
$$

For any $\lambda_{i} \in \vec{Z}_{p}$ and $g_{i} \in G$, with $\bar{\lambda}_{i} \in \mathbf{Z} / p$ the reduction of $\lambda_{i}$, set

$$
(\bar{\omega}, \bar{\theta})\left(\sum \lambda_{i} g_{i}\right)=\left(\sum g_{i} \otimes \lambda_{i}\left(g_{i}\right)_{r}, \sum \bar{\lambda}_{i}\left(g_{i}\right)_{r}\right)
$$


$\left(\left(g_{i}\right)_{r}\right.$ denotes the $p$-regular part of $g_{i}$, as described above).

THEOREM 4.1. For any finite group $G, \Gamma_{G}$ is a well defined homomorphism, and $\operatorname{Im}\left(\Gamma_{G}\right) \subseteq H_{0}\left(G ; \hat{Z}_{p} G\right)$. Furthermore,

(i) If $p$ is odd, then

$$
\operatorname{Im}\left(\Gamma_{G}\right)=\operatorname{Ker}\left[\bar{\omega}_{G}: H_{0}\left(G ; \hat{Z}_{p} G\right) \rightarrow H_{1}\left(G ; \hat{Z}_{p}\left(G_{r}\right)\right)_{\Phi}\right] ;
$$

while if $p=2$

$$
\begin{gathered}
\operatorname{Im}\left(\Gamma_{G}\right)=\operatorname{Ker}\left[\left(\bar{\omega}_{G}, \bar{\theta}_{G}\right): H_{0}\left(G ; \hat{Z}_{2} G\right) \rightarrow H_{1}\left(G ; \hat{Z}_{2} G_{r}\right)\right)_{\Phi} \\
\left.\times H_{0}\left(G ; \mathrm{Z} / 2\left(G_{r}\right)\right)_{\Phi}\right] .
\end{gathered}
$$

(ii) For any central $z \in G$ of p-power order,

$$
\begin{gathered}
\Gamma_{G}\left(1+(1-z) \hat{Z}_{p} G\right)=\left\langle(1-z)\left(g-g^{p}\right): g \in G\right\rangle \\
=\left\langle(1-z)(a-1) h: a \in G_{p}, h \in G_{r}, a h=h a\right\rangle+\left\langle(1-z)\left(h-h^{p}\right): H \in G_{r}\right\rangle .
\end{gathered}
$$

(iii) If $i_{*}: K_{1}(\mathrm{ZG}) \rightarrow K_{1}\left(\hat{\mathrm{Z}}_{p} G\right)$ is induced by inclusion, then

$$
\begin{aligned}
\Gamma_{G}\left(i_{*}\left(K_{1}(Z G)\right) \subseteq\right. & \left\langle g+g^{-1}-g^{m}-g^{-m}:(m,|g|)=1\right\rangle \\
& +\left\langle g-g^{m}:(m,|g|)=1, g \text { conj } g^{-1}\right\rangle .
\end{aligned}
$$

Proof. That the logarithm (and hence $\Gamma_{G}$ ) are well defined is shown in [22, Theorem 1.1]. The image of $\Gamma_{G}$ is described in [22, Corollary 1.8].

(ii) Note first that for any $i \geqq 1,1+(1-z)^{i} \hat{Z}_{p} G$ is generated by $1+(1-z)^{i+1} \hat{Z}_{p} G$ together with elements $1+(1-z)^{i} g$, for $g \in G$. Since $(1-z)^{i} \rightarrow 0$ as $i \rightarrow \infty$ in the $p$-adic topology $\left(p \mid(1-z)^{i}\right.$ if $\left.i \geqq|z|\right)$, this shows that the subgroup generated by all such $1+(1-z)^{i} g$ is dense in $1+(1-z) \hat{Z}_{p} G$.

For any $i \geqq 1$,

$$
\begin{aligned}
\left(1-z^{p}\right)^{i} & =\left(1-[1-(1-z)]^{p}\right)^{i} \equiv\left(p(1-z)-(-1)^{p}(1-z)^{p}\right)^{i} \\
& \equiv\left(p(1-z)+(1-z)^{p}\right)^{i} \equiv p^{i}(1-z)^{i}+(1-z)^{p i}\left(\bmod p(1-z)^{i+1}\right) .
\end{aligned}
$$

Hence, for any $i \geqq 1$ and any $j \geqq 0$,

$$
\begin{aligned}
& \left(1+p^{j}(1-z)^{i} g\right)^{p} /\left(1+p^{j}\left(1-z^{p}\right)^{i} g^{p}\right) \\
& \quad \equiv 1+p^{j+1}(1-z)^{i} g+p^{p j}(1-z)^{p i} g^{p}-p^{j}\left(1-z^{p}\right)^{i} g^{p}\left(\bmod p(1-z)^{i+1}\right) \\
& \quad \equiv 1+p^{j+1}(1-z)^{i} g-p^{j+i}(1-z)^{i} g^{p}+\left(p^{p j}-p^{j}\right)(1-z)^{p i} g^{p} \\
& \quad \equiv 1+p^{j+1}(1-z)^{i}\left(g-p^{i-1} g^{p}\right) .
\end{aligned}
$$

It follows that 


$$
\begin{gathered}
\Gamma\left(1+p^{j}(1-z)^{i} g\right)=1 / p \log \left[\left(1+p^{j}(1-z)^{i} g\right)^{p} /\left(1+p^{j}\left(1-z^{p}\right)^{i} g^{p}\right)\right] \\
\equiv p^{j}(1-z)^{i}\left(g-p^{i-1} g^{p}\right) \quad\left(\bmod (1-z)^{i+1} \hat{Z}_{p} G\right)
\end{gathered}
$$

for any $i \geqq 1$ and $j \geqq 0$; and hence that

$$
\Gamma_{G}\left(1+(1-z) \hat{Z}_{p} G\right)=\left\langle(1-z)\left(g-g^{p}\right): g \in G\right\rangle+\left\langle(1-z)^{i} g: g \in G, i \geqq 2\right\rangle .
$$

In particular, this shows that as subgroups of $H_{0}\left(G ; \hat{Z}_{p} G\right)$,

$$
\begin{aligned}
\langle(1-z)( & \left.\left(g-g^{p}\right): g \in G\right\rangle \subseteq \Gamma_{G}\left(1+(1-z) \hat{Z}_{p} G\right) \\
\subseteq & \left\langle(1-z)(a-1) h: a \in G_{p}, h \in G_{r}, a h=h a\right\rangle+ \\
& +\left\langle(1-z)\left(h-h^{p}\right): h \in G_{r}\right\rangle .
\end{aligned}
$$

For any commuting pair of elements $a \in G_{p}$ and $h \in G_{r},(a h)^{p^{n}}=h$ for some $n \geqq 1$; so the subgroups in (1) are all equal, and this proves (ii).

(iii) Assume first $G$ is cyclic, and consider the inclusion

$$
\mathrm{Z} G \hookrightarrow \mathrm{Q} G \cong \prod_{d \mid n} \mathrm{Q} \zeta_{d}
$$

(where $n=|G|$ ). For any unit $u \in\left(Z \zeta_{d}\right)^{*}, \bar{u} / u$ is a root of unity, and $N(u) \in Z^{*}=\{ \pm 1\}$. Hence, for any $u \in K_{1}(Z G)$,

$$
c_{*}(u) / u \text { and } \prod_{\eta \in \operatorname{Aut}(G)} \eta_{*}(u)
$$

are both torsion $\left(c \in \operatorname{Aut}(G)\right.$ denotes the map: $\left.c(g)=g^{-1}\right)$. Thus,

$$
c_{*}\left(\Gamma_{G}\left(i_{*}(u)\right)\right)=\Gamma_{G}\left(i_{*}(u)\right) \text { and } \sum_{\eta \in \operatorname{Aut}(G)} \eta_{*}\left(\Gamma_{G}\left(i_{*}(u)\right)\right)=0,
$$

and hence

$$
\Gamma_{G}\left(i_{*}(u)\right) \in\left\langle g+g^{-1}-g^{m}-g^{-m}:(m,|g|)=1\right\rangle .
$$

Now let $G$ be arbitrary. By [14, Theorems 3.3.4 and 4.2.1], for any $u \in K_{1}(Z G)$,

$$
u^{k} \in \operatorname{Im}\left[\sum\left\{K_{1}(Z H): H \subseteq G \text { cyclic }\right\} \stackrel{\text { Ind }}{\longrightarrow} K_{1}(Z G)\right]
$$

for some $k \geqq 1$. Thus,

$$
\begin{aligned}
& \Gamma_{G}\left(i_{*}\left(K_{1}(Z G)\right)\right) \\
& \quad \subseteq H_{0}\left(G ; \hat{Z}_{p} G\right) \cap\left\langle g+g^{-1}-g^{m}-g^{-m}: g \in G,(m,|g|)=1\right\rangle_{Q_{p}} \\
& \quad=\left\langle g+g^{-1}-g^{m}-g^{-m}:(m,|g|)=1\right\rangle+\left\langle g-g^{m}:(m,|g|)=1, g \text { conj } g^{-1}\right\rangle .
\end{aligned}
$$


The next lemma is quite technical, but it is the key to getting a limit on the size of the $\operatorname{Im}\left(\beta_{z}^{\#}\right)$.

LeMMA 4.2. Let $\pi$ be a p-group, fix elements $z \in Z(\pi)$ and $g \in \pi$, and set $\sigma=\langle g\rangle$. Write $\hat{\mathrm{Q}}_{p}[\pi]=\hat{\mathrm{Q}}_{p}[\pi / z] \times R$, and define, for any unit $u \in\left(\hat{\mathrm{Z}}_{p}[\sigma]\right)^{*}$,

$$
\widetilde{E}_{z}(u)=(1,\{1-z, u\}) \in K_{2}^{c}\left(\hat{\mathrm{Q}}_{p}[\pi / z]\right) \times K_{2}^{c}(R)=K_{2}^{c}\left(\hat{\mathrm{Q}}_{p}[\pi]\right) .
$$

(i) Assume that $\Gamma_{\sigma}(u)=g+g^{-1}-g^{m}-g^{-m}$ for some $p \nmid m$. Then $\widetilde{E}_{z}(u)=1$.

(ii) Assume that $p=2$, and that there is an automorphism $\eta$ of $\pi$ such that $\eta(z)=z$, and $\eta(g)=z^{t} g^{-1}$ for some $t$. If

$$
\Gamma_{\sigma}(u)=\sum \lambda_{i} g^{i},
$$

where $\sum \lambda_{i}=0$ and $\lambda_{i}=0$ for even $i$, then

$$
\widetilde{E}_{z}(u) \in \operatorname{Im}\left[K_{2}^{c}\left(\hat{Z}_{2} \pi\right) \rightarrow K_{2}^{c}\left(\hat{Q}_{2} \pi\right)\right]+\left\{\left(1-\eta_{*}\right)(x): x \in K_{2}^{c}\left(\hat{Q}_{2} \pi\right)\right\} .
$$

Proof. By naturality, it suffices to prove this when $\pi=\langle z\rangle \times\langle g\rangle$, and $|z|=|g|=p^{n}$ for some $n$. For any $k \geqq 0$, and any $r, s \in Z$, let

$$
\chi_{k}(r, s): \mathrm{Q} \pi \rightarrow \mathrm{Q} \zeta_{p^{k}}
$$

be the ring homomorphism defined by:

$$
\chi_{k}\left(r, s^{\dagger}\right)(z)=\left(\zeta_{p^{k}}\right)^{r} ; \chi_{k}(r, s)(g)=\left(\zeta_{p^{k}}\right)^{s} .
$$

Let

$$
\begin{aligned}
& S=\left\{\chi_{k}(1, s): 0 \leqq k \leqq n, 0 \leqq s \leqq p^{k}-1\right\} \\
& \cup\left\{\chi_{k}(r, 1): 1 \leqq k \leqq n, 0 \leqq r \leqq p^{k}-1, p \mid r\right\} .
\end{aligned}
$$

Then the map

$$
\prod \chi: \mathrm{Q} \pi \rightarrow \prod_{\chi \in S} \mathrm{Q}(\chi) \quad(\mathrm{Q}(\chi)=\mathrm{Q}(\operatorname{Im}(\chi)))
$$

is an isomorphism.

We will have use for the following homomorphisms, defined for $k \leqq n$ and $r, s \in \mathrm{Z}$ :

$(\cdot, \cdot)_{k}: K_{2}^{c}\left(\hat{Q}_{p} \zeta_{p^{k}}\right) \rightarrow\left\langle\zeta_{p^{k}}\right\rangle$ denotes the norm residue symbol $\chi_{k}(r, s)_{*}: K_{2}^{c}\left(\hat{Q}_{p} \pi\right) \rightarrow\left\langle\zeta_{p^{k}}\right\rangle$ denotes the composite $(\cdot, \cdot)_{k}^{\circ} \chi_{k}(r, s)$ $\psi_{k}(r): \hat{Q}_{p} \sigma \rightarrow\left\langle\zeta_{p^{n}}\right\rangle(\sigma=\langle g\rangle)$ is defined by $\psi_{k}(r)(g)=\left(\zeta_{p^{k}}\right)^{r}$.

(i) Fix $p \nmid m$, and assume that $\Gamma_{\sigma}(u)=g+g^{-1}-g^{m}-g^{-m}$. Fix $k \leqq n$, and set $\zeta=\zeta_{p^{k}}$. For any $0 \leqq s<p^{k}$, 


$$
\chi_{k}(1, s)_{*}\left(\widetilde{E}_{z}(u)\right)=\left(1-\zeta, \psi_{k}(s)(u)\right)_{k}=\zeta^{R},
$$

where by Proposition 2.9,

$$
R=\left[s / p^{k}\right]+\left[-s / p^{k}\right]-\left[m s / p^{k}\right]-\left[-m s / p^{k}\right] .
$$

Since $p \nmid m, s / p^{k} \in Z$ if and only if $m s / p^{k} \in Z$, and hence $R=0$. So

$$
\chi_{k}(1, s)_{*}\left(\tilde{E}_{z}(u)\right)=1 \text {. }
$$

For any $0<r<p^{k}$ such that $p \mid r$,

$$
\chi_{k}(r, 1)_{*}\left(\widetilde{E}_{z}(u)\right)=\left(1-\zeta^{r}, \psi_{k}(1)(u)\right)_{k} .
$$

Write $r=r^{\prime} p^{a}$, where $p \nmid r^{\prime}$; then

$$
1-\zeta^{r}=1-\zeta^{r^{\prime} p^{a}}=\prod_{i=0}^{p^{a}-1}\left(1-\zeta^{r^{\prime}+i p^{k-a}}\right)
$$

Each $\zeta^{r^{\prime}+i p^{k-a}}$ is a primitive $p^{a}$ th root of unity $(a<k)$, and so

$$
\left(1-\zeta^{r}, \psi_{k}(1)(u)\right)_{k}=\prod_{i=0}^{p^{a}-1}\left(1-\zeta^{r^{\prime}+i p^{k-a}}, \chi_{k}(1)(u)\right)_{k}=1
$$

by the same calculation as above. Finally, $\chi_{k}(0,1)_{*}\left(\widetilde{E}_{z}(u)\right)=1$ by definition, and thus $\widetilde{E}_{z}(u)=1$ in $K_{2}^{c}\left(\widehat{Q}_{p} \pi\right)$.

(ii) Now $p=2$. For each $i, j$ such that $i=1$, or $2 \mid i$ and $j=1$, let $x_{i j} \in K_{2}^{c}\left(\hat{Q}_{2} \pi\right)$ be the element such that for all $\chi_{k}(r, s) \in S$,

$$
\begin{aligned}
\chi_{k}(r, s)_{*}\left(x_{i j}\right) & =\zeta_{2^{k}} & & \text { if } 0 \leqq k \leqq n, r \equiv i, s \equiv j\left(\bmod 2^{k}\right) \\
& =1 & & \text { otherwise. }
\end{aligned}
$$

Define a subgroup $V \subseteq K_{2}^{c}\left(\hat{Q}_{2} \pi\right)$ by setting

$$
V=\left\langle x_{1 j}-x_{1, j+2}, 2 x_{1 j}, x_{i 1}-x_{i+2,1}, 2 x_{i 1}: j \in Z, i \in 2 Z\right\rangle \text {. }
$$

Then

$$
V=\left\{x \in K_{2}^{c}\left(\hat{Q}_{2} \pi\right) \text { : conditions (a) and (b) below hold for } x\right\},
$$

where

(a) for any $1 \leqq k \leqq n$, any $s \in Z$, and $r \in 2 Z$ :

$$
\begin{aligned}
& \chi_{k-1}(1, s)_{*}(x)=\left[\chi_{k}(1, s)_{*}(x) \cdot \chi_{k}\left(1, s+2^{k-1}\right)_{*}(x)\right]^{2} \\
& \chi_{k-1}(r, 1)_{*}(x)=\left[\chi_{k}(r, 1)_{*}(x) \cdot \chi_{k}\left(r+2^{k-1}, 1\right)_{*}(x)\right]^{2}
\end{aligned}
$$

(b) $\chi_{1}(r, s)_{*}(x)=1$ for all $(r, s)=(1,0),(1,1),(0,1)$. 


$$
W=\left\{x \in K_{2}^{c}\left(\hat{\mathrm{Q}}_{2} \pi\right): \chi_{*}(x)=1 \text { for } \chi \in S \text { such that } \chi(z) \neq 1\right) .
$$

In other words, $W$ is the image of $K_{2}^{c}\left(\hat{Q}_{2}[\pi / z]\right)$ under the canonical splitting map. Since $\eta \in$ Aut $(\pi)$ is such that $\eta(z)=z$ and $\eta(g)=z^{t} g^{-1}$ for some $t, \eta$ acts on $W$ by sending elements to their inverses. Thus, $W^{2} \subseteq\left(\eta_{*}-1\right) K_{2}^{c}\left(\hat{Q}_{2} \pi\right)$. Part (ii) will follow upon showing that

$$
\begin{aligned}
& \text { (I) } V \subseteq \operatorname{Im}\left[K_{2}^{c}\left(\hat{\mathrm{Z}}_{2} \pi\right) \rightarrow K_{2}^{c}\left(\hat{\mathrm{Q}}_{2} \pi\right)\right] \text {, and } \\
& \text { (II) } \widetilde{E}_{z}(u) \in\left\langle V, W^{2}\right\rangle \text {. }
\end{aligned}
$$

STEP I. Fix $j \in Z$, and define $v_{1}, v_{2} \in\left(\hat{Z}_{2} \pi\right)^{*}$ so that

$$
\Gamma_{\pi}\left(v_{1}\right)=z^{-j} g-z^{-j-2} g+\left(z-z^{3}\right) ; \Gamma_{\pi}\left(v_{2}\right)=z^{-j} g+z^{j} g^{-1}
$$

(see Theorem 2.7). Furthermore, upon multiplying by some element of $\langle-1, z, g\rangle$, if necessary, we may assume that

$$
v_{1}, v_{2} \in \operatorname{Ker}\left[\left(\hat{Z}_{2} \pi\right)^{*} \rightarrow\left(\hat{Z}_{2}\left[\pi /\left\langle g^{2}, z^{2}\right\rangle\right]\right)^{*}\right] .
$$

For any $\chi=\chi_{k}(r, s) \in S, \chi(z)=1$ if and only if $\chi\left(z^{3}\right)=1, \chi\left(z^{-j} g\right)=1$ if and only if $r \equiv 1$ and $s \equiv j\left(\bmod 2^{k}\right)$, and similarly for $z^{-j-2} g$ and $z^{j} g^{-1}$. So Proposition 2.9 (ii) applies to show that

$$
x_{1 j}-x_{1, j+2}=\left\{-z, v_{1}\right\} \text { and } 2 x_{1 j}=\left\{-z, v_{2}\right\} \text {, }
$$

and hence that both lie in $\operatorname{Im}\left[K_{2}^{c}\left(\hat{Z}_{2} \pi\right) \rightarrow K_{2}^{c}\left(\hat{Q}_{2} \pi\right)\right]$. Note that (3) insures that $\chi_{1}(r, s)_{*}\left(\left\{-z, v_{1}\right\}\right) \doteq 1$ for all $r, s$. By symmetry, the same result holds for the $x_{i 1}-x_{i+2,1}$ and $2 x_{i 1}$; and this proves (I).

STEP II. By hypothesis, $\Gamma_{\sigma}(u)=\sum_{i=0}^{2^{n}-1} \lambda_{i} g^{i}$, where $\lambda_{i}=0$ for even $i$, and $\sum \lambda_{i}=0$. For each $1 \leqq k \leqq n$ and $r \in Z$, Theorem 2.7 (iii) shows (where we set $\left.\zeta=\zeta_{2^{k}}\right)$,

$$
\begin{gathered}
\log \left(\chi_{k}(r)(u)\right)=\sum_{\substack{i=0 \\
2 \nmid i}}^{2^{n}-1} \lambda_{i}\left[\left(\zeta^{i r}-1\right)+\frac{1}{2}\left(\zeta^{2 i r}-1\right)+\frac{1}{4}\left(\zeta^{4 i r}-1\right)+\ldots\right], \\
\log \left(\chi_{k}\left(r+2^{k-1}\right)(u)\right)=\sum_{\substack{i=0 \\
2 \nmid i}}^{2^{n}-1} \lambda_{i}\left[\left(-\zeta^{i r}-1\right)+\frac{1}{2}\left(\zeta^{2 i r}-1\right)+\frac{1}{4}\left(\zeta^{4 i r}-1\right)+\ldots\right],
\end{gathered}
$$

and (recall again that $\sum \lambda_{i}=0$ ),

$$
\begin{aligned}
\log \left(\chi_{k-1}(r)(u)\right) & =\sum_{\substack{i=0 \\
2 \times i}}^{2^{n}-1} \lambda_{i}\left[\left(\zeta^{2 i r}-1\right)+\frac{1}{2}\left(\zeta^{4 i r}-1\right)+\ldots\right] \\
& =\log \left(\chi_{k}(r)(u)\right)+\log \left(\chi_{k}\left(r+2^{k-1}\right)(u)\right) .
\end{aligned}
$$


It follows that

$$
\chi_{k-1}(r)(u) \equiv \chi_{k}(r)(u) \cdot \chi_{k}\left(r+2^{k-1}\right)(u)\left(\bmod \left\langle\zeta_{2^{k}}\right\rangle\right)
$$

Note also that for any $k$ and $r$,

$$
\left(1-\left(\zeta_{2^{k}}\right)^{r}, \zeta_{2^{k}}\right)_{k}=1
$$

by (1) and the fact that $\left(1-\zeta_{2^{k}}, \zeta_{2^{k}}\right)_{k}=1$.

Now, for any $k \leqq n$ and any $r, s$, we write

$$
X=\tilde{E}_{z}(u) \text { and } X_{k}(r, s)=\chi_{k}(r, s)_{*}\left(\tilde{E}_{z}(u)\right)
$$

for short. Then, for any $1 \leqq k \leqq n$ and any $s$,

$$
\begin{aligned}
X_{k-1} & (1, s)=\left(1-\zeta^{2}, \chi_{k-1}(s)(u)\right)_{k-1} & & \left(\zeta=\zeta_{2^{k}}\right) \\
& =\left(N(1-\zeta), \chi_{k-1}(s)(u)\right)_{k-1}=\left[\left(1-\zeta, \chi_{k-1}(s)(u)\right)_{k}\right]^{2} & & (\text { Thm. 2.4) } \\
(6) & =\left[\left(1-\zeta, \chi_{k}(s)(u)\right)_{k} \cdot\left(1-\zeta, \chi_{k}\left(s+2^{k-1}\right)(u)\right)_{k}\right]^{2} & & (\text { by (4), (5)) } \\
& =\left(X_{k}(1, s) \cdot X_{k}\left(1, s+2^{k-1}\right)\right)^{2} . & &
\end{aligned}
$$

Similarly, for any $k$ and any even $2 \leqq r \leqq 2^{k-1}-2$,

$$
X_{k-1}(r, 1)=\left(X_{k}(r, 1) \cdot X_{k}\left(r+2^{k-1}, 1\right)\right)^{2} .
$$

In addition, the conditions on $\Gamma_{\sigma}(u)$ show that the image of $u$ in $\hat{Z}_{2}\left[\sigma / g^{2}\right]$ has finite order; and hence (using (5) again) that

$$
X_{1}(r, s)=1 \text { for }(r, s)=(1,0),(1,1),(0,1) .
$$

Now let $Y \in K_{2}^{c}\left(\hat{Q}_{2} \pi\right)$ be the element defined by setting

$$
\begin{array}{ll}
\chi_{k}(0,1)_{*}(Y)=\prod_{l=k+1}^{n}\left(X_{l}\left(2^{l-1}, 1\right)\right)^{2^{l-k}} & \text { all } 0 \leqq k \leqq n \\
\chi_{k}(r, s)_{*}(Y)=1 & \text { if } \chi_{k}(r, s) \in S \text { and }(r, s) \neq(0,1) .
\end{array}
$$

Recall that $\chi_{k}(0,1)_{*}(X)=1$ by definition. So for $1 \leqq k \leqq n$,

$$
\chi_{k-1}(0,1)_{*}(X Y)=\left[\chi_{k}(0,1)_{*}(X Y)\right]^{2} \cdot\left[\chi_{k}\left(2^{k-1}, 1\right)_{*}(X Y)\right]^{2} ;
$$

and this together with (6) and (7) shows that condition (a) holds for $X Y$. Furthermore, for any $k$,

$$
\begin{aligned}
\left(X_{k}\left(2^{k-1}, 1\right)\right)^{2^{k-1}} & =\left\{\left(2, \chi_{k}(1)(u)\right)_{k}\right]^{2^{k-1}}=\left(2, N_{Q \zeta_{2^{k}} / \mathrm{Q}}\left(\chi_{k}(1)(u)\right)\right)_{1} \\
& =\left(2, \prod_{i=1}^{2^{k}-1} \chi_{k}(i)(u)\right)_{1}=\left(2, \chi_{1}(1)(u)\right)_{1}(\text { by }(4) \text { and }(5)) \\
& =(2, \pm 1)_{1}=1 .
\end{aligned}
$$


In particular, this shows that $\chi_{1}(0,1)_{*}(Y)=1$, and so (using also (8)), condition (b) also holds for $X Y$. Thus, $X Y \in V$. Finally, $Y \in W^{2}$ by (9) (and the definition of $Y$ ); and so

$$
X=\tilde{E}_{z}(u) \in\left\langle V, W^{2}\right\rangle .
$$

Now fix a central extension

$$
1 \rightarrow \sigma \rightarrow \widetilde{G} \stackrel{\beta}{\longrightarrow} G \rightarrow 1
$$

of finite groups, such that $\sigma \subseteq Z(\widetilde{G})$ has $p$-power order. It will be seen later how the study of the transfer in general is reduced to this case. The following diagram, defined for any generator $z$ of $\sigma=\operatorname{Ker}(\beta)$, will be referred to frequently in the next several lemmas and propositions (note that $\operatorname{Im}\left(\beta_{z}^{\#}\right)$ is a $p$-group, by Proposition 1.4):

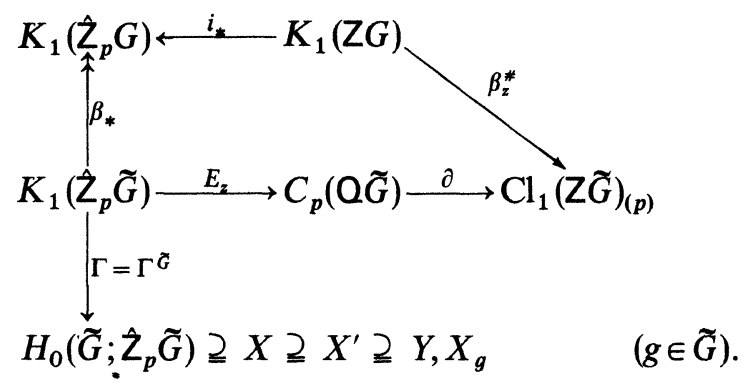

These homomorphisms and subgroups are defined as follows:

(i) $\partial$ is the boundary map in the localization exact sequence of Theorem 2.1 , and $i_{*}$ and $\beta_{*}$ are induced by the obvious ring homomorphisms. Note that $\beta_{*}$ is induced by a surjection of semilocal rings, and is hence onto [8, Corollary III. 2.9].

(ii) Write $Q \tilde{G}=Q G \times R$; then

$$
E_{z}(u)=(1,\{1-z, u\}) \in C_{p}(Q G) \times C_{p}(R)=C_{p}(Q \tilde{G})
$$

for any $u \in K_{1}\left(\hat{Z}_{p} \tilde{G}\right)$ and any $z$ generating $\operatorname{Ker}(\beta)$.

(iii)

$$
\begin{aligned}
Y & =\left\{\sum_{i=1}^{k} \lambda_{i}\left(g_{i}-g_{i}^{\prime}\right): \lambda_{i} \in \vec{Z}_{p}, g_{i} p^{\prime} \text {-conjugate to } g_{i}^{\prime} \text {, all } i\right\} \\
& =\left\langle a\left(h-h^{m}\right): a \in \tilde{G}_{p}, h \in \tilde{G}_{r}, a h=h a,(m,|h|)=1\right\rangle \subseteq H_{0}\left(\tilde{G} ; \vec{Z}_{p} \tilde{G}\right) .
\end{aligned}
$$

(iv) For any $g \in \tilde{G}$

$$
X_{g}=\left\langle g+g^{-1}-g^{m}-g^{-m}:(m,|g|)=1\right\rangle
$$


if $p$ is odd, or if $\beta(g)$ is not $p^{\prime}$-conjugate to $\beta\left(g^{-1}\right)$ in $G$; and

$$
X_{g}=\left\langle\sum_{i=0}^{k} \lambda_{i} g^{i}: \lambda_{i}=0 \text { if }(i,|g|) \neq 1, \sum \lambda_{i}=0, \sum i \lambda_{i} \equiv 0(\bmod |g|)\right\rangle
$$

if $p=2$ and $\beta(g)$ is $p^{\prime}$-conjugate to $\beta\left(g^{-1}\right)$ in $G$.

(v)

$$
\begin{aligned}
& X=(1-z) \hat{Z}_{p} \tilde{G}+Y+\sum_{g \in G} X_{g}, \text { and } \\
& X^{\prime}=\left\langle(1-z)\left(g-g_{r}\right): g \in \tilde{G}\right\rangle+Y+\sum_{g \in G} X_{g} .
\end{aligned}
$$

Note that these are independent of the choice of generator $z \in \operatorname{Ker}(\beta)$.

The idea now is to show that $\beta_{z}^{\#}$ factors through $X / X^{\prime}$ (or, more precisely, $\left.X \cap \operatorname{Im}(\Gamma) / X^{\prime} \cap \operatorname{Im}(\Gamma)\right)$; and then to analyze this group more closely.

Proposition 4.4. For any $u \in K_{1}(\mathrm{ZG})$ and any $\tilde{u} \in \beta_{*}^{-1}\left(i_{*}(u)\right)$ :

(i) $\Gamma(\tilde{u}) \in X$,

(ii) $\Gamma(\tilde{u}) \in X^{\prime}$ if $\beta_{*}(\tilde{u})=i_{*}(u)=0$, and

(iii) $\beta_{z}^{\#}(u)=\partial \circ E_{z}(\tilde{u})$ for any $z$ generating $\operatorname{Ker}(\beta)$.

Proof. By Theorem 4.1 (iii),

$$
\begin{gathered}
\Gamma_{G}\left(i_{*}(u)\right) \in\left\langle g+g^{-1}-g^{m}-g^{-m}:(m,|g|)=1\right\rangle \\
+\left\langle g-g^{m}:(m,|g|)=1, g \operatorname{conj} g^{-1}\right\rangle .
\end{gathered}
$$

If $g$ is conjugate to $g^{-1}$ in $G$, and $(m,|g|)=1$, then in $H_{0}\left(G ; \hat{Z}_{p} G\right)$,

$$
\begin{array}{ll}
g-g^{m}=\frac{1}{2}\left(g+g^{-1}-g^{m}-g^{-m}\right) & \text { if } p \text { is odd } \\
g-g^{m} \in \beta(Y) & \text { if } p=2 \text { and } g \in G_{r} \\
g-g^{m}=g-g^{m}+\left(\frac{m-1}{2}\right)\left(g-g^{-1}\right) \in \beta\left(X_{g}\right) & \text { if } p=2 \text { and } g \notin G_{r} .
\end{array}
$$

Thus, referring to the above definitions,

$$
\Gamma_{G}\left(i_{*}(u)\right) \in \beta(Y)+\sum_{g \in G} \beta\left(X_{g}\right) .
$$

So for any $\tilde{u} \in \beta_{*}^{-1}\left(i_{*}(u)\right)$,

$$
\Gamma_{\tilde{G}}(\tilde{u}) \in \operatorname{Ker}\left[H_{0}\left(\tilde{G} ; Z_{p} \tilde{G}\right) \stackrel{\beta}{\longrightarrow} H_{0}\left(G ; \hat{Z}_{p} G\right)\right]+Y+\sum_{g \in G} X_{g}=X .
$$


This proves (i). Point (ii) follows from Theorem 4.1 (ii) -

$$
\Gamma\left(\operatorname{Ker}\left(\beta_{*}\right)\right)=\Gamma\left(1+(1-z) \hat{Z}_{p} \widetilde{G}\right) \subseteq\left\langle(1-z)\left(g-g_{r}\right)\right\rangle+Y \subseteq X^{\prime}
$$

if $z$ generates $\operatorname{Ker}(\beta)$ - and (iii) follows from Proposition 2.6.

It remains to understand the relationship between $\partial \circ E_{z}(\tilde{u})$ and $\Gamma(\tilde{u})$, for $\tilde{u} \in K_{1}\left(\hat{Z}_{p} \tilde{G}\right)$. The first step is to show,

Proposition 4.5. For any z generating $\operatorname{Ker}(\beta)$, and any $\tilde{u} \in K_{1}\left(\hat{Z}_{p} \widetilde{G}\right)$ such that $\Gamma(\tilde{u}) \in Y, E_{z}(\tilde{u})=0$.

Proof. SteP 1. Note first that $(1-1 / p \Phi)$ is an automorphism of $H_{0}\left(\widetilde{G} ; \hat{Q}_{p} \widetilde{G}\right)$ : this can be checked directly by computing the determinant, or follows since $\Gamma=(1-1 / p \Phi) \cdot \log$ has finite cokernel. Since $\Phi(Y) \subseteq Y$ by definition, it follows that

$$
\log (\tilde{u})=(1-1 / p \Phi)^{-1}(\Gamma(\tilde{u})) \in(1-1 / p \Phi)^{-1}(Y) \subseteq \hat{\mathrm{Q}}_{p} \cdot Y .
$$

So we can write

$$
\log (\tilde{u})=\sum_{i=1}^{k} \lambda_{i}\left(g_{i}-g_{i}^{\prime}\right) \in H_{0}\left(\tilde{G} ; \hat{\mathrm{Q}}_{p} \tilde{G}\right),
$$

where for all $i, \lambda_{i} \in \hat{Q}_{p}$ and $g_{i}, g_{i}^{\prime}$ are $p^{\prime}$-conjugate.

STEP 2. We must show that $\operatorname{pr}_{B^{*}}\left(E_{z}(\tilde{u})\right)=1$ for any simple summand $B$ of $Q \tilde{G}$; where $\operatorname{pr}_{B}: Q \widetilde{G} \rightarrow B$ is the projection. This holds by definition if $\operatorname{pr}_{B}(z)=1$. So it remains to show, when $\operatorname{pr}_{B}(z)=\zeta \neq 1$, that

$$
\operatorname{pr}_{B^{*}}\left(E_{z}(\tilde{u})\right)=\left\{1-\zeta, \operatorname{pr}_{B}(\tilde{u})\right\}=1 \text { in } C_{p}(B) .
$$

Set $K=Z(B)$, let $F \subseteq K$ be the subfield generated by $\left(\mu_{K}\right) p$ (the group of $p$ power roots of unity), and note that $\zeta \in\left(\mu_{K}\right)_{p}=\left(\mu_{F}\right)_{p}$. Set $\hat{K}_{p}=\hat{Q}_{p} \otimes_{Q} K$ and $\hat{F}_{p}=\hat{Q}_{p} \otimes_{Q} F$. The triangle

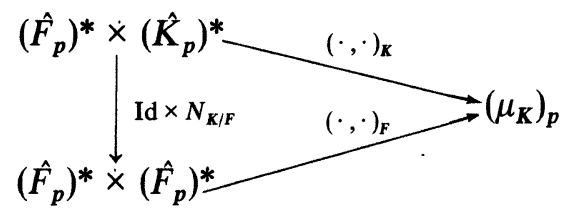

commutes by Theorem 2.4 . So using Theorem $2.2,(2)$ holds if and only if

$$
\left(1-\zeta, N_{K / F} \operatorname{nr}_{B / K}\left(\operatorname{pr}_{B}(\tilde{u})\right)_{F}=1,\right.
$$

where $N_{\boldsymbol{K} / \boldsymbol{F}}$ and $\mathrm{nr}_{\boldsymbol{B} / \mathbf{K}}$ are the ordinary and reduced norm maps.

By Proposition 2.8, and (1), if $r=[B: K]^{1 / 2}$, then 


$$
\log \left(N_{K / F} \operatorname{nr}_{B / K}\left(\operatorname{pr}_{B}(\tilde{u})\right)\right)=1 / r \sum_{i=1}^{k} \lambda_{i}\left(\chi_{B}\left(g_{i}\right)-\chi_{B}\left(g_{i}^{\prime}\right)\right) \in \hat{F}_{p},
$$

where $\chi_{B}$ denotes the character of $B$ regarded as an $F[\widetilde{G}]$-module. In particular, $\chi_{B}$ is constant on $F$-conjugacy classes in $\widetilde{G}$ [10, Corollary 21.3 , where $g, g^{\prime} \in \widetilde{G}$ are called $F$-conjugate if, upon setting $n=|g|, g^{\prime}$ is conjugate to $g^{m}$ for some $m \in \operatorname{Gal}\left(F \zeta_{n} / F\right)\left(\subseteq(Z / n)^{*}\right)$. In particular, $p^{\prime}$-conjugate elements are $F$-conjugate $\left(F \cong Q \zeta_{p} m\right.$ for some $m$ ), and so by (4)

$$
\log \left(N_{K / F} \operatorname{nr}_{B / K}\left(\operatorname{pr}_{B}(\tilde{u})\right)\right)=0 .
$$

Equation (3), and hence (2), now follow using Theorem 2.3 (iii).

We now want to show that $\partial E_{z}(u)=0$ if $\Gamma(u) \in X^{\prime}$, and that $\partial E_{z}(u)$ is independent of $z$ if $\Gamma(u) \in X$. One lemma is still needed.

Lemma 4.6. For any generator $z$ of $\operatorname{Ker}(\beta)$, any $g \in \widetilde{G}$, and any $x \in X_{g}$, there exists $u \in K_{1}\left(\hat{Z}_{p} \widetilde{G}\right)$ such that $\Gamma(u) \in x+Y$ and $\partial E_{z}(u)=0$.

Proof. Fix $g \in \sigma$, and set $H=\langle z, g\rangle$. Set $a=g_{p}$ and $h=g_{r}$; that is $g=a h=h a, a \in \tilde{G}_{p}$, and $h \in \widetilde{G}_{r}$. Set $n=|h|, C_{n}=\langle h\rangle$, and $\pi=\langle z, a\rangle$. Then $H=C_{n} \times \pi$, where $p \nmid n$ and $\pi$ is an abelian $p$-group of rank $\leqq 2$.

Consider the following commutative diagram:

(1)

$$
\begin{aligned}
& K_{1}\left(\hat{Z}_{p} H\right) \stackrel{f_{1}=\prod f_{1}^{d}}{\longrightarrow} \prod_{d \mid n} K_{1}\left(\hat{Z}_{p} \zeta_{d}[\pi]\right) \stackrel{t_{1}=\prod t_{1}^{d}}{\longrightarrow} \prod_{d \mid n} K_{1}\left(\hat{Z}_{p} \pi\right) \\
& \partial_{H} E_{z}^{H} \\
& \mathrm{Cl}_{1}(\mathrm{ZH}) \stackrel{f_{2}}{\cong} \prod_{d \mid n} \mathrm{Cl}_{1}\left(\mathrm{Z} \zeta_{d}[\pi]\right) \stackrel{t_{2}}{\stackrel{t_{\pi} E_{z}^{n}}{\cong}} \prod_{d \mid n} \mathrm{Cl}_{1}(\mathrm{Z} \pi) .
\end{aligned}
$$

Here, $\partial_{H} E_{z}^{H}$ and $\partial_{\pi} E_{z}^{\pi}$ are defined analogously to $\partial E_{z}$ in diagram (4.3). The $f_{i}$ are induced by some isomorphism $\mathrm{Q}\left[C_{n}\right] \cong \prod_{d \mid n} \mathrm{Q} \zeta_{d}$ (recall that we define $\left.\hat{Z}_{p} \zeta_{n}=\hat{Z}_{p} \otimes Z \zeta_{n}\right) ; f_{2}$ is an isomorphism since it is induced by an inclusion of orders of index prime to $p$ (see [20, Proposition 1.2]). The $t_{i}$ are both the products of the transfer homomorphisms, and $t_{2}$ is an isomorphism by Proposition 2.11.

The proof now splits into two separate cases.

CASE 1. If $p>2$, or if $\beta(g)$ is not $p^{\prime}$-conjugate to $\beta\left(g^{-1}\right)$ in $G$, then it suffices to prove (i), when $x=g+g^{-1}-g^{m}-g^{-m}$ for any $(m,|g|)=1$. Fix such an $m$, and set

$$
x^{\prime}=\left(a+a^{-1}-a^{m}-a^{-m}\right) h \in X+Y\left(a=g_{p}, h=g_{r}\right) .
$$

By Theorem 4.1 there exists $u \in\left(Z_{p} H\right)^{*}$ such that $\Gamma_{H}(u)=x^{\prime}$. For any $d \mid n$, 


$$
\Gamma_{\pi}\left(t_{1}^{d} f_{1}^{d}(u)\right)=\lambda\left(a+a^{-1}-a^{m}-a^{-m}\right)
$$

for some $\lambda \in \hat{Z}_{p}$. Hence, $\partial_{\pi} E_{z}^{\pi} t_{1}^{d} f_{1}^{d}(u)=0$ by Lemma 4.2 ; and so $\partial_{H} E_{z}^{H}(u)$ $=0$ in diagram (1). By naturality, $\partial E([u])=0$ and $\Gamma([u])=x^{\prime}$, where $[u] \in K_{1}\left(\hat{Z}_{p} \widetilde{G}\right)$ denotes the class of $u$.

CASE 2. Assume now that $p=2$, and that $\beta(g)$ is $p^{\prime}$-conjugate to $\beta\left(g^{-1}\right)$ in $G$. Fix $n \in N(H)$ such that $n a n^{-1}=z^{i} a^{-1}\left(a=g_{p}\right)$, and let $\eta \in \operatorname{Aut}(H)$ denote the automorphism conjugation by $n$.

Fix any $x=\sum_{i=1}^{k} \lambda_{i} g^{i} \in X_{g}$ : that is, $\lambda_{i}=0$ if $(i,|g|) \neq 1, \sum \lambda_{i}=0$, and $\sum i \lambda_{i}$ $\equiv 0(\bmod |g|)$. Set

$$
x^{\prime}=\sum_{i=1}^{k} \lambda_{i} a^{i} h \in x+Y \quad\left(a=g_{p}, h=g_{r}\right) .
$$

Then $x^{\prime} \in \operatorname{Im}\left(\Gamma_{H}\right)$ by Theorem 4.1; fix $u \in\left(\hat{Z}_{p} H\right)^{*}$ such that $\Gamma_{H}(u)=x^{\prime}$. By Lemma 4.2 (ii), for any $d \mid n$,

$$
\partial_{\pi} E_{z}^{\pi} t_{1}^{d} f_{1}^{d}(u) \in\left(1-\eta_{*}\right) \mathrm{Cl}_{1}(\mathrm{Z} \pi)
$$

in the notation of (1) above. It follows that $\partial_{H} E_{z}^{H}(u) \in\left(1-\eta_{*}\right) \mathrm{Cl}_{1}(\mathrm{ZH})$. But $\eta$ extends to an inner automorphism of $\tilde{G}$, and hence $\partial E_{z}([u])=0$ in $\mathrm{Cl}_{1}(\mathrm{Z} \widetilde{G})$.

We are now ready to prove:

Proposition 4.7. For any $u \in K_{1}\left(\hat{Z}_{p} \tilde{G}\right)$ and any $z, z^{\prime}$ generating $\sigma=\operatorname{Ker}(\beta)$ :

(i) $\partial E_{z}(u)=0$ if $\Gamma(u) \in X^{\prime}$, and

(ii) $\partial E_{z}(u)=\partial E_{z^{\prime}}(u)$ if $\Gamma(u) \in X$.

Proof. (i) Assume $\Gamma(u) \in X^{\prime}$. Since $\left\langle(1-z)\left(g-g_{r}\right)\right\rangle \subseteq \Gamma\left(1+(1-z) \hat{Z}_{p} \tilde{G}\right)$ (Theorem 4.1 (ii); we can write

$$
\Gamma(u) \equiv \Gamma(1+(1-z) \xi)+\sum_{g \in G} x_{g}(\bmod Y),
$$

where $x_{g} \in X_{g}$ for all $g$ (and $\xi \in \hat{Z}_{p} \tilde{G}$ ). By Lemma 4.6, there are elements $u_{g} \in K_{1}\left(Z_{p} \widetilde{G}\right)$ such that $\Gamma\left(u_{g}\right) \in x_{g}+Y$ and $\partial E_{z}\left(u_{g}\right)=0$.

If we now set

$$
u^{\prime}=u \cdot\left(\prod_{g \in G} u_{g}\right)^{-1} \cdot(1+(1-z) \xi)^{-1},
$$

then $\Gamma\left(u^{\prime}\right) \in Y$. By Proposition 4.5, $\partial E_{z}\left(u^{\prime}\right)=0$, and so 


$$
\partial E_{z}(u)=\partial E_{z}(1+(1-z) \xi)+\sum_{g \in \widetilde{G}} \partial E_{z}\left(u_{g}\right)=\partial E_{z}(1+(1-z) \xi) .
$$

So by Proposition 4.4 (iii) (applied with $u=1$ ),

$$
\partial E_{z}(u)=\partial E_{z}(1+(1-z) \xi)=\beta_{z}^{\#}(1)=0 .
$$

(ii) Now assume that $\Gamma(u) \in X$, and that $z, z^{\prime}$ generate $\operatorname{Ker}(\beta)$. Fix $m$ such that $(m,|G|)=1$ and $z^{\prime}=z^{m}$. Define

$$
\Phi_{m}: H_{0}\left(\tilde{G} ; \hat{Z}_{p} \widetilde{G}\right) \rightarrow H_{0}\left(\tilde{G} ; \hat{Z}_{p} \widetilde{G}\right), \eta_{m} \in \operatorname{Gal}\left(Q\left(\zeta_{|G|}\right) / Q\right)
$$

by setting

$$
\Phi_{m}\left(\sum \lambda_{i} g_{i}\right)=\sum \lambda_{i} g_{i}^{m} \text {, and } \eta_{m}(\zeta)=\zeta^{m} \text { if } \zeta=\zeta_{|G|}
$$

From Theorem 4.1 (i), we see that $\operatorname{Im}(\Gamma)=\operatorname{Ker}(\bar{\omega}, \bar{\theta})$ is stable under $\Phi_{m}$. In particular, we can choose $v \in K_{1}\left(\hat{Z}_{p} \widetilde{G}\right)$ such that $\Gamma(v)=\Phi_{m}(\Gamma(u))$. Also, $\Phi_{m}$ commutes with $(1-1 / p \Phi)$, and so $\log (v)=\Phi_{m}(\log (u))$ in $H_{0}\left(\widetilde{G} ; \hat{Q}_{p} \tilde{G}\right)$. Fix $\lambda_{i} \in \hat{Q}_{p}, g_{i} \in \tilde{G}$ such that

$$
\log (u)=\sum_{i=1}^{k} \lambda_{i} g_{i}, \log (v)=\sum_{i=1}^{k} \lambda_{i} g_{i}^{m}
$$

Let $B$ be any simple summand of $Q \tilde{G}$ such that $\operatorname{pr}_{B}(z) \neq 1$, and set $K=Z(B)$. By Proposition 2.8,

$$
\begin{aligned}
\log \left(\operatorname{nr}_{B / K}\left(\operatorname{pr}_{B}(v)\right)\right) & =[B: K]^{-1 / 2} \cdot \sum_{i=1}^{k} \lambda_{i} \chi_{B}\left(g_{i}^{m}\right) \\
& =[B: K]^{-1 / 2} \cdot \sum_{i=1}^{k} \lambda_{i} \cdot \eta_{m}\left(\chi_{B}\left(g_{i}\right)\right) \\
& =\eta_{m}\left(\log \left(\operatorname{nr}_{B / K} \operatorname{pr}_{B}(u)\right)\right) \in \hat{K}_{p} .
\end{aligned}
$$

Symbols with 1- $\operatorname{pr}_{B}(z)$ depend only on the logarithm (Theorem 2.3), and so (by naturality of symbols in $K$ ):

$$
\left(1-\operatorname{pr}_{B}\left(z^{m}\right), \operatorname{nr}_{B / K} \operatorname{pr}_{B}(v)\right)_{K}=\left(1-\operatorname{pr}_{B}(z), \mathrm{nr}_{B / K} \operatorname{pr}_{B}(u)\right)_{K}^{m}
$$

By Theorem 2.2, it follows that $E_{z^{\prime}}(v)=m \cdot E_{z}(u)\left(z^{\prime}=z^{m}\right)$, and so

$$
\partial E_{z^{\prime}}(u)-\partial E_{z}(u)=1 / m \partial E_{z^{\prime}}\left(u^{m} v^{-1}\right) .
$$

By construction,

$$
\Gamma\left(u^{m} v^{-1}\right)=m \Gamma(u)-\Phi_{m}(\Gamma(u)) \in\left(m-\Phi_{m}\right)(X) .
$$

Furthermore, $\left(m-\Phi_{m}\right) \cdot(1-z) \hat{Z}_{p} \tilde{G}$ is generated by elements 


$$
\begin{aligned}
m(1-z) g-\left(1-z^{m}\right) g^{m} & =(1-z)\left(m-\left(1+z+\ldots+z^{m-1}\right)\right) g \\
& +\left(1-z^{m}\right)\left(g-g^{m}\right) \\
& \subseteq\left\langle(1-z)\left(g-g_{r}\right)\right\rangle+Y \subseteq X^{\prime}
\end{aligned}
$$

for $g \in \tilde{G}$. Also, $\Phi_{m}\left(Y+\sum X_{g}\right)=Y+\sum X_{g}$, and so

$$
\Gamma\left(u^{m} v^{-1}\right) \in\left(m-\Phi_{m}\right)(X) \subseteq Y+\sum X_{g}+\left(m-\Phi_{m}\right)(1-z) \hat{Z}_{p} \widetilde{G} \subseteq X^{\prime} .
$$

Hence $\partial E_{z^{\prime}}\left(u^{m} v^{-1}\right)=0$ by part (i), and so $\partial E_{z^{\prime}}(u)=\partial E_{z}(u)$ by (2).

Propositions 4.4 and 4.7 show that $\beta_{z}^{\#}$ is independent of $z$, and factors through $X \cap \operatorname{Im}(\Gamma) / X^{\prime} \cap \operatorname{Im}(\Gamma)$. But before stating this explicitly, a better description of this obstruction group will be useful.

Let $\widetilde{G}_{r}^{*}$ denote the set of Q-conjugacy classes of $p$-regular elements $h \in \widetilde{G}_{r}$ ( $h, h^{\prime}$ are Q-conjugate if $\langle h\rangle$ and $\left\langle h^{\prime}\right\rangle$ are conjugate). The Q-conjugacy class of any $h \in \widetilde{G}_{r}$ will be denoted $(h) \in \widetilde{G}_{r}^{*}$. As usual, $N(h)=N_{\tilde{G}}(h)$ and $C(h)$ $=C_{\widetilde{G}}(h)$ denote the normalizer and centralizer of $\langle h\rangle$.

Proposition 4.8. Define

$$
\begin{aligned}
W_{\beta} & =\prod_{(h) \in \widetilde{G}_{r}^{*}}(\operatorname{Ker}(\beta) \cap[N(h), C(h)]) \cdot(h), \text { and } \\
W_{\beta}^{\prime} & =\left\langle(\operatorname{Ker}(\beta) \cap[N(H), H]) \cdot\left(g_{r}\right): g \in \widetilde{G}, H=\langle\operatorname{Ker}(\beta), g\rangle\right\rangle \subseteq W_{\beta} .
\end{aligned}
$$

Then

(i) for any $h \in \tilde{G}_{r}$,

$\operatorname{Ker}(\beta) \cap[N(h), C(h)]=\left\{w \in \operatorname{Ker}(\beta):(1-w) h \in \operatorname{Im}(\Gamma)+X^{\prime}\right\}$.

(ii) The function

$$
T_{\beta}: W_{\beta} / W_{\beta}^{\prime} \stackrel{\cong}{\longrightarrow} \frac{X \cap \operatorname{Im}(\Gamma)}{X^{\prime} \cap \operatorname{Im}(\Gamma)},
$$

defined by setting

$$
T_{\beta}(w \cdot(h))=(1-w) h+x^{\prime}
$$

for any $h \in \widetilde{G}_{r}, w \in \operatorname{Ker}(\beta) \cap[N(h), C(h)]$, and $x^{\prime} \in X^{\prime}$ such that $(1-w) h$ $+x^{\prime} \in \operatorname{Im}(\Gamma)$, is an isomorphism.

(iii)

$$
W_{\beta}^{\prime} \stackrel{2}{\underline{2}}\langle[n, g] \cdot(h): n \in N(h), g \in C(h),[n, g] \in \operatorname{Ker}(\beta)\rangle,
$$

with equality if $p$ is odd. 
Proof. For each $(h) \in \widetilde{G}_{r}^{*}$, define

$$
U_{h}=\left\{\sum_{i=1}^{k} \lambda_{i} g_{i}: \lambda_{i} \in \hat{Z}_{p}, g_{i} \in \tilde{G},\left(\left(g_{i}\right)_{r}\right)=(h) \text { all } i\right\} \subseteq H_{0}\left(\tilde{G} ; \hat{Z}_{p} \tilde{G}\right),
$$

and set

$$
X_{h}=X \cap U_{h}, X_{h}^{\prime}=X^{\prime} \cap U_{h}, \operatorname{Im}(\Gamma)_{h}=\operatorname{Im}(\Gamma) \cap U_{h} \text {, etc. }
$$

Then from the definitions (or from the description of $\operatorname{Im}(\Gamma)$ in Theorem 4.1) we get

$$
H_{0}\left(\tilde{G} ; \hat{Z}_{p} \tilde{G}\right)=\bigoplus_{(h) \in \tilde{G}_{r}^{*}} U_{h}, X=\bigoplus_{(h)} X_{h}, X^{\prime}=\bigoplus_{(h)} X_{h}^{\prime}, Y=\bigoplus_{(h)} Y_{h},
$$

$$
\operatorname{Im}(\Gamma)=\bigoplus_{(h)} \operatorname{Im}(\Gamma)_{h}, W_{\beta}=\prod_{(h)} W_{\beta, h} \cdot(h), W_{\beta}^{\prime}=\prod_{(h)} W_{\beta, h}^{\prime} \cdot(h) .
$$

Throughout the proof, $z \in \operatorname{Ker}(\beta)$ denotes a fixed generator.

STEP 1. Recall the description of $\operatorname{Im}(\Gamma)$ in Theorem 4.1:

$$
\operatorname{Im}(\Gamma)=\operatorname{Ker}\left[(\bar{\omega}, \bar{\theta}): H_{0}\left(\tilde{G} ; \hat{Z}_{p} \tilde{G}\right) \rightarrow H_{1}\left(\tilde{G} ; \hat{Z}_{p}\left(\tilde{G}_{r}\right)\right)_{\Phi} \oplus H_{0}\left(\tilde{G} ; R\left(\tilde{G}_{r}\right)\right)_{\Phi}\right],
$$

where $R=Z / 2(p=2)$ or $R=0(p>2)$. In particular, for any $h \in \widetilde{G}_{r}$,

$$
\begin{aligned}
\{W & \left.\in \operatorname{Ker}(\beta):(1-w) h \in \operatorname{Im}(\Gamma)+X^{\prime}\right\} \\
& =\left[\left\{w \in \operatorname{Ker}(\beta):(\bar{\omega}, \bar{\theta})((1-w) h)=\left(w^{-1} \otimes h, 0\right) \in(\bar{\omega}, \bar{\theta})\left(X^{\prime}\right)\right\} .\right.
\end{aligned}
$$

Furthermore, $(\bar{\omega}, \bar{\theta})\left(X_{g}\right) \subseteq(\bar{\omega}, \bar{\theta})(Y)$ for any $g\left(X_{g} \subseteq Y+\operatorname{Im}(\Gamma)\right.$ by Lemma 4.6), and $(\bar{\omega}, \bar{\theta})\left((1-z)\left(g-g_{r}\right)\right)=0$ for any $g \in \widetilde{G}$. Hence

$$
\begin{aligned}
(\bar{\omega}, \bar{\theta})\left(X^{\prime}\right)= & (\bar{\omega}, \bar{\theta})(Y) \\
= & \left\langle\left(a \otimes\left(h-h^{\prime}\right), 0\right),\left(0, h-h^{\prime}\right):\right. \\
& \left.\langle h\rangle=\left\langle h^{\prime}\right\rangle \subseteq G_{r}, a \in G_{p} \cap C(h)\right\rangle,
\end{aligned}
$$

and so

$$
\begin{aligned}
& {\left[H_{1}\left(\tilde{G} ; \hat{Z}_{p}\left(\tilde{G}_{r}\right)\right)_{\Phi} \oplus H_{0}\left(\tilde{G} ; R\left(\tilde{G}_{r}\right)\right)_{\Phi}\right] /(\bar{\omega}, \bar{\theta})\left(X^{\prime}\right)} \\
& \cong H_{0}\left(\tilde{G} ; \bigoplus_{\langle h\rangle}(C(h))^{\mathrm{ab}} \otimes \hat{Z}_{p}\right) \oplus H_{0}\left(G ; \bigoplus_{\langle h\rangle} R\right) \\
& \left.\cong\left(\bigoplus_{(h)}(C(h) /[N(h), C(h)]) \otimes \hat{Z}_{p}\right)\right) \oplus\left(\bigoplus_{(h)} R\right) \text {. }
\end{aligned}
$$

Together with (1) and (2), this proves that for any $(h) \in G_{r}^{*}$, (3) $\left\{w \in \operatorname{Ker}(\beta):(1-w) h \in \operatorname{Im}(\Gamma)_{h}+X_{h}^{\prime}\right\}=\operatorname{Ker}(\beta) \cap[N(h), C(h)]=W_{\beta, h}$. 
STEP 2. By (3), there is a function

$$
\widetilde{T}_{\beta}: W_{\beta}=\bigoplus_{(h)} W_{\beta, h} \cdot(h) \rightarrow \frac{X \cap \operatorname{Im}(\Gamma)}{X^{\prime} \cup \operatorname{Im}(\Gamma)}
$$

defined by setting, for any $h \in \widetilde{G}_{r}$ and any $w \in W_{\beta, h}$,

$$
\widetilde{T}_{\beta}(w \cdot(h))=(1-w) h+x^{\prime} \quad\left(x^{\prime} \in X_{h}^{\prime},(1-w) h+x^{\prime} \in \operatorname{Im}(\Gamma)_{h}\right) .
$$

Then $\widetilde{T}_{\beta}$ is a homomorphism, since

$$
\begin{gathered}
\left.(1-v) h+(1-w) h-(1-v w) h=(1-v)(1-w) h \in\langle 1-z)\left(g-g_{r}\right)\right\rangle \\
\subseteq X^{\prime} \cap \operatorname{Im}(\Gamma),
\end{gathered}
$$

for any $v, w \in W_{\beta, h}$. Furthermore, for any $h \in \widetilde{G}_{r}$,

$$
\begin{aligned}
X_{h} \cap \operatorname{Im}(\Gamma)_{h} & =\left[X_{h}^{\prime}+\{(1-w) h: w \in \operatorname{Ker}(\beta)\}\right] \cap \operatorname{Im}(\Gamma)_{h} \\
& =\left[X_{h}^{\prime}+\left\{(1-w) h: w \in W_{\beta, h}\right\}\right] \cap \operatorname{Im}(\Gamma),
\end{aligned}
$$

by (3), and this proves that $\widetilde{T}_{\beta}$ is surjective.

STEP 3. Fix $w \in \operatorname{Ker}(\beta)$ and $h \in \widetilde{G}_{r}$ such that $w \cdot(h) \in W_{\beta}^{\prime}$. Then there exists $g \in \tilde{G}$ such that $g_{r}=h$, and such that $w \in[N(H), H]$, when $H=\langle z, g\rangle$. We must show that $(1-w) h \in X^{\prime}$.

Set $a=g_{p}$. For any $n_{1}, n_{2} \in N(H)$ and any $g^{i} z^{i} \in H$,

$\left[n_{1}, g^{i} z^{j}\right]=\left[n_{1}, a\right]^{i} ;\left[n_{1} n_{2}, g^{i} z^{j}\right]=\left[n_{1}, n_{2} a^{i} n_{2}^{-1}\right]\left[n_{2}, a^{i}\right] \in\left\langle\left[n_{1}, a\right],\left[n_{2}, a\right]\right\rangle$

( $H$ is abelian). So if we set

$$
N_{0}=\{n \in N(H):[n, a] \in\langle z\rangle\}=\operatorname{Ker}\left[N(H) \stackrel{\text { conj }}{\longrightarrow} \operatorname{Aut}\left(H_{p} / z\right)\right],
$$

and if $N(H)=\left\langle n_{1}, n_{2}, N_{0}\right\rangle$, then

$$
\begin{aligned}
\operatorname{Ker}(\beta) \cap[N(H), H] & =\left\{\left[n, a^{i}\right]: n \in N_{0}, i \in Z\right\} \\
& \cap\left(\operatorname{Ker}(\beta) \cap\left\langle\left[n_{1}, a\right],\left[n_{2}, a\right]\right\rangle\right) .
\end{aligned}
$$

If $N(H) / N_{0}$ is cyclic, then by (4), $w=\left[n, a^{i}\right]$ for some $n \in N(H)$ and some $i \in Z$. Since $\left\langle n^{-1} h n\right\rangle=\langle h\rangle\left(H_{r}=\langle h\rangle\right)$, it follows that

$$
\begin{aligned}
(1-w) h & =(1-w)\left(1-a^{i}\right) h+\left(a^{i} h-w a^{i} h\right) \\
& =(1-w)\left(1-a^{i}\right) h+a^{i}\left(h-n^{-1} h n\right) \in X^{\prime} .
\end{aligned}
$$

If $N(H) / N_{o}\left(\subseteq \operatorname{Aut}\left(H_{p} / z\right)\right)$ is not cyclic, then $p=2$, and $a=g_{p}$ is conjugate in $N(H)$ to $a^{-1} z^{i}$ for some $i$. So in this case,

$$
X_{g}=\left\{\sum_{i=1}^{k} \lambda_{i} g^{i}: \lambda_{i}=0 \text { if }(|g|, i) \neq 1, \sum \lambda_{i}=0, \sum i \lambda_{i} \equiv 0\left(\bmod \left|g_{p}\right|\right)\right\} .
$$


By (4), either $w=\left[n, a^{i}\right]$ for some $n, i$ (and $(1-w) h \in X^{\prime}$ as in (5)); or $w=\left[n_{1}, a^{i}\right]\left[n_{2}, a^{j}\right]$ for some $n_{1}, n_{2} \in N(H)$. In the latter case, if we set $b_{1}=\left[n_{1}, a^{i}\right]$ and $b_{2}=\left[n_{2}, a^{j}\right]\left(b_{1}, b_{2} \in H_{p}\right)$, then

$$
\begin{aligned}
(1-w) h & =\left(1-b_{1}\right) h+\left(1-b_{2}\right) h-\left(1-b_{1}\right)\left(1-b_{2}\right) h \\
& =\left(1-b_{1}\right)\left(1-a^{i}\right) h+\left(1-b_{2}\right)\left(1-a^{j}\right) h+a^{i}\left(h-n_{1}^{-1} h n_{1}\right) \\
& +a^{j}\left(h-n_{2}^{-1} h n_{2}\right)-\left(1-b_{1}\right)\left(1-b_{2}\right) h \\
& \in Y+\left\langle(1-b)\left(1-b^{\prime}\right) h: b, b^{\prime} \in H_{p}\right\rangle \\
& \subseteq Y+X_{g}+\left\langle(1-z)\left(a^{i} z^{j}-1\right) h\right\rangle \subseteq X^{\prime} .
\end{aligned}
$$

Thus, in both cases, $(1-w) h \in X^{\prime}$, and so $\tilde{T}_{\beta}$ factors through an epimorphism $T_{\beta}$ defined on $W_{\beta} / W_{\beta}^{\prime}$.

STEP 4. Now set $\mathscr{H}=\{H \subseteq \widetilde{G}: H=\langle z, g\rangle$, some $g \in \tilde{G}\}$, and note that all elements of $\mathscr{H}$ are abelian. Define

$$
R=\left(\prod_{H \in \mathscr{H}} H_{p}\right) /\left\langle(z, H)-\left(z, H^{\prime}\right): H, H^{\prime} \in \mathscr{H}, H_{p}=H_{p}^{\prime}\right\rangle .
$$

Then $\tilde{G}$ acts on $R$ via $g(h, H)=\left(g h g^{-1}, g H g^{-1}\right)$, and we set $\bar{R}=H_{0}(G ; R)$. In particular, if $H_{1}, \ldots, H_{t}$ are conjugacy class representatives for $\mathscr{H}$, then

$$
\bar{R} \cong\left(\prod_{i=1}^{t}\left(H_{i} /\left[N\left(H_{i}\right), H_{i}\right]\right)\right) /\left\langle\left(z, H_{i}\right)-\left(z, H_{j}\right): H_{i r} \operatorname{conj} H_{j r}\right\rangle .
$$

Define $f: H_{0}\left(\tilde{G} ; \hat{Z}_{p} \tilde{G}\right) \rightarrow \bar{R}$ by setting $f(g)=\left(g_{p},\langle z, g\rangle\right) \in \bar{R}$ for $g \in \widetilde{G}$. From the definition, $f\left(X^{\prime}\right)=0$ : note, for example, that

$$
f\left((1-z)\left(g-g_{r}\right)\right)=\left(g_{p}, H\right)-\left(z g_{p}, H\right)+\left(z, H_{0}\right)=-(z, H)+\left(z, H_{0}\right)=0
$$

if $g \in \tilde{G}, H=\langle g, z\rangle$, and $H_{0}=\left\langle g_{r}, z\right\rangle$. So if $w \in \operatorname{Ker}(\beta)$ and $h \in \widetilde{G}_{r}$ are such that $(1-w) h \in X^{\prime}$, then $(w,\langle z, h\rangle)=-f((1-w) h)=0$, and by $(6)$

$$
w \in\left\langle\operatorname{Ker}(\beta) \cap[N(H), H]: H \in \mathscr{H}, H_{r}=\langle h\rangle\right\rangle=W_{\beta, h}^{\prime} .
$$

By (1), $\operatorname{Ker}\left(T_{\beta}\right)$ is generated by such $w \cdot(h)$, and so $T_{\beta}$ is injective.

STEP 5. It remains to prove point (iii). That

$$
W_{\beta}^{\prime} \supseteq\langle[n, g] \cdot(h): n \in N(h), g \in C(h),[n, g] \in \operatorname{Ker}(\beta)\rangle
$$

is clear from the definition. If $p$ is odd, then for any $H=\langle z, g\rangle, N(H) / N_{0}$ is cyclic (in the notation of Step 3), and so (7) is an equality by (4).

THEOREM 4.9. Let $\beta: G \rightarrow G$ be any surjection of finite groups such that $\operatorname{Ker}(\beta) \subseteq Z(G)$, and is cyclic of p-power order. Let $\beta_{z}^{\#}: K_{1}(Z G) \rightarrow \mathrm{Cl}_{1}(\mathrm{Z} \widetilde{G})$ (any $z$ generating $\operatorname{Ker}(\beta)$ ) denote the induced transfer homomorphism, and let 


$$
\begin{aligned}
& W_{\beta}=\underset{(h)}{\bigoplus}(\operatorname{Ker}(\beta) \cap[N(h), C(h)]) \cdot(h) \\
& W_{\beta}^{\prime}=\left\langle\operatorname{Ker}(\beta) \cap[N(H), H] \cdot\left(g_{r}\right): g \in \widetilde{G}, H=\langle z, g\rangle\right\rangle \subseteq W_{\beta}
\end{aligned}
$$

be the groups defined in Proposition 4.8. Then

(i) there are well defined homomorphisms

$$
K_{1}(\mathrm{ZG}) \stackrel{\Omega_{\beta}}{\longrightarrow} W_{\beta} / W_{\beta}^{\prime} \stackrel{\Theta_{\beta}}{\longrightarrow} \mathrm{Cl}_{1}(\mathrm{Z} \widetilde{G})
$$

defined by the relations (in the notation of (4.3)):

(a) $\Omega_{\beta}(u)=\sum_{i=1}^{k} w_{i} \cdot\left(h_{i}\right)$ for any $u \in K_{1}(Z G)$, any $\tilde{u} \in \beta_{*}^{-1}\left(i_{*}(u)\right)$, and any $w_{i} \in \operatorname{Ker}(\beta), h_{i} \in \widetilde{G}_{r}$ such that

$$
\Gamma(\tilde{u}) \equiv \sum_{i=1}^{k}\left(1-w_{i}\right) h_{i}\left(\bmod X^{\prime}\right)
$$

(b) $\Theta_{\beta}(w \cdot(h))=E_{z}(\tilde{u})$ for any $h \in \widetilde{G}_{r}$, any $w \in \operatorname{Ker}(\beta) \cap[N(h), C(h)]$, any $z$ generating $\operatorname{Ker}(\beta)$, and any $\tilde{u} \in K_{1}\left(\hat{Z}_{p} \tilde{G}\right)$ such that

$$
\Gamma(\tilde{u}) \equiv(1-w) h\left(\bmod X^{\prime}\right) .
$$

(ii) $\beta_{z}^{\#}=\Theta_{\beta} \circ \Omega_{\beta}$, and is independent of $z$,

(iii)

$$
\Omega_{\beta}\left(S K_{1}(Z G)\right)=\bigoplus_{(h)}(\operatorname{Ker}(\beta) \cap[C(h), C(h)]) \cdot(h)
$$

(iv) $2 \operatorname{Im}\left(\Omega_{\beta}\right) \subseteq \Omega_{\beta}\left(S K_{1}(Z G)\right)$.

Proof. (i) For any $z$, define homomorphisms

$$
K_{1}(\mathrm{ZG}) \stackrel{\hat{\Omega}}{\longrightarrow} \frac{X \cap \operatorname{Im}(\Gamma)}{X^{\prime} \cap \operatorname{Im}(\Gamma)} \stackrel{\Theta}{\longrightarrow} \mathrm{Cl}_{1}(\mathrm{Z} \tilde{G})
$$

by setting: $\hat{\Omega}(u)=\Gamma(\tilde{u})$ for any $u \in K_{1}(Z G)$ and $\tilde{u} \in \beta_{*}^{-1}\left(i_{*}(u)\right)$; and $\hat{\Theta}(x)$ $=E_{z}(\tilde{u})$ for any $x \in X \cap \operatorname{Im}(\Gamma)$ and $\tilde{u} \in \Gamma^{-1}(x)$. Then $\hat{\Omega}$ is well defined by Proposition 4.4 (i. ii), and $\hat{\Theta}$ is well defined and independent of $z$ by Proposition 4.7. Now $\Omega_{\beta}$ and $\Theta_{\beta}$ are defined by composing with the isomorphism of Proposition 4.8.

(ii) By Proposition 4.4 (iii), $\beta_{z}^{\#}=\hat{\Theta} \circ \hat{\Omega}=\Theta_{\beta} \circ \Omega_{\beta}$ for any $z$. Since $\Theta_{\beta}$ and $\Omega_{\beta}$ do not depend on $z$, neither does $\beta_{z}^{\#}$.

(iii) By [19, Theorem 3], $S K_{1}(Z G)_{(p)}$ is generated by induction from $p$-elementary subgroups of $G$. It follows that

$$
\Omega_{\beta}\left(S K_{1}(Z G)\right) \subseteq \bigoplus_{(h)}(\operatorname{Ker}(\beta) \cap[C(h), C(h)]) \cdot(h):
$$


this is the subgroup of $W_{\beta}$ generated by induction from $p$-elementary subgroups of $G$ and $\tilde{G}$.

To prove the opposite inclusion, we use the exact sequence

$$
\begin{aligned}
0 \rightarrow S K_{1}\left(\hat{Z}_{p} \tilde{G}\right) \rightarrow K_{1}\left(\hat{Z}_{p} \widetilde{G}\right) \stackrel{(\Gamma, \omega, \Phi \hat{\theta})}{\longrightarrow} H_{0}\left(\widetilde{G} ; \hat{Z}_{p} \widetilde{G}\right) \oplus H_{1}\left(\widetilde{G} ; \hat{Z}_{p}\left(\widetilde{G}_{r}\right)\right) \\
\oplus H_{0}\left(\tilde{G} ; R\left(\tilde{G}_{r}\right)\right) \stackrel{\left(\begin{array}{lll}
v & \Phi-1 & 0 \\
\theta & 0-1
\end{array}\right)}{\longrightarrow} H_{1}\left(\widetilde{G} ; \hat{Z}_{p}\left(\widetilde{G}_{r}\right)\right) \oplus H_{0}\left(\widetilde{G} ; R\left(\widetilde{G}_{r}\right)\right) \rightarrow 0
\end{aligned}
$$

of [22, Theorem 1.7]. Here, $R=Z / 2(p=2)$ or $R=0(p>2)$, and

$$
(v, \theta)\left(\sum \lambda_{i} g_{i}\right)=\left(\sum g_{i} \otimes \lambda_{i}\left(g_{i}\right)_{r}, \sum \bar{\lambda}_{i}\left(g_{i}\right)_{r}\right)\left(\lambda_{i} \in \hat{Z}_{p}, g_{i} \in \tilde{G}, \bar{\lambda}_{i} \in R\right) .
$$

In particular, for any $h \in \widetilde{G}_{r}$ and any $w \in \operatorname{Ker}(\beta) \cap[C(h), C(h)]$, we can choose $\tilde{u} \in K_{1}\left(\hat{Z}_{p} \tilde{G}\right)$ such that

$$
(\Gamma, \omega, \Phi \hat{\theta})(\tilde{u})=((1-w) h, 0,0) .
$$

Then $\beta_{*}(\tilde{u}) \in S K_{1}\left(\hat{Z}_{p} G\right)$. By Theorem 2.1, there exists $u \in S K_{1}(Z G)$ such that $i_{*}(u)=\beta_{*}(\tilde{u})$, and $\Omega_{\beta}(u)=w \cdot(h)$ by definition.

(iv) That $\left.\exp \left[\operatorname{Im}\left(\Omega_{\beta}\right) / \Omega_{\beta}\left(S K_{1} Z G\right)\right)\right] \leqq 2$ follows by symmetry: $\mathrm{Wh}^{\prime}(G)$ $=\mathrm{Wh}(G) / S K_{1}(\mathrm{Z} G)$ is fixed under the evolution $g \rightarrow g^{-1}$ (see $[30$, Proposition 10.1]); while by construction, $\Omega_{\beta}(\bar{u})=\Omega_{\beta}(u)^{-1}$ for $u \in K_{1}(Z G)$.

Note that $W_{\beta} / W_{\beta}^{\prime}$ is defined purely combinatorially, without reference to $K$-theory. The point of Theorem 4.9 is that it breaks up the problem of describing $\operatorname{Im}\left(\beta^{\#}\right)$ into two independent problems: those of describing $\operatorname{Im}\left(\Omega_{\beta}\right)$ and $\operatorname{Ker}\left(\Theta_{\beta}\right)$.

The main question in studying $\operatorname{Im}\left(\Omega_{\beta}\right)$ is whether it ever can be strictly larger than $\Omega_{\beta}\left(S K_{1}(Z G)\right)$ when $p=2$. Examples of this surely exist but constructing one seems quite hard. Note that by Theorem $4.9, \operatorname{Im}\left(\Omega_{\beta}\right)$ $=\Omega_{\beta}\left(S K_{1}(Z G)\right)$ if $p$ is odd or if $G$ is 2-elementary.

As for $\operatorname{Ker}\left(\Theta_{\beta}\right)$, we will show in a later paper that given any family of central extensions

$$
1 \rightarrow\left\langle z_{n}\right\rangle \rightarrow \tilde{G}_{n} \stackrel{\beta_{n}}{\rightarrow} G \rightarrow 1,
$$

where $\tilde{G}_{n}=\left\langle z_{n}, \widetilde{G}_{n-1}: z_{n-1}=z_{n}^{p},\left[z_{n}, \widetilde{G}_{n-1}\right]=1\right\rangle$; then in the $p$-group case at least, $\Theta_{\beta_{n}}$ is injective for $n$ large enough. Note that in this situation, $W_{\beta_{n}}$ and $W_{\beta_{n}}^{\prime}$ are independent of $n$.

Before listing consequences of Theorem 4.9, the case where $\operatorname{Ker}(\beta)$ is not a $p$-group must be handled. In fact, this can always be directly reduced to the case we have been studying.

THEOREM 4.10. Let $1 \rightarrow\langle z\rangle \rightarrow \widetilde{G} \stackrel{\beta}{\rightarrow} G \rightarrow 1$ be a central extension of finite groups. Write $|z|=p^{r} m$, where $p \nmid m$, and let $C_{m} \varsigma\langle z\rangle=\operatorname{Ker}(\beta)$ be the 
subgroup of order m. Let $\Sigma_{m} \in \mathrm{Z} \widetilde{G}$ be the sum of the elements of $C_{m}$. Then the following triangle commutes,

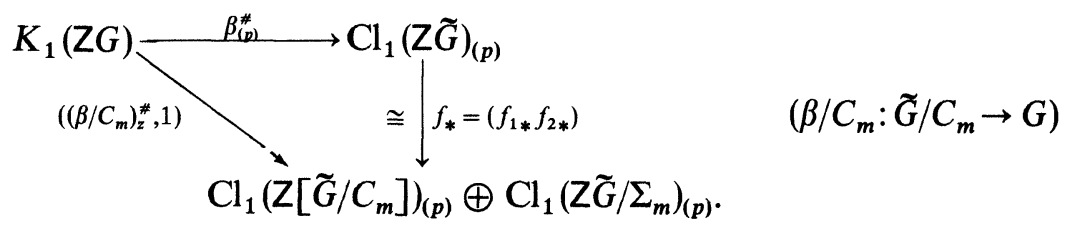

Here, $f_{*}$ is induced by the projections and is an isomorphism.

Proof. Consider the following commutative triangle

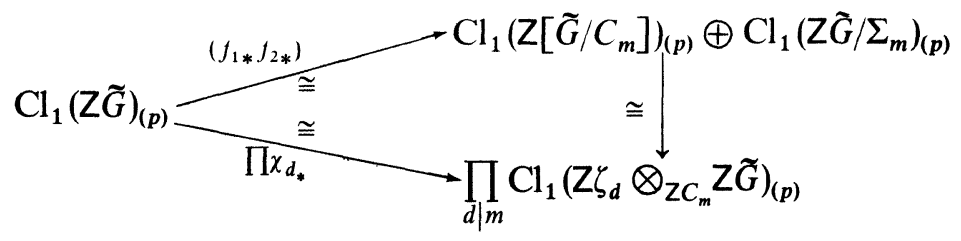

where each $\chi_{d_{*}}$ is induced by some character $\chi_{d}: C_{m} \rightarrow\left\langle\zeta_{d}\right\rangle$. In particular, $f_{1 *}=\chi_{1 *}$. All of these maps are induced by inclusions of orders in $Q \tilde{G}$ of index prime to $p\left(Z C_{m}\right.$ has index prime to $p$ in its maximal order, by [24, Theorem 41.1]), and are therefore all isomorphisms by [20, Proposition 1.2].

By naturality, $f_{1 \dot{*}^{\circ}} \beta_{(p)}^{\#}=\left(\beta / C_{m}\right)_{z}^{\#}$. To see that $f_{2 *} \circ \beta_{(p)}^{\#}=1$, we must show that $\chi_{d_{*}}{ }^{\circ} \beta_{(p)}^{\#}=1$ for all $1 \neq d \mid m$. It suffices to do this when $d$ $=m$ (otherwise, $G$ can be replaced by the appropriate quotient). We may also assume that $G$ is $p$-hyperelementary - since $K_{1}(Z G)_{(p)}$ is generated by $p$-hyperelementary induction otherwise.

STEP 1. Write $\beta^{\#}=\beta_{(p)}^{\#}$ for short. Note first that

$$
\begin{aligned}
&\left(Z \zeta_{m} \otimes_{Z C_{m}} \mathrm{Z} \tilde{G}\right) /\left(1-\chi_{m}(z)\right) \cong Z \zeta_{m} /\left(1-\zeta_{m}\right) \otimes_{Z} Z G \\
& \cong 0 \text { if } m \text { is not a prime power } \\
& \cong Z / q[G] \text { if } m=q^{l}, q \text { some prime }
\end{aligned}
$$

(see [31, Propositions 7-6-2 and 7-4-1]). In particular, by Theorem 1.1 (iii), $\chi_{m *} \circ \beta_{z}^{\#}=1$ if $m$ is not a prime power.

Assume now that $m=q^{l}$ for some prime $q$. Then, by Theorem 1.1 again, the following square commutes,

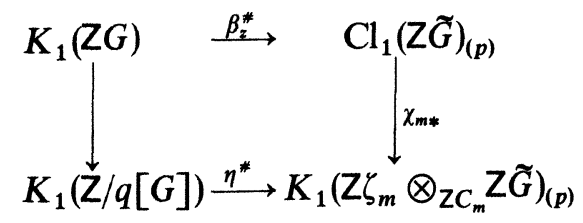


where $\eta=1-\chi_{m}(z)$. Let $G_{0} \subseteq G$ be a subgroup of $q$ power index and of order prime to $q$ ( $G$ is $p$-hyperelementary and $q \neq p)$. Then the inclusion is split by some $\alpha: G \rightarrow G_{0}$, and

(2)

$$
K_{1}(Z G)=\operatorname{Im}\left[K_{1}\left(Z G_{0}\right) \rightarrow K_{1}(Z G)\right] \oplus \operatorname{Ker}\left[\alpha_{*}: K_{1}(Z G) \rightarrow K_{1}\left(Z G_{0}\right)\right] .
$$

Since $\operatorname{Ker}(\alpha)$ is a $q$-group,

$$
\operatorname{Ker}\left[K_{1}(\mathrm{Z} / q[G]) \rightarrow K_{1}\left(\mathrm{Z} / q\left[G_{0}\right]\right)\right]
$$

is a $q$-group $\left(\operatorname{Ker}\left[\mathrm{Z} / q[G] \rightarrow Z / q\left[G_{0}\right]\right]\right.$ is nilpotent by $[10$, Proposition 5.26]). So by (1) above,

$$
\chi_{m *} \circ \beta^{\#} \mid \operatorname{Ker}\left(\alpha_{*}\right)=1 .
$$

Thus, by (2), we are reduced to considering the case where $G_{0}=G$, i.e., where $q \nmid|G|$ (and $m=q^{l}$ ). Then we can write $\widetilde{G}=C_{m} \times G^{\prime}$, and $\beta \mid G^{\prime}$ is onto with $p$-group kernel. In particular,

$$
\mathrm{Z} \zeta_{m} \otimes_{Z C_{m}} \mathrm{Z} \tilde{G}=\mathrm{Z} \zeta_{m}\left[G^{\prime}\right]
$$

STEP 2. Write $\chi_{m}(z)=\zeta x$, where $x$ generates $\operatorname{Ker}\left(\beta \mid G^{\prime}\right)$ and $\zeta$ is a primitive $m$ th root of unity. Fix $M \in \mathrm{GL}_{n}(Z G)$, let $A, B \in M_{n}\left(Z G^{\prime}\right)$ be liftings of $M$ and $M^{-1}$, respectively, and choose $X \in M_{n}\left(Z \zeta_{m}\left[G^{\prime}\right]\right)$ such that $A B-(1-\zeta x) X=I$. Then, by definition,

$$
\chi_{m *} \circ \beta_{z}^{\#}([M])=\left[\begin{array}{cc}
A & X \\
1-\zeta x & B
\end{array}\right] \in \mathrm{Cl}_{1}\left(Z \zeta_{m}\left[G^{\prime}\right]\right) .
$$

Since $\operatorname{Ker}\left(\beta \mid G^{\prime}\right)$ is a $p$-group, $\operatorname{Ker}\left[\hat{Z}_{p}\left[G^{\prime}\right] \rightarrow \hat{Z}_{p}[G]\right]$ is nilpotent $\bmod p[10$, Proposition 5.26]. It follows that $A$ is invertible in $M_{n}\left(\hat{Z}_{p}\left[G^{\prime}\right]\right)$. Write

$$
\mathrm{Z}[1 / p] \zeta_{m}\left[G^{\prime}\right]=\mathrm{Z}[1 / p] \zeta_{m}[G] \times \mathfrak{A} ; \mathrm{Q} \zeta_{m}\left[G^{\prime}\right]=\mathrm{Q} \zeta_{m}[G] \times R .
$$

Then $A \in \mathrm{GL}_{n}\left(\mathrm{Z}[1 / p] \zeta_{m}[G]\right)$ and $1-\zeta x \in \mathfrak{U}^{*}$ (for each simple component of $R, \zeta x$ goes to a primitive $m p^{i}$ th root of 1 with $i \geqq 1$ ). By Lemma 2.5 (and Theorem 2.1 (iii)),

(3) $\chi_{m *} \circ \beta_{z}^{*}([M])=\left[\begin{array}{cc}A & X \\ 1-\zeta x & B\end{array}\right]=\partial(1,\{1-\zeta x,[A]\}) \in \mathrm{Cl}_{1}\left(\mathrm{Z} \zeta_{m}\left[G^{\prime}\right]\right)$,

where $\partial: C_{p}\left(\mathrm{Q} \zeta_{m}\left[G^{\prime}\right]\right) \rightarrow \mathrm{Cl}_{1}\left(\mathrm{Z} \zeta_{n}\left[G^{\prime}\right]\right)$ is the boundary map of Theorem 2.1, $[A] \in K_{1}\left(\hat{Z}_{p}\left[G^{\prime}\right]\right)$, and

$$
(1,\{1-\zeta x,[A]\}) \in C_{p}\left(\mathrm{Z} \zeta_{m}[G]\right) \times C_{p}(R)=C_{p}\left(\mathrm{Z} \zeta_{m}\left[G^{\prime}\right]\right)
$$


STEP 3. Fix an element $a \in C_{m}$ of order $q\left(m=q^{l}\right)$. Using Theorem 4.1, choose some

$$
\tilde{u} \in K_{1}\left(\hat{Z}_{p} \tilde{G}\right)=K_{1}\left(\hat{Z}_{p}\left[C_{m} \times G^{\prime}\right]\right): \quad \Gamma_{\tilde{G}}(\tilde{u})=\frac{-1}{q^{l-1}} a \Gamma_{G^{\prime}}([A]) .
$$

Write $\mathrm{Gal}=\mathrm{Gal}\left(\mathrm{Q} \zeta_{m} / \mathrm{Q}\right)$, and regard it as a group of automorphisms of $\hat{Z}_{p}\left[C_{m} \times G^{\prime}\right]$, as well as $\hat{Z}_{p} \zeta_{m}\left[G^{\prime}\right]$. Then

$$
\begin{aligned}
\Gamma_{\widetilde{G}}\left(\sum_{\eta \in \mathrm{Gal}} \eta_{*}(\tilde{u})\right) & =-\left(a+a^{2}+\ldots+a^{q-1}\right) \Gamma_{G^{\prime}}([A]) \\
& =\Gamma_{G^{\prime}}([A])-\left(1+a+\ldots+a^{q-1}\right) \Gamma_{G^{\prime}}([A]) .
\end{aligned}
$$

Note that $\Phi\left(1+a+\ldots+a^{q-1}\right)=\left(1+a+\ldots a^{q-1}\right)\left(\Phi\left(a^{i}\right)=a^{p i}\right)$; so that

$$
\log \left(\prod_{\eta \in \mathrm{Gal}} \eta_{*}(u)\right) \equiv \log ([A])\left(\dot{\bmod }\left(1+a \ldots+a^{p-1}\right) \hat{\mathrm{Q}}_{p} \tilde{G}\right) .
$$

It follows that

$$
\log \left(\prod_{\eta \in \mathrm{Gal}} \eta\left(\chi_{m *}(\tilde{u})\right)\right)=\log ([A]) \in H_{0}\left(\tilde{G} ; \hat{\mathrm{Q}}_{p} \zeta_{m}\left[G^{\prime}\right]\right) .
$$

So by Theorem 2.3 and (3),

$$
\bar{\chi}_{m *} \circ \beta^{\#}([M])=\partial(1,\{1-\zeta x,[A]\})=\prod_{\eta \in G a l} \partial\left(1,\left\{1-\zeta x, \eta\left(\chi_{m *}(\tilde{u})\right)\right\}\right) .
$$

Since $C_{p}\left(Q \zeta_{m}\left[G^{\prime}\right]\right)$ depends only on $p$-power roots of unity, any $\eta \in \mathrm{Gal}$ acts trivially on it. Hence,

$$
\begin{aligned}
\chi_{m *} \circ \beta^{*}([M]) & =\prod_{\eta \in \mathrm{Gal}} \partial\left(1,\left\{1-\eta(\zeta) x, \chi_{m *}(\tilde{u})\right\}\right) \\
& =\partial\left(1,\left\{\prod_{\substack{i=1 \\
q \chi i}}^{q^{l}}\left(1-\zeta^{i} x\right), \chi_{m *}(\tilde{u})\right\}\right) \quad\left(m=q^{l}=|\zeta|\right) \\
& =\partial\left(1,\left\{\left(1-x^{q^{l}}\right) /\left(1-x^{q^{l-1}}\right), \chi_{m *}(\tilde{u})\right\}\right) \\
& =\chi_{m *} \circ \partial\left(1,\left\{\left(1-x^{\prime}\right) /\left(1-x^{\prime \prime}\right), \tilde{u}\right\}\right) \in \mathrm{Cl}_{1}\left(\mathrm{Z}_{m}\left[G^{\prime}\right]\right),
\end{aligned}
$$

where $x^{\prime}=x^{q^{l}}$ and $x^{\prime \prime}=x^{q^{l-1}}$ both generate $\operatorname{Ker}\left(\beta \mid G^{\prime}\right)$.

STEP 4. We now apply the notation of (4.3), as well as Propositions 4.4 and 4.7 , to the surjection

$$
\tilde{\beta}: \tilde{G}=C_{m} \times G^{\prime} \rightarrow C_{m} \times G .
$$

We have $\operatorname{Ker}(\widetilde{\beta})=\operatorname{Ker}\left(\beta \mid G^{\prime}\right)$ is a $p$-group, and $x, x^{\prime}, x^{\prime \prime}$ are all generators. 
Note that $\tilde{\beta}_{*}([A])=i_{*}([M])$ in the notation of $(4.3)$, where $[M] \in K_{1}(Z G) \subseteq K_{1}\left(Z\left[C_{m} \times G\right]\right)$. Hence, by Proposition 4.4,

$$
\Gamma([A]) \in X \cap H_{0}\left(G^{\prime} ; \hat{Z}_{p} G^{\prime}\right) .
$$

From the definition of $X$, it follows that

$$
\Gamma_{G}(\tilde{u})=\frac{-1}{q^{l-1}} a \Gamma_{G^{\prime}}([A]) \in X,
$$

and by Proposition 4.7 (ii):

$$
\partial\left(1,\left\{1-x^{\prime}, \tilde{u}\right\}\right)=\partial\left(1,\left\{1-x^{\prime \prime}, \tilde{u}\right\}\right) \in \mathrm{Cl}_{1}(Z \widetilde{G}) .
$$

Together with (5), this implies that $\chi_{m *}{ }^{\circ} \beta^{\#}([M])=1$, and we are done.

Note that for any surjection $\beta: \widetilde{G} \rightarrow G$ of finite groups with $\operatorname{Ker}(\beta)$ central and cyclic, Theorem 4.10 can be used to construct groups $W_{\beta} \supseteq W_{\beta}^{\prime}$ such that $\beta^{*}$ factors as a composite

$$
\beta^{*}: K_{1}(\mathrm{ZG}) \rightarrow W_{\beta} / W_{\beta}^{\prime} \rightarrow \mathrm{Cl}_{1}(\mathrm{Z} \tilde{G})
$$

with properties analogous to those in Theorem 4.9. In practice, however, it seems simplest just to use Theorems 4.9 and 4.10 directly.

We end by listing some consequences of Theorems 4.9 and 4.10 .

THEOREM 4.11. Let $\beta: \widetilde{G} \rightarrow G$ be any surjection of finite groups such that $\operatorname{Ker}(\beta)$ is cyclic and central in $\mathcal{G}$. Then

(i) $\beta^{*}=\beta_{z}^{*}: K_{1}(\mathrm{ZG}) \rightarrow K_{1}(\mathrm{Z} \widetilde{G})$ is independent of the choice of generator $z \in \operatorname{Ker}(\beta)$.

(ii) $\exp \left(\operatorname{Im}\left(\beta^{*}\right)\right)|| \operatorname{Ker}(\beta) \cap[\widetilde{G}, \widetilde{G}] \mid$.

(iii) $\operatorname{Im}\left(\beta^{*}\right)$ can have $p$-torsion only for primes $p$ where $G_{p}$ is non-abelian.

(iv) $\beta^{*}(\mathrm{Wh}(G))=\beta^{\#}\left(S K_{1}(\mathrm{Z} G)\right)$ if $G_{2} \triangleleft G$.

(v) Fix $p|| \operatorname{Ker}(\beta) \mid$, let $K \subseteq \operatorname{Ker}(\beta)$ be the subgroup of torsion prime to $p$, and let $\beta / K: \widetilde{G} / K \rightarrow G$ be the reduction of $\beta$. Then

$$
\operatorname{Im}\left(\beta^{\#}\right)_{(p)} \cong \operatorname{Im}\left((\beta / K)^{*}\right) .
$$

Proof. Point (v) is immediate from Theorem 4.10. Also by Theorem 4.10 , it suffices to prove (i) to (iv), when $\operatorname{Ker}(\beta)$ is a $p$-group (and $\operatorname{Im}\left(\beta_{z}^{*}\right)$ is $p$-power torsion). Then $\beta_{z}^{*}$ is independent of $z$ by Theorem 4.9 (ii); and by Theorem 4.9 (i):

$$
\exp \left(\operatorname{Im}\left(\beta^{*}\right)\right)\left|\exp \left(W_{\beta}\right)\right||\operatorname{Ker}(\beta) \cap[G, G]| .
$$

To prove (iii) and (iv), it also suffices to consider the case where $G$ (and 
hence $\tilde{G})$ are $p$-hyperelementary. Write $\tilde{G}=C_{m} \rtimes \tilde{\pi}, G=C_{m} \rtimes \pi$, where $p \nmid m$ and $\tilde{\pi}, \pi$ are $p$-groups (so $\widetilde{G}_{r}=C_{m}$ ). If $\tilde{\pi}$ is abelian, then

$$
\begin{aligned}
W_{\beta, h} & =\operatorname{Ker}(\beta) \cap[\tilde{\pi}, C(h) \cap \tilde{\pi}] \\
& =\langle[n, g] \in \operatorname{Ker}(\beta): n \in \tilde{\pi}, h \in C(h) \cap \tilde{\pi}\rangle \subseteq W_{\beta, h}^{\prime}
\end{aligned}
$$

for any $h \in C_{m}$; thus $W_{\beta}=W_{\beta}^{\prime}$, and $\beta^{\#}=1$.

If $p$ is odd, then (iv) follows from Proposition 1.3. If $p=2$ and $G_{2} \triangleleft G$, then $G$ is $p$-elementary, $C(h)=\tilde{G}$ for any $h \in \widetilde{G}_{r}=C_{m}$; and so $\Omega_{\beta} \mid S K_{1}(Z G)$ is onto by Theorem 4.9 (iii). It follows that

$$
\beta^{\#}\left(S K_{1}(\mathrm{Z} G)\right)=\Theta_{\beta} \cdot \Omega_{\beta}\left(S K_{1}(\mathrm{Z} G)\right)=\operatorname{Im}\left(\Theta_{\beta}\right)=\operatorname{Im}\left(\beta^{\#}\right) .
$$

\section{REFERENCES}

1. R. Alperin, K. Dennis, R. Oliver, and M. Stein, $S K_{1}$ of finite abelian groups: II, to appear.

2. D. Anderson, The Whitehead torsion of the total space of a fiber bundle, Topology 11 (1972), 179-194.

3. E. Artin and H. Hasse, Die beiden Ergänzungssätze zum Reziprozitätsgesetz der $l^{n}$-ten Potenzreste im Körper der $l^{n}$-ten Einheitswurzeln, Abh. Math. Sem. Univ. Hamburg 6 (1928), 146-162.

4. A. Bak, The involution on Whitehead torsion, General Topology Appl. 7 (1977), 201-206.

5. A. Bak, K-theory of forms (Ann. of Math. Studies 98) Princeton University Press, Princeton, N. J., 1981.

6. A. Bak, $A$ norm theorem for $\mathrm{K}_{2}$ of global fields, in Algebraic Topology (Proc. Aarhus, 1982) eds. I. Madsen and B. Oliver (Lecture Notes in Math. 1051) pp. 1-7, Springer-Verlag, Berlin - Heidelberg - New York, 1984.

7. A. Bak and U. Rehmann, The congruence subgroup and metaplectic problems for $\mathrm{SL}_{n \geqq 2}$ of division algebras, J. Algebra 78 (1982), 475-547.

8. H. Bass, Algebraic K-theory, W. A. Benjamin, New York, Amsterdam, 1968.

9. H. Bass, J. Milnor, and J.-P. Serre, Solution of the congruence subgroup problem for $\mathrm{SL}_{n}(n$ $\geqq 3$ ) and $\operatorname{Sp}_{2 n}(n \geqq 2)$, Inst. Hautes Études Sci. Publ. Math. 33 (1967), 59-137.

10. C. Curtis and I. Reiner, Methods of representation theory, I: With applications to finite groups and orders, J. Wiley and Sons, New York, 1981.

11. D. Grayson, Higher algebraic K-theory II, in Algebraic K-theory (Proc. Evanston, 1976) ed. M. R. Stein, (Lecture Notes in Math. 551), pp. 217-240, Springer-Verlag, Berlin Heidelberg - New York, 1976.

12. M. Keating, Values of tame symbols on division algebras, J. London Math. Soc. 14 (1976), 25-30.

13. K. Kwun and R. Szczarba, Product and sum theorems for Whitehead torsion, Ann. of Math. 82 (1965), 183-190.

14. T.-Y. Lam, Induction theorems for Grothendieck groups and Whitehead groups of finite groups, Ann. Sci. École Norm. Sup. 1 (1968), 91-148.

15. J. Milnor, Introduction to algebraic K-theory, (Ann. of Math. Studies 72) Princeton University Press, Princeton, N. J., 1972. 
16. H. J. Munkholm and E. K. Pedersen, Whitehead transfers for $S^{1}$-bundles, an algebraic description, Comment. Math. Helv. 56 (1981), 404-430.

17. R. Oliver, $S K_{1}$ for finite group rings: I. Invent. Math. 57 (1980), 183-204.

18. R. Oliver, $S K_{1}$ for finite group rings: II. Math. Scand. 47 (1980), 195-231.

19. R. Oliver, $S K_{1}$ for finite group rings: III in Algebraic K-theory (Proc. Evanston, 1980) eds. E. M. Friedlander, M. R. Stein (Lecture Notes in Math. 854), pp. 229-337, SpringerVerlag, Berlin - Heidelberg - New York, 1981.

20. R. Oliver, $S K_{1}$ for finite group rings: IV, Proc. London Math. Soc. 46 (1983), 1-37.

21. R. Oliver, $S K_{1}$ for finite group rings: $\mathrm{V}$, to appear.

22. R. Oliver, Logarithmic descriptions of $K_{1}^{\prime}\left(\hat{Z}_{p} G\right)$ and class groups of symmetric groups, Math. Ann., to appear.

23. D. Quillen, High'r algchraic K-theory I in Algebraic K-theory I. Higher $K$-theories (Proc. Seattle, 1972) ed. H. Bass (Lecture Notes in Math. 341), pp. 85-147, Springer-Verlag, Berlin - Heidelberg - New York, 1973.

24. I. Reiner, Maximal orders, (London Math. Soc. Monographs 5) Academic Press, London, New York, San Francisco, 1975.

25. P. Roquette, Realisierung von Darstellungen endlicher nilpotenter Gruppen, Ark. Math. 9 (1958), 241-250.

26. J.-P. Serre, Corps Locaux (Publ. Inst. Math. Univ. Nancago 8), Hermann, Paris, 1968.

27. U.Stammbach, Homology in group theory (Lecture Notes in Math. 359), Springer-Verlag, Berlin - Heidelberg - New York, 1973.

28. R. Swan, Excision in algebraic K-theory, J. Pure Appl. Algebra 1 (1971), 221-252.

29. C. T. C. Wall, Surgery on compact manifolds (London Math. Soc. Monographs 1) Academic Press, London, New York, 1970.

30. C. T. C. Wall, Norms of units in group rings, Proc. London Math. Soc. 29 (1974), 593-632.

31. E. Weiss, Algebraic number theory, McGraw-Hill Book Co., Inc., New York, San Francisco, Toronto, London, 1963.

MATEMATISK INSTITUT

AARHUS UNIVERSITET

NY MUNKEGADE 116

DK-8000 AARHUS C

DENMARK 